Florida International University

FIU Digital Commons

FIU Electronic Theses and Dissertations

University Graduate School

6-26-2017

\title{
The Effects of Children's Spiritual Coping after Parent, Grandparent or Sibling Death on Children's Grief, Personal Growth, and Mental Health
}

Ivette M. Hidalgo

Florida International University, ihida001@fiu.edu

DOI: $10.25148 /$ etd.FIDC001930

Follow this and additional works at: https:// digitalcommons.fiu.edu/etd

Part of the Nursing Commons, Other Religion Commons, and the Psychiatry and Psychology Commons

\section{Recommended Citation}

Hidalgo, Ivette M., "The Effects of Children's Spiritual Coping after Parent, Grandparent or Sibling Death on Children's Grief, Personal Growth, and Mental Health" (2017). FIU Electronic Theses and Dissertations. 3467.

https://digitalcommons.fiu.edu/etd/3467

This work is brought to you for free and open access by the University Graduate School at FIU Digital Commons. It has been accepted for inclusion in FIU Electronic Theses and Dissertations by an authorized administrator of FIU Digital Commons. For more information, please contact dcc@fiu.edu. 


\section{FLORIDA INTERNATIONAL UNIVERSITY \\ Miami, Florida}

\section{THE EFFECTS OF CHILDREN'S SPIRITUAL COPING AFTER PARENT, GRANDPARENT OR SIBLING DEATH ON CHILDREN'S GRIEF, PERSONAL GROWTH, AND MENTAL HEALTH}

A dissertation submitted in partial fulfillment of the requirements for the degree of DOCTOR OF PHILOSOPHY

in

NURSING

by

Ivette M. Hidalgo 
To: Dean Ora Lea Strickland

College of Nursing and Health Sciences

This dissertation, written by Ivette M. Hidalgo, and entitled, The Effects of Children's Spiritual Coping After Parent, Grandparent or Sibling Death on Children's Grief, Personal Growth and Mental Health, having been approved in respect to style and intellectual content, is referred to you for judgment.

We have read this dissertation and recommend that it be approved.

Dorothy Brooten

Jean Hannan

Whitney Bauman

JoAnne Youngblut, Major Professor

Date of Defense: June 26, 2017

The dissertation of Ivette M. Hidalgo is approved.

Dean Ora Lea Strickland
College of Nursing and Health Sciences

\begin{abstract}
Dean Andres G. Gil
Vice President for Research and Economic Development and Dean of the University Graduate School
\end{abstract}

Florida International University, 2017 


\section{DEDICATION}

I dedicate this dissertation to my family. To my husband, thank you for always being there for me every step of the way and for always believing in me. For that, I love you today and always. To my daughter Isabella, my little angel, thank you for inspiring me to reach for the stars. To my parents, thank you for instilling in me a passion for learning. You have both been candid examples of how through one's effort, honesty, and perseverance one can triumph. I also dedicate this dissertation to my dear friend Dr. Raquel Marrero, thank you for being my guiding light in all sense of the word. You have shown me the power of faith and prayer and, most of all, the importance of love for mankind. I hope that through my work I can help others in the same manner in which you have helped me. 


\section{ACKNOWLEDGMENTS}

I especially want to recognize Dr. JoAnne Youngblut, Chair of my committee. She has taught me the values of patience, perseverance, and the ability to keep focused and goal oriented despite any obstacles in my path. I want to express my gratitude to Dr. JoAnne Youngblut. Her knowledge of statistics and her exceptional editorial skills made it possible for this dissertation to be completed. It has been a memorable journey.

I also want to thank my committee members, Dr. Dorothy Brooten, Dr. Jean Hannan, and Dr. Whitney Bauman for their time, dedication, and knowledge. To my beloved sister Rosa, I thank you for taking the time to listen. To my sweet friends Rosa Roche, Bela Florentin, and Laura Hernandez I thank you for always being there for me throughout all these years and for your kind words of encouragement and advice. My deepest appreciation goes to Chaplain Shirley Brown, Mary Kennerk, Dr. Rosalyn Berry, Joanna Commendeiro, Janet Bell-Taylor, and Jackie Gonzalez for their assistance with the recruitment of families for my study.

I would like to thank all of the children that participated in this study as well as

their families. Finally, and most importantly, I want to thank God for walking beside me and at times carrying me throughout this journey. 


\section{ABSTRACT OF THE DISSERTATION \\ THE EFFECTS OF CHILDREN'S SPIRITUAL COPING AFTER PARENT, GRANDPARENT OR SIBLING DEATH ON CHILDREN'S GRIEF, PERSONAL GROWTH, AND MENTAL HEALTH}

by

Ivette M. Hidalgo

Florida International University, 2017

Miami, Florida

Professor JoAnne Youngblut, Major Professor

Parental death can have a negative impact on children's lives with changes in economic resources, and lead to change in residence, loss of contact with friends and neighbors, change in caretakers, and loss of time with the surviving parent. Research on the effects of a grandparent's death on children is limited. Death of a sibling signifies the loss of a role model, friend, confidante, and playmate. The purpose of this correlational study was to identify: 1) differences in the spiritual coping strategies used by children across age groups, gender, race/ethnicity, participation in religious rituals and practices, and the relationship of the deceased to the child (parent, grandparent or sibling), and 2) the relationship between children's use of spiritual coping strategies and grief, personal growth, anxiety, and depression after the death, with and without controls for child's age, gender, race/ethnicity, participation in religious rituals and practices, and relationship of the deceased to the child. A sample of 97 children, 8 to 18 years old and $64 \%$ Hispanic completed the Spiritual Coping Strategies Scale, Hogan Inventory of Bereavement, Spence Children's Anxiety Scale, and Child Depression Inventory, and their parents 
completed a demographic form. Children who participated in religious rituals after the death used less religious coping strategies than children who did not participate. When child's age, gender, race/ethnicity, participation in religious rituals and practices, and relationship of the deceased were controlled, greater use of spiritual coping, but not religious coping, and greater grief were significantly related to greater personal growth and greater anxiety. Younger children and Black children had significantly greater anxiety. Only grief was significantly related to depression. Children who experienced the death of a parent, grandparent, or sibling had similar outcomes. The results of this pioneer study will add knowledge to the state of the science regarding the effects of children's spiritual coping after parent, grandparent, or sibling death on their grief, personal growth, and mental health which is a subject area were very little is known. 


\section{TABLE OF CONTENTS}

CHAPTER $\quad$ PAGE

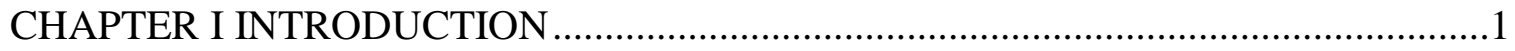

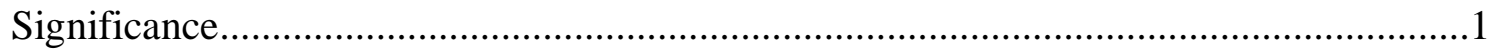

Children's Responses to Parent, Grandparent, and Sibling death .....................................

Interventions Tested for Children ................................................................................5

Children's Religion and Spirituality ..........................................................................

Cultural Differences in Death Rituals for Deceased Children and Adults ......................12

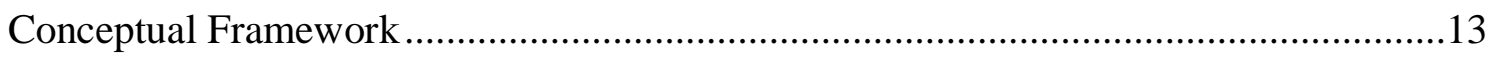

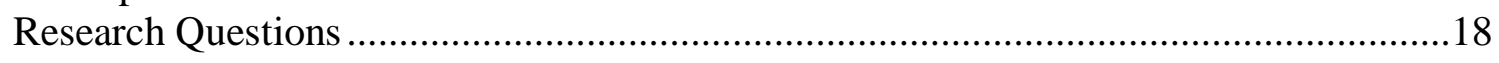

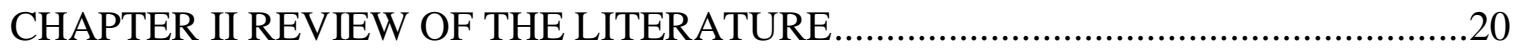

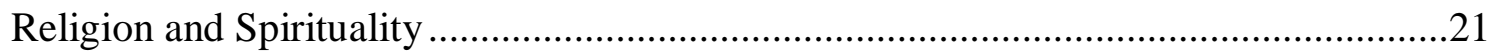

Children's Development of Understanding about Religion and Religiosity ...................21



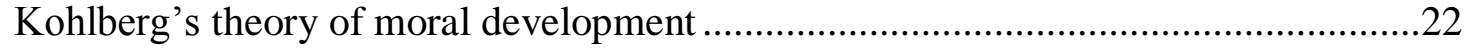

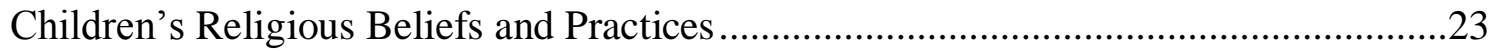

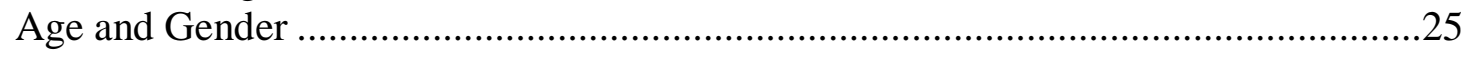



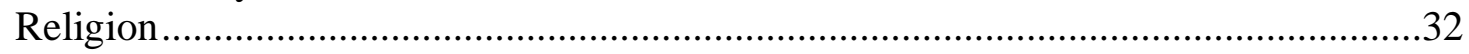

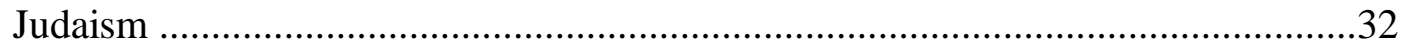

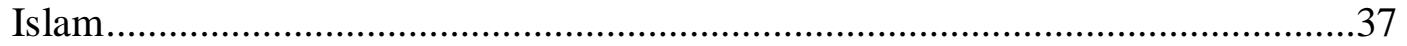

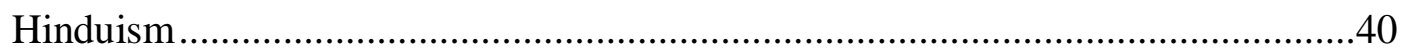

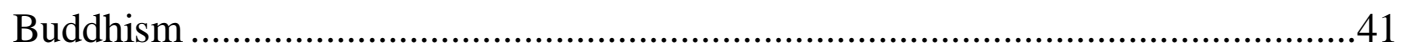

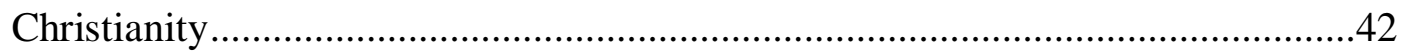

Changes in Religious Beliefs and Practices after the Death of a Sibling ........................44

Understanding of Death and Death Rituals .................................................................45

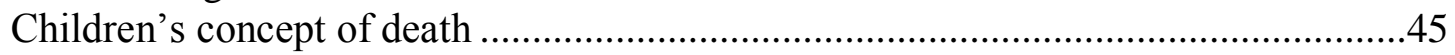

Influence of religion on children's concept of death ...........................................4

Experience with Death and Death Rituals ..................................................................49

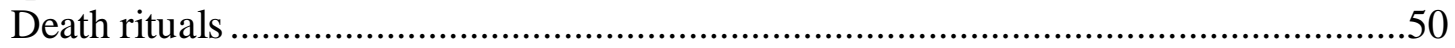

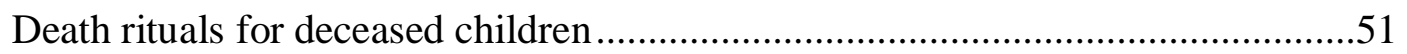

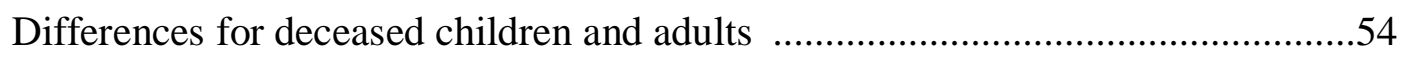

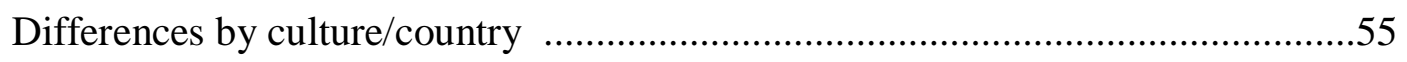

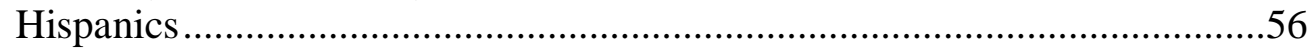

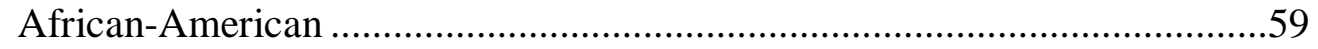





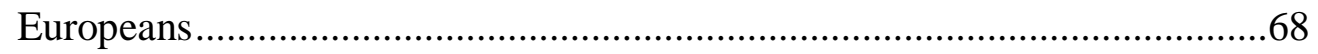



Response to Death of a Parent, Grandparent, or Sibling ............................................73

Conceptual definitions of grief and bereavement ……….....................................73 
Behavioral manifestations of children's grief after the death of a parent,

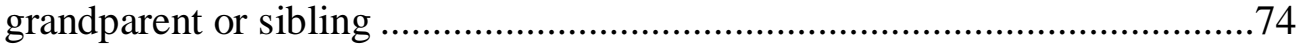

Conceptual definition of grief disorder ....................................................96

Prolonged grief/complicated grief disorder, post-traumatic stress disorder and



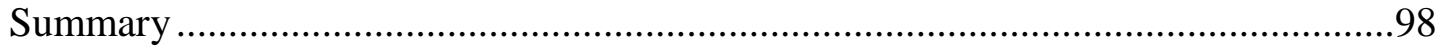

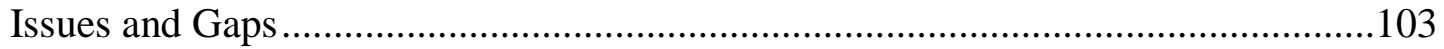

CHAPTER III DESIGN AND METHODS ............................................................ 106

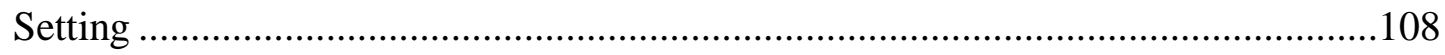

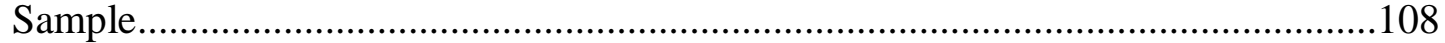

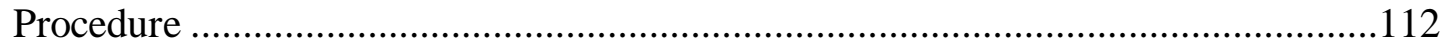



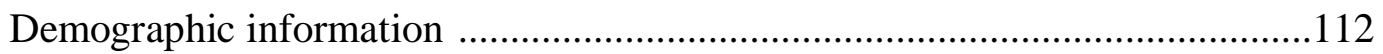

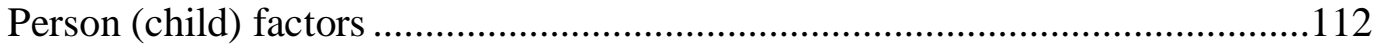

Participation in religious rituals and practices ......................................112

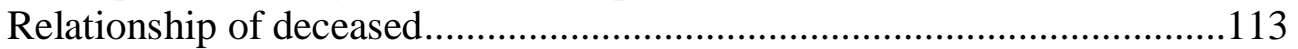



Children's grief and personal growth .......................................................113

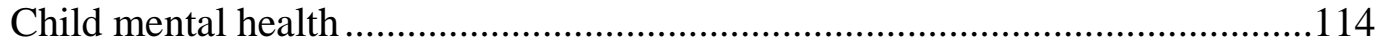



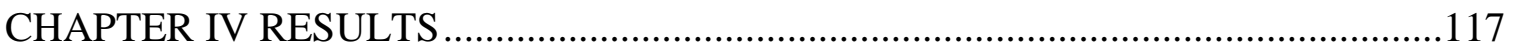

Description of the sample .................................................................... 117

Differences in spiritual coping by person factors ….....................................118

Spiritual coping strategies, grief, and outcomes ........................................121

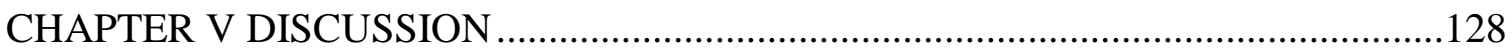

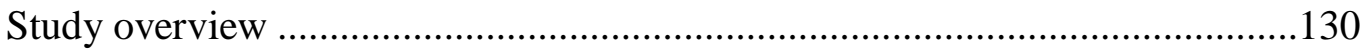

Differences in spiritual coping by person factors ........................................131

Spiritual coping strategies, grief, and outcomes ..........................................133

Relationships with control for person factors .............................................136

Effects of spiritual coping strategies and grief on outcomes with control for

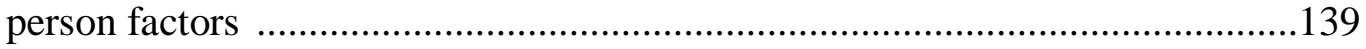

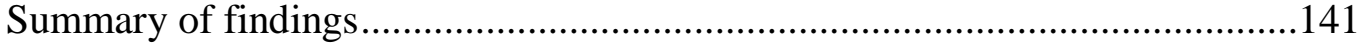

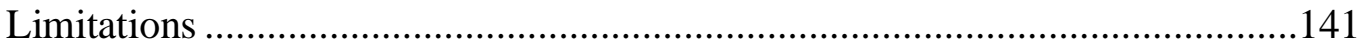

Implications for practice and education .................................................. 142

Implications for research.................................................................. 143





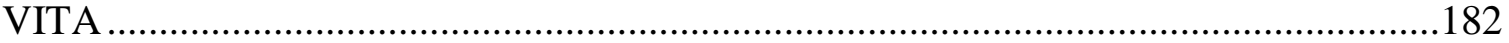




\section{LIST OF TABLES}

TABLE

PAGE

Table 1 Sample Demographics..............................................119

Table 2 Differences in Spiritual Coping by Person Factors....................... 120

Table 3 Correlations between Spiritual Coping Strategies and Grief, Personal Growth, Anxiety, and Depression..................................................122

Table 4 Correlations among independent variables.................................. 124

Table 5 Multiple regression analyses for grief, personal growth, anxiety, and

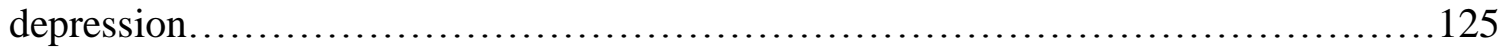

Table 6 Multiple regression analyses for the outcomes of personal growth, anxiety,

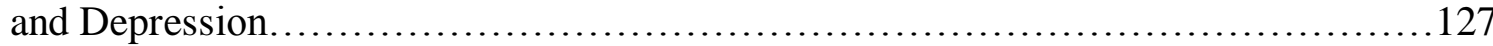




\section{CHAPTER I}

\section{INTRODUCTION}

\section{Significance}

In 2016, the Centers for Disease Control and Prevention (CDC) determined that in 2014 the United States had a total of 23,215 deaths that occurred in infants under age one year. The annual death rate among children was 3,830 per 100,000 children 1 to 4 years); 5,250 per 100,000 children 5 to 14 years; and 9,586 per 100,000 adolescents 15 to 19 years (CDC, 2016). This large number of child deaths each year also affects a larger number of siblings (Fletcher, Mailick, Song, \& Wolfe, 2013). This supports the need for research on children's responses to sibling death. In 2014 there were a total of 2,626,418 adult deaths in the US (CDC, 2016). Therefore, many children may experience death of a parent or grandparent. The majority of the research focuses on the effects that children's deaths have on parents, with little attention to the emotional, psychological, and behavioral grieving responses that a parent's, grandparent's, or sibling's death during childhood has on the surviving children. The literature often refers to surviving children as "forgotten grievers" since their loss is insufficiently acknowledged (Crehan, 2004; Horsley \& Patterson, 2006).

The death of a sibling signifies the loss of a role model, friend, confidante, and playmate (Woodgate, 2006). Research in sibling bereavement has found an array of grieving responses (emotional, behavioral, social, physical) and decline in school performance (Fanos, Little, \& Edwards, 2009). Bereaved siblings are at risk for developing mental health disorders, such as depression, prolonged grief disorder (PGD), and post-traumatic stress disorder (PTSD) (Barrera, Alam, D’Agostino, Nicholas, \& 
Schneiderman, 2013; Martinson, \& Campos, 1991). Parental death can have a negative impact on children's lives. Through changes in economic resources and residence, loss of contact with friends and neighbors, change in caretakers, and diminished time with the surviving parent (Wolchik, Tein, Sandler, \& Ayers, 2006). Parentally bereaved children not only face the permanent physical absence of their loved one but also may have been exposed to traumatic images and situations associated with the end of the parent's life, thereby increasing the likelihood of developing PTSD (Kaplow, Layne, \& Pynoos, 2014). Research on the effects that the death of a grandparent has on the child is limited. Research has found that religious and spiritual forms of coping are important in promoting positive posttraumatic growth in children (Bryant-Davis et al., 2012).

Children express their spiritual needs in ways consistent with their age, religious background, culture, and developmental level (Farrell et al., 2008). Fowler (1981) suggests that children's understanding of faith occurs in stages consistent with Erikson's theory of psychosocial development, Piaget's theory of cognitive development and Kohlberg's theory of moral development. Children as young as 4 to 6 years viewed the mind and an agent (God) as necessary for prayer (Bamford \& Lagattuta, 2010). By age 8, children associate praying with negative emotions and feeling better after praying. It is imperative to identify how children's descriptions of their religious beliefs and practices differ with the child's age, gender, race/ethnicity, and religion. The purpose of this correlational study was to identify 1) differences in the spiritual coping strategies used by children across age groups, gender, race/ethnicity, participation in religious rituals and practices, and the relationship of the deceased to the child (parent, grandparent or sibling), and 2) the relationship between children's spiritual coping strategies and grief, 
personal growth, anxiety, and depression after the death with and without controls for child's age, gender, race/ethnicity, participation in religious rituals and practices, and relationship to the deceased.

\section{Children's Responses to Parent, Grandparent, and Sibling Death}

Children's bereavement experiences may be affected by their understanding of the concept of death, and children's religion may have a direct impact on their understanding (Bering et al., 2005). Children's developmental stage when their parent, grandparent or sibling dies also might affect the grief process and may pose additional challenges for the surviving child (Lohan \& Murphy, 2001). For example, the developmentally-based psychosocial, physiological, and cognitive changes in adolescence may make grieving the death of a loved one even more difficult.

After the death of a parent, events such as being teased by children because of the parent's death, having fewer opportunities to engage in extracurricular activities, being told to act differently than they did before the death, and changes in the dynamics of the relationship between children and their surviving parent, such as receiving less attention for positive behaviors, may threaten a child's sense of self-worth (Wolchik et al., 2006). In a study by Haine, Ayers, Sandler, Wolchik, and Weyer (2003), self-esteem was found to mediate the relationship between post-death stressors and internalizing and externalizing problems.

Parents of bereaved children may not provide adequate emotional support as they try to cope with the loss themselves. Some children may not seek support or talk about their reactions, thinking that they have to protect their grieving parents (Dyregrov \& 
Dyregrov, 2005) which prevents them from expressing their own emotions and grieving the loss (Forward \& Gallie, 2003).

In a study by Servaty-Seib and Pistole (2006) when emotional closeness to the deceased person was taken into account, grandchildren who had a close relationship with their grandparent experienced greater grief than children who did not, with grief levels that resembled those from other types of loss. After an extensive review of the literature, research focused on the effects of a grandparent's death on children was non-existent.

Children manifest their grief differently from adults. Children's responses to death varied with their age due to the impact of development on children's expressions of grief (Christ, 2000). Data on childhood bereavement after sibling death are generally obtained by parent report (Paris, Carter, Day, \& Armsworth, 2009). Parents are distraught and immersed in their own grief and may not fully appreciate their children's grieving responses. It is imperative to gain a better understanding of children's grief after the sibling's death since they may not share their feelings with their parents. The children have to cope with the death of their brother or sister and the reactions of the grieving parents who are preoccupied with the sibling's death and may be emotionally absent and inattentive to the surviving child's needs (Rosen, 1986; Willis, 2002). It is important to describe and understand grief and bereavement after a sibling's death from the child's perspective.

Grief can be prolonged and can resurface throughout the child's life when they examine the death from their changing developmental perspective. Children can be deeply affected after the loss of a sibling and might experience pathological or traumatic grief. Spuij et al. (2012) found that children's prolonged grief disorder (PGD) and greater 
depression and/or posttraumatic stress disorder (PTSD) were significant predictors of their functional impairment. Complicated grief (CG) differs from depression, anxiety, and PTSD (Melhem et al., 2007). Some symptoms of CG consist of increased longing and searching for the deceased, hopelessness about the future, difficulty accepting the death, numbness and detachment from others, and anger and bitterness. Death of a sibling often leaves an immense emptiness in the hearts and lives of the surviving children.

The sibling relationship provides a socialization and developmental mechanism where they learn from each other and learn to define one another (Stormshak, Bullock, \& Falkenstein, 2009). Sometimes their relationships are characterized by friendship, companionship, and trust, and at other times they can be adversaries. Surviving children share such a strong connection or bond with the deceased sibling that their relationship can surpass death (Packman, Horsley, Davies, \& Kramer, 2006). Sometimes children keep strong bonds with the deceased sibling by wearing their clothes, playing with their toys, visiting their favorite places, eating their favorite foods, or listening to their favorite songs (Foster et al., 2011).

Children may also feel connected to their deceased brother or sister through their religious beliefs, perhaps believing they will be reunited with their brother or sister, which serves as a continuing bond (Forward \& Garlie, 2003). For many children keeping a strong bond with their deceased brother or sister helps them cope with the loss.

\section{Interventions Tested for Children}

The surviving child's developmental stage when their sibling dies affects the grief process and poses additional challenges for them (Lohan \& Murphy, 2001). Despite marked growth in bereavement research, very little is known regarding what bereaved 
children identify as helpful (Kazak \& Noll, 2004). The majority of the literature is focused on bereaved spouses and parentally bereaved children, while the death of a sibling has not received as much attention (Thompson et al., 2011). Death of a parent, grandparent, or sibling may leave the child emotionally vulnerable at a young age.

Methodological dissimilarities such as time of data collection, age of participants, and instruments used make it difficult to pinpoint specific grieving patterns in children (Barrera, Alam, Mammone D’Agostino, Nicholas, \& Schneiderman, 2013). Also, the mostly cross-sectional research (Wilkins \& Woodgate, 2005) prevents an understanding of this phenomenon over time (Holmbeck, Bruno, \& Jandasek, 2006). Data on childhood loss of a sibling are generally obtained by parent report rather than directly from the child (Paris et al., 2009).

Only a few longitudinal studies exist with regards to grief after the death of a loved one during childhood and adolescence (Wilkins \& Woodgate, 2005; Woodgate, 2006). Most of the studies pertaining to child and adolescent bereavement have concentrated on the death of a parent, not a sibling or grandparent (Sood, Razdan, Weller, \& Weller, 2006). Research with bereaved children and adults reveals that deaths which were more recent (within 1-1.5 years), viewed as unexpected, in the hospital versus home, and associated with a prolonged illness and/or suffering may be related to more severe grief reactions and distress (Barry et al., 2002; Gamino et al., 2000; Seecharan et al., 2004).

Uncomplicated bereavement in Diagnostic and Statistical Manual of Mental Disorders (DSM-4) (2000) is defined as the classic grieving process with symptoms of intense sadness, sleep and appetite problems, lack of interest in regular activities, and 
difficulty concentrating. Research in child bereavement has described an array of grieving responses: emotional (depression, sadness, anxiety, feelings of guilt), behavioral (sleep disturbances, acting out), social (withdrawal, loneliness), and deterioration in school performance (Fanos et al., 2009). Bereaved children may not find the emotional support they need from their parents since the parents are focused on their own grief and pain (DeCinque et al., 2006). Children may not confide in their parents about their grief. Some may feel they have to protect their grieving parents (Dyregrov \& Dyregrov, 2005), while others may need a break from their parent's grieving (Youngblut \& Brooten, 2013).

Oltjenbruns (2001) poses that "childhood development is ongoing; grief and feelings of loss are often repeatedly processed as children become more capable of understanding their loss" (p. 73). Children manifest their grief differently, and the duration of the grief responses cannot be conceptualized from an adult perspective. Loss in childhood can only be understood in the context of a child's cognitive, emotional, and social development (Paris et al., 2009).

Willis (2002) explains that children's unacceptable behaviors may be expressions of their confusion after the death of a loved one. Aiken (2001) indicates that children are less likely to accept death and tend to grieve intermittently for several years. Grief can be prolonged and can resurface throughout the child's life when he/she examines the death from a different perspective and developmental level.

Effective interventions educate the parents regarding children's grief reactions after the death and help them create a nurturing environment in the home where the children were able to express their emotions openly. Children did not feel that they had to keep their emotions private to protect their parents from further distress, allowing them to 
grieve and keep the lines of communication with their parents open. Childhood bereavement services facilitate children's understanding of bereavement by recognizing their feelings and helping them to integrate the death in accordance with their emotional and social development.

Children who participated in peer group activities with other bereaved children reported feeling less lonely and isolated (Cohen et al., 2006). They also felt that they could speak freely without worrying that they would hurt someone's feelings. Peer support group activities did not have much of an impact on child-parent communication about the sibling's death or on children being able to express their feelings at home. Cognitive behavioral therapy that focused on trauma and grief was found to decrease childhood traumatic grief (CTG), PTSD, anxiety, and depression scores in children over time.

Children who participated in Camp MAGIK for bereaved children agreed that the counseling sessions were the most healing part of the camp. The counseling sessions incorporated trauma interventions such as exposure, stress inoculation techniques, and cognitive structuring which have been found to decrease trauma (Cohen et al., 2006). Rituals such as the balloon release and the memorial service helped them to keep a continuing bond with the deceased, now recognized as integral to successful adjustment (Field \& Friedrichs, 2004). Continuing bonds can facilitate coping for children, comforting them after the death of the sibling. In a study by Thompson et al. (2011), parents and siblings relied on their belief in the afterlife to find comfort in their grief.

The majority of the literature focused on interventions for bereaved spouses and parentally-bereaved children, omitting sibling bereaved-children. Gender specific 
analysis was not conducted in the majority of the studies despite gender differences found in prior work on children's grief. Worden, Davies, and McCown (1999) found that adolescent girls may be more susceptible to emotional or behavioral difficulties when a sibling dies. Children may be able to cope with the death of a family member or the critical illness of a sibling by finding meaning through spirituality (Batten \& Oltjenbruns, 1999). During times of crisis and ill health, children may rely on their religion and spirituality (Bryant-Davis et al., 2012).

\section{Children's Religion and Spirituality}

The majority of the research focused on spirituality in adults, with few studies of spirituality in children (Hufton, 2006). Hay (2003) argues that all children have spirituality and are more in touch with their spirituality than adults. Children use spirituality to shape their perceptions of life (Sifers, Warren, \& Jackson, 2012). Religion can provide a fundamental meaning to human experiences and their trials and tribulations depending on the needs present at the specific time. Religious and spiritual coping strategies are used by children when they are facing stress, trauma, or illness (BryantDavis et al., 2012). An individual employs religious coping in crisis situations due to its protective factors and to gain a deeper understanding and make sense of the negative experience.

Religiousness and spirituality in children are affected by both cognitive developmental changes as Fowler's Stages of Faith theory asserts and moral developmental changes described in Kohlberg's theory of moral development (Pendleton, Cavalli, Pargament, \& Nasr, 2002). Not many researchers have focused on studying spirituality in children (Houskamp, Fisher, \& Stuber, 2004). The limited studies of 
spirituality in children are qualitative in nature and grounded in child development theory (Boynton, 2011). Consequently, the research on spirituality in children is still in its infancy. The spiritual dimension of children is poorly understood. There is research evidence for adults and adolescents that spirituality is part of their overall health, decreasing delinquency and psychopathology, and contributing to coping and resiliency (Koenig, 2004; Pendleton, et al., 2002).

Factors related to spirituality and religiosity in children and adolescents provide a foundation for healthy development that can assist children throughout their formative years to choose healthy paths and to make wise decisions (Bosacki, Elliott, Akseer, \& Bajovic, 2010). Spirituality influences how children respond to and evaluate life events and how they achieve developmental tasks. It is also associated with socialization processes, family functioning, parenting, and relationships (Houskamp et al., 2004). Data from the National Longitudinal Study of Adolescent Health demonstrated a relationship between religion in adolescents' lives and positive family relationships (Regnerus \& Burdett, 2006).

Factors related to spirituality and religiosity in children may create a basis from which to make appropriate health choices and decisions throughout their formative years that will positively affect their wellness (Bosacki et al., 2010). Spirituality and religious coping have a protective role in adolescents' lives (Gunnestead \& Thwala, 2011). Individuals who used positive religious coping methods had more positive health outcomes than people who used negative religious coping (i.e. difficult situations are God's punishment) (Pargament, 2001). 
After reviewing the literature, a common definition for spirituality was not found (Feudtner et al., 2003). Spirituality is thought to generate values and beliefs in children that influence their behaviors (Burg et al., 2011). Religion makes it possible for individuals to find meaning in situations that are difficult to understand (Bryant-Davis et al., 2012). Children's religious practices and beliefs are influenced in their daily interactions with adults and peers as well as through the media (internet and television).

Religion identity is subject to a complex pattern of influences. Adolescents who frequently attended religious services developed a more supportive social network than those who didn't (Pearce et al., 2003). Also, adolescents' psychosocial wellbeing is more strongly associated with their social experience in religious environments than with their attendance at religious services. Girls were more likely than boys to discuss issues of religiosity and spirituality (Bosacki et al., 2010).

Adults may not recognize children's spiritual cues. When children are not able to verbally express these cues due to their limited cognitive and language development (Hufton, 2006), their spiritual needs may go unnoticed. Even though young children have limited cognitive and verbal abilities to discuss concepts relating to spirituality, they can and do have deeply spiritual experiences (Wilson, 2010). Childhood is a time of wonder. Children experience the physical aspects of the world through wonder as well as its nonmaterial spiritual characteristics (Hart, 2005). A child's experience of wonder is characterized by feelings of awe, connection, and a deep sense of love. According to Hart (2005), there is a strong connection between wonder and a sense of the sacred. Instruments to measure spirituality in children do not exist (Houskamp et al., 2004); so researchers often adapt adult measures for children (Cotton et al., 2006; Fisher, 2009). 
Defining the concept of spirituality from the child's perspective is necessary for adults to understand children's spiritual needs. It is necessary to take into account the role that culture has on children's expression of religion and spirituality.

\section{Cultural Differences in Death Rituals for Deceased Children and Adults}

The US has been described as a melting pot due to its cultural diversity. Assessment of different cultures' response patterns to death requires understanding the individual's beliefs about death and their specific rituals for coping with the death (Clements et al., 2003). Each culture has distinct and specific approaches to dealing with death (Parkes, Laungani, \& Young, 1997).

Even though death is part of all humans' lives, there are pronounced cultural and religious differences in attitudes toward death (Neimeyer, Wittkowski, \& Moser, 2004). While some people view death as frightening and perplexing, others see death as a natural part of life that gives special meaning to life. These different views of death have implications for how individuals deal with fundamental existential questions such as the meaning of life and the possibility of an afterlife.

A ritual can legitimize grief, provide an arena where the death and its finality are acknowledged, and create a safe place for the mourner to express emotion. Those with common bonds, such as religion or ethnic origin, perform rituals to recognize the death and pay tribute to the deceased (Weeks, 2004). There are limited studies on the impact that death rituals have on bereaved children.

Rituals surrounding the death of a loved one have been described from a religious or cultural perspective, but with little research evidence from important informants within the culture, especially bereaved children. Also, research has examined death rituals in a 
relatively narrow framework of Christian, or at most, Christian and Jewish beliefs failing to incorporate other religions. The US is composed of individuals who practice a wide variety of religions. Failing to incorporate other religions in future research will not provide a clear picture of death rituals practiced in the US.

\section{Conceptual Framework}

This correlational study was guided by The Spiritual Framework of Coping created by Gall, Charbonneau, Clarke, Grant, Joseph, and Shouldice (2005). The Spiritual Framework of Coping is based on the Transactional Model of Stress and Coping (Lazarus \& Folkman, 1984) and incorporates its basic principles and structural components. Pargament (1997) points out that the transactional model of stress and coping serves as a starting point for understanding research focused on religiosity and spirituality. Spirituality is characterized as a complex, multidimensional construct that is apparent in an individual's beliefs, behavior, and experience (Miller \& Thoresen, 1999). The Spiritual Framework of Coping is phenomenological, dynamic and relational, and process-oriented.

Since spirituality is a multidimensional construct, it can operate at several levels of the stress and coping process at one point in time (Figure 1). It can operate at the level of primary and secondary appraisals (God attributions), spiritual person factors (beliefs), coping behavior (prayer), and coping resources (connection to nature), and meaning making (spiritual reappraisal). In this framework, spiritual person factors function as a contextual framework that makes it feasible for individuals to formulate their interpretation, comprehension, and reaction to life experiences (Krok \& Dariusz, 2008; Tuck, Alleyne, \& Thinganjana, 2006). Such beliefs may assist in creating meaning out of 
his or her suffering and creating a more hopeful attitude (Garssen, Uwland-Sikkema, \& Visser, 2015). Beliefs may also enable a more dynamic attitude toward coping and promote social support (Koenig, 1995; Levin \& Chatters, 1998) in response to stress.

Spiritual appraisals and spiritual coping behaviors function as mediating factors in the process of coping with stress. Spiritual appraisals have to do with making sense of the stressor based on one's spiritual beliefs. An individual can make meaning out of the situation through causal attributions to God's will or God's plan. When an individual attempts to find meaning in a situation, distress may be reduced (Siegel \& Schrimshaw, 2002) enough to facilitate engagement in coping behavior. Spiritual coping encompasses particular behaviors that an individual uses to respond to the stressor (problem-focused) or related emotional reactions (emotion-focused).

In conclusion, spirituality and religion can provide meaning in circumstances involving a specific person-environment transaction (Park \& Folkman, 1997). In a transactional model of coping, meaning making is a cognitive reassessment that is essential to successful adaptation in circumstances that are chronic or not easily improved by coping efforts (Park \& Folkman, 1997). Not being able to find meaning is associated with doubt, uncertainty, and psychological distress, which inhibit effective coping behaviors (Emmons, 1999).

Spirituality is both distinct and personal and universal to all human beings, regardless of age or stage of development (Kenny, 1999). A child can have an advanced awareness of spiritual concepts without ever having been part of a religious group (Heilferty, 2004). Spirituality is important in helping children create values and beliefs that influence their behaviors (Burg, Mayers, \& Miller, 2011). Spirituality may play an 
important role in meaning-making with regards to attitudes and beliefs about the world, self, and others (Park \& Folkman, 1997). A spiritual process of meaning-making can relate to all aspects of life such as interpersonal relationships, work, general philosophy of living, attitudes, and/or the individual's concept of God or higher power (Pargament, 1997).

Research has shown an association between spirituality and positive coping with cancer and its treatment (Purow, Alisanski, Putnam, \& Ruderman, 2011). Children with cancer try to make sense of their illness, suffering, and impending death by searching for answers beyond this material world. To decrease their distress, they may express their spirituality through music, art therapies, therapeutic play, and stories related to illness and dying. Children express their spirituality when they pray, sing songs, or hold hands for blessings (Yust, 2003).

In their study of the spiritual well-being (existential and religious well-being) of 67 adolescents (ages 11 to 19 years) with inflammatory bowel disease (IBD) versus 88 healthy peers, Cotton et al. (2009) examined associations of spiritual well-being with mental health outcomes (emotional functioning and depressive symptoms) and the impact of existential versus religious well-being on mental health. The mean age of the participants was $15.1(\mathrm{SD}=2.0) ; 80(52 \%)$ were male, and $121(78 \%)$ were White (Cotton et al., 2009). Demographic characteristics were similar between the adolescents with IBD and the healthy peers. There was a fairly even distribution of severity of IBD illness: 20 (30\%) with moderate severity, $26(39 \%)$ with mild severity and $20(30 \%)$ with inactive disease. The mean time since diagnosis was 3.1 years. Adolescents with IBD had greater depressive symptoms than their healthy peers but similar levels of emotional functioning. 
Levels of depressive symptoms and emotional functioning were similar among adolescents with mild, moderate, and inactive IBD symptom severity. Of the 154 adolescents, 91 (59\%) reported that their relationship with a Higher Power/God contributes to their overall well-being. The majority of the adolescents felt optimistic about their future (82\%) and said that a Higher Power loved them $(81 \%)$.

Similar to other studies of spiritual well-being (Wong, Rew, \& Slaikeu, 2006; Cotton, Larkin, \& Hoopes, 2005), existential well-being (meaning and purpose in life) was more strongly related to decreased depressive symptoms and increased social functioning than religious well-being (connectedness to the sacred) was (Cotton et al., 2009). Disease severity moderated the relationship between spiritual well-being and mental health outcomes. There was a stronger positive relationship between existential well-being and emotional functioning for adolescents with IBD than for their healthy peers. Greater religious well-being was related to decreased depressive symptoms for adolescents with IBD, but not those without the condition. Longitudinal studies with adolescents with IBD and other conditions such as sickle cell or cystic fibrosis are needed to determine whether relationships between the variables change over time.

Spiritual and religious coping may provide children and adolescents with a way to make sense of a traumatic experience (Bryant-Davis et al., 2012). In a study by BryantDavis et al. (2005) of 70 African American men and women who survived childhood violence, $55 \%$ of the participants used spirituality to cope with the traumatic event, and $52 \%$ used community support. Both spirituality and community support included religious organizations which may have affected the results. Attending church was categorized as a community resource not as the use of spirituality. 
Barnes et al. (2000) found that a child's sense of spirituality may provide a basis for positive coping. In a study by Bernstein, D’Angelo, and Lyon (2012), adolescents with HIV used religion/spirituality as a coping mechanism which generated hope and comfort. A study by Lyon, Garvie, He, Malow, McCarter, and D’Angelo (2012) found that spirituality in African-American HIV positive adolescents was associated with fewer symptoms of depression and anxiety and increased quality of life. Spirituality did not vary as a function of age, gender, or stage of illness.

This study focused on the spiritual coping strategies used by children after the death of a parent, grandparent, or sibling and the relationship between children's spiritual coping strategies and grief, personal growth, anxiety, and depression (Figure 1). The death of a parent, grandparent, or sibling was the stressor. The person factors consisted of the bereaved children's characteristics which were hypothesized to have a direct effect on their spiritual coping strategies and level of grief. Spiritual coping strategies may aid children in their interpretation, comprehension, and reaction (Bryant-Davis et al., 2012) to the death and have a direct effect on their grief, personal growth, anxiety, and depression. Children's grief was hypothesized to have a direct effect on their levels of personal growth, anxiety, and depression. 
Figure 1. The Spiritual Framework of Coping after a child is bereaved of a parent, grandparent, or sibling.



\section{Research Questions}

This study addressed the following research questions:

1. Are there differences in the spiritual coping strategies used by children across age groups, gender, race/ethnicity, participation in religious rituals and practices, and the relationship of the deceased to the child (parent, grandparent or sibling)?

2. Are children's spiritual coping strategies related to their grief, personal growth, anxiety and depression after the death of a parent, grandparent, or sibling? Is 
children's grief related to their personal growth, anxiety, and depression after the death of a parent, grandparent or sibling?

3. Do these relationships hold when child's age, gender, race/ethnicity, participation in religious rituals and practices, and relationship of the deceased are controlled? 


\section{CHAPTER II}

\section{REVIEW OF THE LITERATURE}

In 2016, the Centers for Disease Control and Prevention determined that in 2014 the United States had a total of 23,215 deaths that occurred in infants under age one year. The annual death rate among children was 3,830 per 100,000 (for children 1 to 4 years); 5,250 per 100,000 (for children 5 to 14 years); and 9,586 per 100,000 (for adolescents 15 to 19 years) (Centers for Disease Control and Prevention, 2016). In 2014 there were a total of 2,626,418 adult deaths reported in the United States (Centers for Disease Control, 2016). The high adult and child mortality rates indicate that many children are bereft of a parent, grandparent or sibling and ultimately have to cope with such a loss at a time where they are most vulnerable due to the developmental, psychological, and cognitive changes they are experiencing.

Research has focused on how youths use religious coping when dealing with stressors such as serious physical illness like asthma (Benore, Pargament, \& Pendleton, 2008), sexual assault (Frazier, Tashiro, \& Berman, 2004), and depression (Carleton, Esparza, Thaxter, \& Grant, 2008). Religion is often used to explain events, such as death, which are beyond human understanding (Ungureanu \& Sandberg, 2010). Religion gives meaning to situations and life events that humans fear and don't comprehend. It serves as a path for human behavior and thinking, making it possible to find meaning in situations that are difficult to understand (Bryant-Davis et al., 2012).

Spirituality and relationship to a higher power have also been found to be the most robust protective factors against health damaging behaviors in adolescents (Burg et al., 2011). For children who are old enough to understand that they are dying, belief in 
God and religious teachings may help them make sense of and cope with their situation (Purow et al., 2011). This chapter reviews the literature on children's understanding of religion, religiosity, and spirituality from a developmental perspective and factors related to development of religion, religiosity, and spirituality, children's responses to parent, grandparent, and sibling death (grief and bereavement), and cultural differences in death rituals for deceased children and adults.

\section{Religion and Spirituality}

According to Good et al. (2011), religion is a multidimensional construct that pertains to institutional religious expressions such as dogma and ritual, as well as personal religious expressions such as spirituality, beliefs, and religious practices. Spirituality is different from religion because it is not based on a discrete value system (Farrell et al., 2008). In contrast, others argue that even though spirituality and religiosity have distinct characteristics, they are highly intertwined (Good et al., 2011; Walker, Reid, O'Neill, \& Brown, 2009). Atheists and agnostics may categorize themselves as spiritual despite not participating in any organized religion (Cotton et al., 2006).

\section{Children's Development of Understanding about Religion and Religiosity}

Fowler (1981) suggests that the development of children's faith occurs in stages consistent with Erikson's theory of psychosocial development, Piaget's theory of cognitive development and Kohlberg's theory of moral development. These theories provide a scaffold that portrays how children's thinking becomes increasingly abstract which enables comprehension of religious concepts (Barnes et al., 2000). The advancement of children's spirituality is parallel with cognitive development and is developmentally sequenced. 
Fowler's faith development theory. Fowler's faith development theory is unique because his stages have very few boundaries (Neuman, 2011). There are minimums before which certain stages cannot occur, but there are no maximums. Only the first three of the six stages are relevant to children and adolescents.

Fowler states that faith development begins when there is a "convergence of thought and language" (Fowler, 1981, p. 121) and when speech is more developed. Children in stage 1, Intuitive-Projective Faith (Neuman, 2011), are typically 3 to 7 years old. During this stage children do not fully understand cause and effect and reversibility. Children in this stage integrate stories and images about the divine from their parents and culture into beliefs about God.

Stage 2, Mythic-Literal Faith, begins with an increase in concrete operational thinking common in elementary school children from 7 to 12 years of age. Children can now understand cause and effect relationships, take the perspective of others, and narrate stories. Individuals outgrow stage 2 when they begin to realize that bad things happen to good people (Fowler \& Dell, 2004).

Stage 3, Synthetic-Conventional Faith (12 to 21 years), begins with the transition to formal operational thought which makes it possible to reflect on one's own thinking (Neuman, 2011). Individuals in this stage can process hypothetical situations, work with symbols, and be idealistic. They can step outside the box and see new connections. Fowler's model is more spiral than linear, with some qualities overlapping during transitions through stages and some backsliding during crises of faith (Fowler, 1981).

Kohlberg's theory of moral development. Inspired by Piaget, Kohlberg (1981) concluded that moral reasoning develops in three predictable levels: pre-conventional, 
conventional, and post-conventional (Vasta, Miller, \& Ellis, 2004). An important feature of this model is how the social and moral components in each stage interact.

The social perspective component focuses on the point of view from which the moral decision is made and the moral content is strongly influenced by the child's experiences with moral situations. The individual moves from one stage to the next in order, without skipping any of them. Kohlberg' assumes that development is a result of the improvement of cognitive skills and recurrent encounters with moral issues (Vasta et al., 2004).

\section{Children's Religious Beliefs and Practices}

Children's families provide the religious context of their lives (Neuman, 2011). Braswell, Rosengren, and Berenbaum (2012) investigated the relationship between parents' and their children's beliefs in science, religion, and the paranormal. The sample consisted of 509 parents of children between the ages of 3 and 7 years. The majority of the parents were White $(66.7 \%)$ with some (64.9\%) higher education. As expected, the parent's own beliefs are associated with the beliefs they report cultivating in their children. However, the researchers did not directly measure children's beliefs, relying solely on parent reports of their child's current beliefs. Children's reports of their beliefs would provide a clearer understanding of the association between their beliefs and their parents' beliefs.

Schottenbauer, Spernak, and Hellstrom (2007) examined the influence of family religiosity and religious behaviors on multiple areas of children's wellbeing (general health, social skills, academic achievement, and behavior problems) and the relative contributions of religious and non-religious variables to parenting style. This study 
analyzed data from the National Head Start-Public School Early Childhood Transition Demonstration Study (NTDS) (Ramey et al., 2000); a multisite, randomized trial of Head Start services (developmentally appropriate curricula, nutrition, health, parent involvement, and social services) from kindergarten through third grade.

In third grade children, Schottenbauer et al. (2007) found that family attendance at religious/spiritual programs was significantly correlated with improved child vocabulary, reading, math, social skills, physical health, and mental health problems. However, parental trust in a higher power predicted more negative child behaviors, such as more externalizing behaviors and hyperactivity.

A qualitative study by Takritti, Barrett, and Buchanan-Barrow (2006) of 58 Arab Muslim, Asian Muslim, Christian, and Hindu children between 5 and 11 years of age living in North London investigated children's understanding and the importance of religion. Results demonstrate that religion is highly salient and that children's religious identity is subject to a complex pattern of influences which cannot be exclusively explained by age or cognitive differences. This study should be replicated in adolescents to determine if results remain consistent in older children.

Zehnder, Vollrath, and Landolt (2006) examined the effects of various coping strategies on children's port-traumatic stress symptoms and behavioral problems one month after their accidental injury or diagnosis of a chronic disease and again 1 year later. The sample consisted of 161 German-speaking pediatric patients, 6 to 15 years of age, in Switzerland. Children who used religious coping reported decreased symptoms of post-traumatic stress disorder 12 months after an accidental injury or the diagnosis of a chronic disease. The researchers conclude that praying to God increases hope and 
decreases feelings of loneliness and that religious coping may be associated with family functioning which may mediate psychosocial adjustment. Differences between the accidental injury group and the chronic/long-term illness group were not reported.

A retrospective study by Gunnestad and Thwala (2011) focused on the relationship between religion and resilience in children and youth in difficult situations. Data were collected from students in Zambia who wrote about a difficult time in their childhood and how they were able to overcome it. Orphans in schools in Swaziland also were interviewed about their situations and needs. The investigators found that a number of elements in religion such as prayer and intercession, fellowship, faith and hope, values and good models, in addition to counseling were associated with resilience in children and youth. Spirituality or religious practices may act as protective factors for children experiencing emotional assaults of racism, abuse, destruction of one's culture, or living as a refugee (Gunnestad \& Thwala, 2011). In some cases, situations with a negative religious interpretation could be turned into deeper and positive religious coping if the child received assistance to understand and positively redefine the situation.

\section{Age and Gender}

Children approach religion in a practical manner by creating their own tradition, often based on the parents' religion, which assists them with their own needs (Ridgely, 2012). Children develop hybrid traditions from their inherited faith and the practices and images they learn from friends and the media (Pike, 2009). A study by Schwartz (2006) explored the distinctive and complementary roles of parents and friends in predicting adolescent religious faith. The sample consisted of more than 4,000 Christian adolescents aged 14 to 18 attending an international denominational Christian youth conference in 
Toronto. Schwartz (2006) found that perceived support of faith by friends mediated the influence of similar parental support on adolescent's religious beliefs. Adolescents who see in their parents consistent and powerful examples of Christian lifestyle are more likely to express a stronger integration of faith in life, belief in God, and participation in spiritual acts (Flor \& Knapp, 2001; King, Furrow, \& Roth, 2002).

In their study of children's early knowledge about connections between prayer and emotion, Bamford and Lagattuta (2010) compared thought processes of adults to those of young children with a sample of: 20 four-year-olds, 20 six-year-olds, 20 eightyear-olds, and 40 adults (18 to 41 years). The adult sample was 50\% Asian, 25\% Hispanic, $25 \%$ Caucasian, and $25 \%$ other, but $85 \%$ of the children were Caucasian. Results of the study (Bamford \& Lagattuta, 2010) reveal that by age 8, children associate praying with negative emotions and feeling better after praying. By age 6 , they viewed prayer as a mental activity to communicate with God. Level of religious activity was not related to the concept of prayer held by 6-year-olds, 8-year-olds, or adults, but it did predict 4-year-olds' knowledge that negative emotions motivate prayer and that individuals pray to give thanks after positive events. Level of religious training also predicted 4- and 6-year-olds' understanding of the concepts of God and prayer. Parents report instilling their own beliefs in their children (Braswell et al., 2012).

Positive emotions were the primary motivators of prayer for younger children (ages 4 and 6), and negative emotions were the primary motivators for older children (age 8) and adults (Bamford \& Lagattuta, 2010). When taking into account religiousness and spirituality in the children's backgrounds, younger children with high exposure to religious training had more sophisticated prayer and God concepts, suggesting that 
religious and spiritual experiences may enhance the development of children's concepts of God and prayer.

Limitations in this study (Bamford \& Lagattuta, 2010) are that the majority of children were Caucasian, but the majority of adults were Asian. This difference in the race/ethnicity, and probably religion, could have affected the results. Longitudinal research with more advanced prayer concepts (the specific mechanisms by which humans communicate with God and the specific processes by which prayer can regulate emotions) is needed to increase our knowledge of the relationships among religious training, knowledge about prayer, and children's development of knowledge about the mind and emotion.

Dew et al. (2010) investigated whether religion/spirituality decreased depression and substance abuse and enhanced social support with a sample of 145 adolescents, 12 to 18 years of age, from two psychiatric outpatient clinics. Religious affiliations of the adolescents were Conservative Protestant (71\%), Liberal Protestant (15\%), Roman Catholic (7\%), other (5\%), or none (5\%). Ethnic composition of the sample was as follows: Caucasian (56\%), African American (35\%), and other (9\%). Adolescents completed the Beck Depression Inventory-II (BDI-II), the Fetzer multidimensional survey of religion/spirituality, and inventories of social abuse and social support. These measures were repeated 6 months later. Adolescent psychiatric patients with more depressive symptoms and a poorer prognosis reported a loss of faith. Since the researchers did not include measurements of the adolescents' faith before they were diagnosed with depression a sample of normal controls should have been included. 
In another study, Cotton et al. (2005) examined the effects of spirituality and religion on depressive symptoms and health risk behaviors with 134 primarily White adolescents from a suburban high school. Most adolescents (89\%) reported a belief in a Higher Power/God, and 77\% stated that religion was important in their lives. Higher spiritual well-being was related to fewer depressive symptoms and risk-taking behaviors. However, adolescents who felt that religion was important to them had more depressive symptoms. Psychosocial variables (family and peers) known to be related to depressive symptoms and high risk behaviors were not measured in this study. Longitudinal studies are needed to determine the direction of effects between religion and depression. Perhaps, adolescents stressed by their religion became depressed or they blame God for their depression. It may also be that depressed adolescents turned to religion for support. The religious affiliations of the participants were not specified.

Bosacki et al. (2010), with data from a larger study of 535 Canadian children (281 girls, 254 boys; 5-13 years of age) on sense of self and media habits (Elliot, Bosacki, Woloshyn, \& Richards, 2002), explored the role of religiosity and/or spirituality in children's self-reported media preferences and habits over a three-year period. The children were from 14 culturally diverse schools in Ontario. English was the children's main language.

In the study (Bosacki et al., 2010), children completed a 22 page self-report questionnaire about aspects of their daily lives ("What activities are done for fun?") and their use of popular media ("What is your favorite TV show?; "What do you do on your computer?’). Grade 1 children were assisted by grade 7 reading buddies who read the 
questionnaires to the younger children. Children's responses were then analyzed for themes that referred to or included issues of spirituality and religiosity.

Across all ages (excluding 6-year-olds) and modes of media (excluding videos and magazines), girls were more likely than boys to discuss issues of religiosity and spirituality (Bosacki et al., 2010). The only grade where boys provided more than half of the spirituality codes (56\%) was Grade 1 (6-year-olds).

Issues of spirituality and religiosity were most likely to surface during weekend and afterschool activities (church and Sunday school attendance), parents' reading habits (especially mothers who read the Bible), and choice of films and television programs related to God and religiosity (Bosacki et al., 2010). This suggests that families play an important role in children's understanding of spiritual and religious experiences. Children's beliefs and practices are shaped and transformed in their interactions with parents, teachers, or friends, and through books, television, and the internet (Ridgely, 2012).

Methodological limitations include lack of explicit questions involving religiosity/spirituality may have influenced their responses. Future research should explore the connection between language ability and religious/spiritual experiences as well as the role of gender in religious and spiritual development.

The study by Bosacki et al. (2010) found a connection between religiosity/spirituality and engagement in both social (going to church with the family and religious material read to the children by their mothers), and individual activities such as listening to music and reading books, which suggests that children's perceptions and experiences of religiosity and spirituality are both social and solitary. 


\section{Race/ethnicity}

Through semi-structured interviews and drawings, Cotton, Grossoehme, and McGrady (2012) studied the types of religious coping used by 20 young AfricanAmerican children (ages 5 - 10 years) with sickle cell disease, the type and frequency of prayer, and their view of God/Higher Power. The children used their religious beliefs to have a sense of control when they were sick or hospitalized. These children found comfort and closeness to the divine in times of crisis through their religious beliefs.

More than half of the children reported using prayer or religion to help them cope with the diagnosis of sickle cell disease and its physical effects. The majority of the children saw God as a functional God that helped them heal. They also saw God as having a supportive role because He was there for them when they needed Him. Research suggests that religious and spiritual beliefs help an individual cope with and recover from illness and also prevent mental and physical illness (Josephson \& Dell, 2004; Koenig, 2003; Mueller, Plevak, \& Rummans, 2001). This study did not address the pattern of religious coping in the parents of these children which is an important element to consider in children with a chronic illness. Perhaps children with a chronic illness use religion as a coping mechanism since they see their parents coping with the disease in the same manner.

Benore et al. (2008) studied the relationship between positive religious coping strategies and adjustment in 87 primarily African American and Baptist children and adolescents (ages 8-17) hospitalized for asthma. Children newly diagnosed with asthma were excluded. Contrary to most studies, positive religious coping was related to poorer adjustment. Perhaps this means that positive outcomes of coping occur over time. When 
these variables are examined early in the coping process there will be a negative association between positive religious coping and poorer adjustment (Benore et al., 2008). The positive outcomes of religious coping may not be evident until a later time. Adjustment prior to the hospitalization for asthma was not measured.

Peer relationships become increasingly important in adolescence (Geckova, Pudelsky, Tuinstra, 2000). Adolescents who frequently attend religious services may develop a social network of similar adolescents safeguarding against depression (Pearce et al., 2003), teen pregnancy, and sexual permissiveness (Corwyn \& Benda, 2000; McCree, Wingwood, DiClemente, Davies, \& Harrington, 2003).

Pearce et al. (2003) examined the relationship of depressive symptoms to indicators of religiosity (attendance, private religious practices, and self-ranked religiousness) and interpersonal religious experiences (positive support from one's congregation and frequency of negative interactions with one's congregation) in 744 adolescents (50\% female and 82\% White) in Grades 7 to 9 (Mean age: 13 years, SD= 0.45). The researchers also examined the moderating influence of sex and ethnicity on relationships between religiousness and depressive symptoms. Adolescents' reported religious affiliations were $61 \%$ Catholic, $13 \%$ Protestant, $6 \%$ Jewish, $10 \%$ other, and $10 \%$ no religious affiliation.

The three dimensions of religiousness accounted for a small yet significant amount of variance in depressive symptoms controlling for demographic variables (Pearce et al., 2003). More frequent attendance and higher self-ranked religiousness were correlated with fewer depressive symptoms. Positive interpersonal religious experience was associated with lower depressive symptoms, and negative interpersonal religious 
experience was associated with greater depressive symptoms. Adolescents' psychological wellbeing was more strongly associated with their social experience in religious environments than with their attendance at religious services. In this sample, sex and ethnicity did not moderate the relations between the dimensions of religiousness and depressive symptoms.

This study (Pearce et al., 2003) consisted of relatively non-depressed adolescents of only two ethnicities, European American (82\%) and African American (18\%). Studies of adolescents with higher risk for clinical depression and greater ethnic diversity are needed. Also, social support in general was not examined, only religious support. Prior studies have found a significant relationship between social support and religious activity (Pearce et al., 2003).

\section{Religion}

Judaism. An article by Wahlhaus (2005) recognizes that there is a difference between Jewish law and custom is often unclear, specifically in relation to death. Walhaus (2005) acknowledges that in the frenzied aftermath of bereavement, support and refuge are provided to the mourner through the framework of ritual and the importance on the ongoing relationship that is part of the Jewish burial and mourning process and the corresponding community involvement. It is important to understand that other movements related to Judaism such as Conservative and Reform may share similar views regarding death rituals yet they can have different daily practices (Boylan, 2005).

If a baby who has not lived for thirty days is not seen as a fully viable human being in the Jewish religion, it is not an obligation to perform the full traditional mourning rituals (Sparshott, 2004). If the baby is stillborn or dies before 30 days, the 
parents can choose to have the rituals and traditions surrounding the death performed. After the person dies the Rabbi of the person or the family, or both is contacted and the Chevra Kaddisha, the Burial Society is informed. The Chevra Kaddisha is in charge of the ritual preparation of the body for burial (Loike, et al., 2010). The body is handled with enormous respect and care. After death the windows are opened (to ease the departure of the soul), eyes are closed, jaw is bound, and limbs are straightened. The body is placed on the floor (close to the earth which is its origin), feet pointed towards the door (to allow the soul to depart), covered with a sheet (for respect, a candle is placed close to the head (to illuminate the journey of the soul).

According to Jewish tradition, death is seen as a threshold between this world and the world to come. The Torah (Jewish bible) explains that people are made in the image of God and their souls return to God when they die. Wahlhaus (2005) feels that rituals that "liberate the soul of the dead body" (for example, the deceased's feet are pointed towards the door) are symbolic of this belief and positively assist the mourner psychologically. These rituals serve as a symbolic expression of an internal expectation that the soul lives on in another dimension. Containers in the home filled with water are emptied (this is done since water symbolizes life and pouring it out indicates the end of life and announces a death in the home).

When the body is prepared for the funeral the body is washed according to Jewish ritual (Taharah), clothed in simple white linen shrouds (Tachrichim), and buried in a simple wooden coffin since everyone is seen equally in the eyes of God. Jewish law mandates that until the body is buried it is not left alone and it is guarded by a watcher (kevod hamet, honoring the dead) (Prosser, Korman, \& Feinstein, 2012). The preparation 
of the body and its guarding is the responsibility of the Chevra Kaddisha members. There are family members that want to watch over the body as a sign of respect (Sable, 2009). These last respects are fundamental to the mourners because they recognize both their emotional relationship prior to their loved one's death and their active involvement in the wellbeing of the deceased.

Funerals are conducted in a simple fashion. Traditional Jews anticipate the coming of a messiah during which time the dead will be resurrected, suffering on earth will be justified, and peace and harmony will triumph among the righteous. For Orthodox Jews it is critical for the deceased to be buried in the ground in anticipation of resurrection. Due to the latter cremation is not acceptable (Dimond, 2004). In the Jewish religion, the body should be protected from desecration.

After the individual dies the burial is done as soon as possible within twenty-four hours. Before the funeral the Keriah or rending is done (Wahlhaus, 2005). This is a ritual that comprises of tearing off a piece of clothing worn by the mourner (blouse, shirt), on the left above the heart if the deceased is a parent, on the right for other relatives. This is the first symbolic representation of grief by the mourner of the wound that death leaves behind, which can never be repaired. Wahlhaus (2005) proclaims that this ritual's psychological benefit is in the therapeutic expression and perceived visual image of the wound which prevents damage to oneself.

At the moment of keriah, (or when the individual dies), the blessing "Blessed art Thou, O Lord our God, King of the Universe, the true judge (dayan ha'emet)" is performed (Wahlhaus, 2005). This prayer serves as a reminder to the mourner how miniscule they are in the larger scheme of things. This prayer reminds the mourner that 
death is a happening that is beyond their control and it affirms trust in God that he will judge the deceased accordingly. This prayer also brings comfort to the mourner because even though the relationship with the deceased is no longer present the relationship with God is ongoing.

The Jewish burial is simple and noble. It entails the eulogy and the Kaddish, the traditional Aramaic prayer for the dead. The eulogy is done by the officiating Rabbi or by members of the family. The Orthodox custom only allows males to deliver the eulogy at the funeral. The eulogy cannot be done during certain dates such as Rosh Chodesh, during the first seven days of Sivan, on the days before Rosh Hashanah and Yon Kippur, on Chanukah, and on Purim (Wahlhaus, 2005). The eulogy celebrates the life of the deceased. Mourners are allowed to cry and express their emotions and grief.

The mourner's Kaddish is the traditional prayer for the dead and it is a declaration of faith in God. This prayer is said at the graveside or once the attendants have returned to the chapel. This prayer is traditionally said by the male members of the family only in the company of a quorum of ten men (a minyan), which are representative of the community. Wahlhaus (2005) states that psychologically, after the prayer is recited, the bereaved feels that they have contributed to the liberation of the soul of the deceased.

In the Jewish tradition, the mourners and attendants at the funeral fill the grave with earth. Each individual, if they wish, shovels three spadesful of earth in the grave (males only in the Orthodox practice). This ritual symbolizes closure. The principal mourners then walk through two aisles created by the attendants, a corridor of consolation created by the community. Psychologically, this is a metaphor for a walk back into life; the corridor represents the birth canal. Attendants wash their hands to 
represent the removal of the impurity of the death before entering the chapel once more to conclude the service. It is tradition for mourners to not dry their hands in order not to wipe away the memory of the deceased. Even though washing hands represents a purity ritual, washing the hands represents a psychological preparation for change, symbolizing a new beginning. Living without the deceased is a new beginning.

After the funeral, the mourner begins a period called shiv'ah which lasts for seven days (Wolowelsky, 1996). The mourner does not go to work for seven days and they sit on low stools, humbled and destitute by the bereavement. They perform basic daily hygiene without lavishness, and men abstain from shaving. They wear the garment which was previously torn, they do not purchase or wear new clothes, and they do not cut their hair. Also, they do not watch television or listen to music, and they abstain from having sex. A memorial candle is lit throughout the seven days of shiv'ah, and mirrors are covered and turned to face the wall. Wahlhaus (2005) describes that this is a time of reflection for the mourner to think about his identity, rather than to face a reflection of their bereavement and seclusion.

During shiv'ah the visitors take care of the mourners, provide them with food and necessities and tend to their wishes. The mourners do not greet the visitors; instead, they wait for the mourner to start a conversation. The community takes care of the mourner. The mourners do not observe shiv'ah on Shabbat or holy days. Having relatives and friends around during shiv'ah assist with the outward expression of grief and can help with illogical or distorted feelings of guilt.

The shiv'ah extends into a phase called the shloshim, which is a period of thirty days after the funeral. Community support decreases during this time; mourners wear 
fresh clothes, uncover the mirrors and go back to work. The anniversary of the death is taken into account on Yahrzeit each year, depending on the corresponding date on the Hebrew calendar. A memorial candle is lit for 24 hours on Yarhzeit each year.

The mourner believes that this light is to show the departed soul that they are still remembered. After this, the Kaddish is recited in the synagogue on each anniversary of the death of a parent. Most of the people visit the grave of the deceased on Yahrzeit and they place a small stone on the grave as a representation of their visit. Yizkor memorial prayers for the dead is said four times a year, on Yom Kippur, Shemini Atzeret, the last day of Pesach and the second day of Shavout (Whalhaus, 2005). Participating in Yahrzeit and reciting Yizkor are the only two ongoing rituals of the bereavement.

Islam. A study by Suhail and Jamil (2011) explored the bereavement process and continuing bond in Pakistani Muslims focusing on how these processes are influenced by culture and religion. Ten participants ( 7 men and 3 women) were interviewed and their interviews were analyzed. They ranged in age from 18 to 65 and they had experienced the loss of a significant relative (not a child) within the past 14 months to 5 years. Results indicated that that culture and religion had a strong influence on the expression and intensity of grief. The participants maintained strong bonds with the deceased by participating in religious rituals, such as praying, reciting holy verses, visiting graves, and arranging communal gatherings. Such bonds are seen as adaptive by researchers (Epstein, Kalus, \& Berger, 2006).

Islam is a monotheistic religion as is Christianity and Judaism. It is important to differentiate Islam and Muslims. Islam refers to the religious principles that are followed 
(derived from the Quran and tradition) and the Muslims are not a community that can be depicted as one, hence, they cannot be easily defined in this context (Venhorst, 2012).

There are no priests or clergy in Islam. An Imam, an educated member of the community, often is in charge of prayer sessions in the community (Ross, 2001). Muslims do not view death as a final. Hedayat (2006) states that "death in Islam is an active process, a transition for the soul from the material world to a spiritual world of purgatory" (p. 1285). They believe in an afterlife from the time that the individual dies until the time they are resurrected. The dead wait for the Day of Judgment in their graves.

When the person is almost dying he or she is positioned supine, facing Mecca (east) (Dimond, 2004). The room is kept fragrant with the use of perfumes. Anyone who is menstruating or not clean needs to leave the room. Passages of the Quran, Muslim scriptures, are read by the dying individual or a close family member. The Shahadah, the principal ideology of Islam, is recited in the ear of the dying, "There is no God but Allah and Muhammad is his prophet" (Kramer, 1988). This is the most important statement of Muslim faith. This is the first words heard by a Muslim newborn and the last words heard upon their death as the family turns the face of the dying towards Mecca (Kassis, 1997).

In medieval Muslim societies a special type of literature was created for consolation from the death of an infant or child (Hedayat, 2006). This literature included three elements which are the virtue of patience (Muslims are encouraged to bear problems with fortitude, intercession (a child or infant who dies may intercede on their parent's behalf so they could gain entrance into heaven), and substitution (the death of a child may be so another child can be given to the parents which will be more religious). In Muslim tradition, a baby who dies at or just birth must be given a name (Sparshott, 
2004). After death of an infant or child the following rituals should be performed within 24 hours after death: washing, anointing, shrouding, praying, and burying the corpse (Hedayat, 2006). The mother cannot perform the ritual washing in the immediate postnatal period due to her lochial loss since this ritual is considered an act of worship (Arshad, Horsfall, \& Yasin, 2004).

Families prepare the deceased body for burial immediately after the individual dies (Abu-Rabia \& Khalil, 2012). In the Islamic tradition the dead are buried on the same day of their death or on the following day if their death occurs late. The deceased's eyes and mouth are closed, the feet are tied, and the body is covered with a sheet.

Close family members of the deceased that are of the same gender perform ritual washing of the body with water and perfumed leaves (Venhorst, 2012). The body is washed beginning with the right side, which is part of Islamic tradition. The first body parts that are washed are the hands, arms, mouth, nostrils, and feet. During this ritual, the body is never fully uncovered. After the body is washed it is wrapped in one or two cotton shrouds and placed in a simple wooden coffin to be taken to the burial site. Embalming is prohibited in Islamic tradition.

Islamic family members are present when their loved one is dying and at the time of death to mourn and to prepare the body for burial. Mourning is permissible in the Muslim culture but wailing and other such acts are not accepted and this is why women in the Muslim culture are not permitted to follow a funeral procession to prevent such an open display of emotion (Abu-Rabia \& Khalil, 2012).

The Quran may not be read near the corpse but people may offer their personal prayers. These prayers have to be done while standing and tradition also mandates for 
participants to remain standing during a funeral procession until the body is buried. The Quran does not mention funeral practices. Burial practices have been instituted by religious jurisprudence and are considered religious rituals not social reunions.

It is seen as an honor to carry the coffin in the cemetery. During the funeral coffin bearers repeat the phrase "God is great, God is merciful" (Kramer, 1988). A close relative of the deceased presides at the funeral, standing by the head of the deceased if male or by the trunk if female. The funeral attendants recite specific prayers and statements.

After the ritual prayers, the body is removed from the coffin and buried into a 6foot deep grave which can be a hole or trench facing Mecca. Before the body is lowered into the grave, mourners may whisper instructions for the "trial of the grave" in which the two angels visit to question the dead about his or her life. The shroud is removed, the face is turned towards Mecca and shadahah is whispered once more into the ear of the deceased. Earth is placed loosely over the body and each participant places three handfuls of dirt on the grave. The dirt is placed loosely over the cadaver so that the deceased can sit up when the angels come to question him or her.

Hinduism. Hinduism is a distinct religion because its roots do not arise from a single scripture, founder, or sacred place; instead, Hinduism encompasses a set of philosophies cultures, and way of life (Clements et al., 2003). The only uniformity is found in the approach to death. Hindu's believe in the laws of Karma and they feel that each birth is associated with actions taken in previous births (Lobar et al., 2006). Hindus' partake in a cycle of birth and death in order to transcend through the accumulation of good Karmas (actions), with the end result being the liberation of the soul. The end result is that they attain enlightenment (Nazarko, 2006). 
Hindus believe that the soul enters the body of the baby when the woman is seven months pregnant. If the woman miscarries before this time there are no mourning requirements (Sparshott, 2004). Mourning for a dead child is only done for 10 days because after this the soul re-enters the life cycle.

When a Hindu dies their bodies are bathed, massaged in oils, and dressed in new clothes. The body needs to be cremated before the next sunrise. The cremation facilitates the soul's transition to the other world. Rituals are performed for 10 days after the death which is a time were intimate prayer and meditation is instituted to ease the transition of the departed soul to the next world. The death rites have to be meticulously performed if not the deceased will remain a bodiless ghost and will not be incorporated as an ancestor (Boer \& Zock, 2004). The Hindu's believe that the soul is watching over them during the 10 days and on the 11th day it releases its attachment to the former life. The family starts to reunite with friends and community members only on the $12^{\text {th }}$ day to honor and keep their loved one's memory alive.

Buddhism. Buddhism has had an important influence on Chinese culture for thousands of years (Hsu, O'Connor, \& Lee, 2009). In the Asian culture, when an infant or child dies they are profoundly mourned (Lobar et al., 2006). The family members dress in white clothing or wear white head bands.

After a person dies, the body remains in the bedroom or house chapel until its disposition during the final ritual. The body is covered except for the top of the head. Family members and friends set up a small shrine consisting of pictures of Buddhas or empowering deities. The body is positioned on its right side. Incense is burned as an offering and it is also used to mask the odor of body decomposition. Offerings are made 
outside the house or death chamber to persuade the deceased to leave his or her body. The body is washed by monks or family members in preparation for the burial and it is handled in a respectful way (Dimond, 2004). Cremation is the common method for disposing of the body. Ritual disposition of the body is done from day 4 to 10 .

Funeral rites take 49 days to complete and this period marks a transition of consciousness between death of the body and rebirth into another body. Sometime during the 21 days the lama performs the transference of consciousness ritual. Barley and butter, used as offerings, are placed on the corpse's head and the lama guides the deceased on how to break attachment to the body since Buddhists believe that the consciousness of the dead person is seven times clearer than the consciousness of a living person (Goss \& Klass, 1997). Instructions are read to the dying person and later to the corpse by a lama (spiritual teacher) to help guide it through the transitional state between life forms (Goss \& Klass, 1997). The objective is to prepare the consciousness of the dying person for the opportunity of enlightenment that is available in the future.

In Buddhism, the state of mind of the person at the moment of death will affect the process of rebirth. In the Buddhist religion, chanting the teachings of the Buddha will have a calming effect on the mind of a dying individual (Goss \& Klass, 1997). The above rituals also help prepare the dying person to transition from death to rebirth. The person has better chances of a good rebirth if the state of the mind is positive.

Christianity. Christians believe that there is life after death. There are many denominations but, for the most part, the majority of Christians are either Roman Catholic or Protestant. Religion and spirituality are of upmost importance for Latinos 
who practice Catholicism which is the dominant religion for this group (Clements et al., 2003).

In the Catholic religion humanity is born sinful and this is why some Christians believe that the Sacrament of Baptism is the beginning of an individual's relationship with God. The Sacrament removes the individual's state of original sin. If a newborn dies before being baptized anyone can perform a baptism as long as they themselves are a believer (Sparshott, 2004). On the contrary, Leever, Deegan-Krause, Richard, Kenneth, Do, and Peggy (2004) report that baptizing of deceased infants is inconsistent with Catholic doctrine and the National Association of Catholic Chaplains has advocated for the creation of an official rite for deceased infants. There is no specific funeral service for miscarriage but a funeral service can be arranged by a priest to decrease the parent's distress.

The Sacrament of the Anointing of the Sick, given to the person just before their death, serves as a spiritual cleansing for the ill or dying person (Lewis, 2009). Formal mourning commences with an open casket service, during which a Rosary is recited. Christians are either buried or cremated (Nazarko, 2006). Group prayers are done for the soul of the deceased individual 1 to 2 evenings before the funeral service. During this time family members and friends pay respects to the body of the deceased. A funeral mass is done at the church followed by the procession to the funeral site were a graveside service is performed. The grave is blessed before burial with holy water by a priest or deacon.

Catholic Latino families are most likely to rely on their extended family and church for emotional support but most of the support is given to them by their immediate 
nuclear families. The mourners pay their respects to the deceased by wearing black or dark colors. During the mourning period, the deceased's immediate family members do not watch television, play the radio, or attend social events. The mourning period for older Latino adults may last from months to years. Latino rituals include novenas for 9 days (praying of the Rosary is done each day), mass for the deceased during the first anniversary of their death and lightning candles (Clements et al., 2003; Munet-Vilaro, 1998).

\section{Changes in Religious Beliefs and Practices after the Death of a Sibling}

In an exploratory study by Batten and Oltjenbruns (1999), four adolescents (2 males, ages 17 and 13; 2 females, ages 17 and 16) were interviewed after the sudden death of a teenage brother via a set of open-ended questions regarding: the adolescents own situation; relationship between religion, spirituality, and bereavement; and personal outcomes after their brother's death. Two of the participants were older and two were younger than their deceased brother. The race/ethnicity of the participants was not disclosed. This study clearly depicts the difference between the ways in which adolescents view the death of a sibling compared to younger children due to their elevated levels of cognition. Adolescents reported an ongoing attachment with the deceased sibling which can enhance coping after the loss. Also, some adolescents expressed increased belief in the Higher Power, while others were angry at God after the death of the sibling. For all of the participants, their sibling's death changed their perspectives about life's meaning and served as a catalyst for spiritual development. Results from this small sample are consistent with those from other studies. Longitudinal 
research is needed on death of siblings secondary to expected causes such as chronic illness.

\section{Understanding of Death and Death Rituals}

Children's concept of death. Children today are less aware of the dying process than children in the past because dying has become less public. Willis (2002) states that "American children grow up in a culture that avoids grief and denies the inevitability of death" (p. 221). In the past, individuals died at home surrounded by their family and friends. Now, people are more likely to die in hospitals, hospices, and nursing homes. Children often are not part of the dying process and do not partake in this growth experience which labels them as the first "death free" generation in United States history (Aiken, 2001).

Nagy (1948) conducted a classic study on the meaning of death. The sample was composed of 378 Hungarian children aged 3 to 10 years. All of the children were questioned about their ideas about death. Those who were 6 to 10 years were also asked to make drawings about their ideas with regards to death. Consistent with Piaget's theory of cognitive development, Nagy concluded that there were three stages in the growth of children's understanding of death.

The children in Nagy's first stage (ages 3 to 5) did not differentiate death from separation. They saw death as temporary rather than permanent. Children in this stage don't understand the finality and irreversibility of death. Children may also partake in magical thinking when they believe that they caused the person's death because they wished for the individual to die during an argument. 
The children in Nagy's second stage (ages 5 to 9) saw death as being directly associated with age. They felt that death was something that happened to old people. They saw death as irreversible but avoidable. They believed that only people who are bad die. They tended to accept the mortality of strangers but not the mortality of their loved ones or their own mortality. The children in Nagy's third stage (ages 10 and above) had a more realistic adult-like perspective of death as real, permanent, and unavoidable even if the person is careful. The children in this stage see death as a process that is final and universal.

There are four mechanisms that are associated with children's understanding of death: irreversibility, finality, inevitability, and causality (Willis, 2002). These 4 components have a direct association with the developmental level of the child when the death occurred. The irreversibility factor means that children often do not comprehend that death is irreversible and cannot be altered. Finality explains that death is permanent. Children do not have a realistic concept of time when they are young; hence, it is difficult for them to understand the concept of finality. As they mature they are better able to comprehend the concept of time, and permanence has meaning to them.

Inevitability has to do with the fact that death is part of the natural process of life and it is unavoidable. It is difficult for children to understand this process, especially if they have not had pets (Willis, 2002). Causality is a concept that is difficult for children to understand since they may be unaware that some situations are caused by natural factors that they have no control over. Children who partake in magical thinking may feel that they caused a person's death because they had ill wishes towards the individual. Researchers have different views on children's understanding of death. 
Slaughter and Griffiths (2007) suggest that fundamental ideas of the irreversibility of death begin to form as early as age 5 or 6 . Games such as peek-a-boo which create awareness of object permanence provide a basis for the formation of the concept of death later in life. Psychologists hypothesize that children recognize and comprehend more about death than they are capable of expressing (Aiken, 2001).

Bonoti, Leondari, and Mastora (2013) conducted a study in which children's understanding of the concept of death was investigated through drawings and a questionnaire. The sample consisted of 52 Greek children aged 7, 9, and 11 years. Half of the 52 children had a personal death experience. The researchers did not disclose the participants' religious preferences. Results showed that children's understanding of death is associated with age and past experience. Children who had a personal death experience had a more accurate understanding of death than children with no prior death experience. A limitation in this study (Bonoti et al., 2013) is that cross-cultural comparisons are needed to determine the impact of cultural and religious factors on children's concept of death.

Most of the research on children's concept of death has concentrated on their understanding of its biological aspects such as inevitability, irreversibility, and finality. However, some religions see death as the beginning of a new life. Two studies by Harris (2011), one conducted in Spain and the other in Madagascar, found that as children progress through the stages of development, they enhance their biological notion of death with the religious conception that there is life after death. Harris found that the notion of death as a biological end to life and a non-biological transformation (non-corporeal continuity of the individual's spirit or soul) is not confined to young children or to those 
growing up in a Christian faith. It is also present among adults raised in a non-Christian culture.

Children between 5 and 10 years of age are expected to have limited understanding of the concept of death, while children older than 10 years are assumed to have full understanding of the concept (Willis, 2002). If children fail to understand the finality of death, they may ask when the deceased is coming back. As a result, the bereavement experience of children may be affected by their understanding of the concept of death.

Influence of religion on children's concept of death. Sagara-Rosemeyer and Davies (2007) conducted a qualitative study of 16 Japanese children's views of death in light of their religious traditions. Their view of death and the afterlife encompassed three main religious traditions in Japan (Shintoism, Buddhism, and Confucianism) as well as Christianity. A very strong connection between the children's religion and their notion of death was found. Other investigators have come to the same conclusion as SagaraRosemeyer and Davies (2007). Religious experience is an important variable that may affect children's understanding of death (Bering et al., 2005). The Sagara-Rosemeyer and Davies (2007) study should be replicated with a larger sample and different cultures and religions.

A study done by Yang and Chen (2006) explored Chinese children's concept of death through their drawings. Contrary to other study findings, there was no significant difference in children's death concept by religious belief. The researchers emphasized that in structured interviews and surveys, children's concepts of death were measured with respect to adult standards which may bias the results. They opted for this type of 
analysis because children could openly express their perception about death through their drawings.

Children may rely on their spirituality and religion to cope with the death of a sibling. Pendleton, Cavalli, Pargament and Nasr (2002) found that spirituality is an important component of a child's daily life and that their spirituality may be heightened during times of crisis and ill health. Children's religion has been shown to have a direct impact on children's understanding of death (Bering et al., 2005) which might be of assistance in helping them cope with the death of the sibling.

\section{Experience with Death and Death Rituals}

Throughout history, the deaths of children were not unusual. In countries where mortality rates are high, the death of a child is often seen as inevitable, with mourning lasting no more than a few days (Parkes et al., 1997). On the contrary, in developed and developing countries, changes in sanitary conditions, nutrition and medical advances have markedly decreased the mortality rate in children since the beginning of this century. Childhood deaths are now seen as unnatural and unfair since they do not adhere to the new perceived order of nature.

The death of a child is given unique significance across cultures (Parkes et al., 1997). Each culture is characterized by different beliefs about where children come from before they are born and where they go after they die, which is the same for adults. The child's age, gender, and cause of death will affect the meaning given to such a loss and determine the norms for appropriate grieving behaviors within a particular culture (Parkes et al., 1997). The death of a male child who would carry the family name in future generations and be the provider and protector of the family of origin and family of 
procreation, is seen as more traumatic in certain cultures than that of a female child (Parkes et al., 1997).

Death rituals. Rituals are a combination of metaphors, symbols, and actions in highly condensed dramatic form (Gudmundsdottir \& Chesla, 2006). Reeves (2011) defines a death related ritual as a "ceremony, directly involving at least one person, and the symbols of the loss and, usually directly and indirectly involving others" (p. 409). In the Jewish tradition the Kriah is performed which is the ritual of tearing clothing or wearing a black ribbon to designate that a person is mourning. Members of the Church of Jesus Christ of Latter-Day Saints (LDS) use specific temple clothing to dress the body of the deceased, men dressing men and women dressing women.

Rituals "define the death, the cause of death, the dead person, the relationships of the bereaved with one another and with others, the meaning of life, and major societal values" (Parkes et al., 1997, p. 33). Bolton and Camp (1989) divide death rituals into prefuneral, funeral, and postfuneral. Pang and Lam (2002) state that "through the prefuneral and funeral rituals, the bereaved will come to cognitively accept the death of the beloved" (p. 295). Prefuneral and funeral rituals help the bereaved with the realization that their loved one has died. Gatherings during funeral rituals provide social support to the bereaved. Postfuneral rituals help form continuing bonds between the bereaved and the deceased and this will help the bereaved accommodate after the loss.

Reeves (2011) further separates rituals into preliminal and postliminal. Liminal comes from the Latin word for "threshold". Preliminal rituals are implemented to prepare the dying person or their loved ones for the threshold. An example of this type of ritual is the Catholic Sacrament of the Anointing of the Sick given to the person just before their 
death. The wake, memorial service, and funeral are the most common death related liminal rituals (Reeves, 2011).Whether it is known as a funeral, tribute service, memorial service, or celebration of life of the individual who has died, the purpose of this ritual is to express grief over the death, provide support to the survivors, and prepare to experience life without the one who has died (Weeks, 2004). Postliminal rituals can include scattering the ashes of the deceased on the first anniversary of their death.

Death rituals for deceased children. The rituals performed for wakes, funerals, and burials or cremations for an infant or child are different from those practiced for adults and can differ according to the child's age, gender, and cause of death (Brooten et al., 2015). The death of infants has a distinctive status in most cultures (Parkes, 1997). In some cultures infants are buried exclusively in reserved parts of the cemetery or, as in Japan, may have their own cemeteries and family members may or may not be expected to visit the grave thereafter (Parkes, 1997). In the Asian culture, when an infant or child dies they are profoundly mourned (Lobar, Youngblut, \& Brooten, 2006). The family members dress in white clothing or wear white head bands. The Chinese believe that the death of a child is a "bad death" as is any form of violent death (Parkes, 1997). Parents and grandparents are not expected to attend the child's funeral and the death is not discussed, because a child's death is considered shameful to the family. Family members believe that the gods have not blessed them (Gupta \& Yick, 2002).

For Filipinos, the death of a child is devastating and severe guilt is experienced by the parents (Parry \& Shen Ryan, 1995). Coping is difficult for family and friends. Rituals for deceased children are simpler and shorter to lessen the pain for the family. Filipinos 
believe that children are angels, and since they are innocent and have not committed any sin they go directly to heaven after they die.

An interpretive phenomenological study by Gudmundsdottir and Chesla (2006) describes specific habits and practices created by families bereaved from the sudden and unexpected loss of their children. The data were gathered by interviewing 15 family members in seven US families. The family members were recruited through support groups, advertisements, and bereavement nurses. All of the families in the study consisted of mothers-father dyads with the inclusion of an adult sister from one family. Of the 7 families, five were conventional nuclear families, one step family, and one foster family. The researchers intended to include children in the studies but they faced recruitment difficulties and reluctance from support group facilitators. All of the parents were married at the time of the study.

The participants were 12 Caucasians and 3 Latinos (Gudmundsdottir \& Chesla, 2006). All of the parents had a college education. The household income was high with most of the families earning more than $\$ 80,000$, one family earned between $\$ 40,000$ and $\$ 80,000$, and one family chose not to disclose their income. Family members reported their religion as Protestant (11), Catholic (2), Jewish (1), and Agnostic (1). The children's age at death ranged from 3.5 months to 9 years. Five of the 7 children were 6 months or younger at the time of death. All died suddenly and unexpectedly from different causes: 4 Sudden Infant Death Syndrome (SIDS), 1 histiocytosis, 1 brain aneurysm, and 1 severe gastroenteritis.

Three interviews were conducted with each family (Gudmundsdottir \& Chesla, 2006). Family members were interviewed separately and together. The first and the last 
interviews were conducted with the bereaved parents and children. The middle interview was conducted with the individual family members for them to express their individual feelings and experiences. The first interview took place 6 to 33 months after the death of the child. Interviews lasted for about two hours. The interviews focused on the history of the child's death and the reports of the family's reactions and responses after the child's death.

The families in this study (Gudmundsdottir \& Chesla, 2006) participated in traditional American mourning rituals by having a funeral or a memorial service for their deceased children. Mourning is seen as completed after the funeral or memorial service has taken place. Mourning rituals differ among ethnic and cultural groups in the US. Even though long-term practices related to traditional mourning are emphasized in Chinese, Mexican, and Jewish cultures (Klass \& Goss, 2003; O'Gorman, 1998; Yick \& Gupta, 2002), others do not adhere to these long-term practices. Even though these rituals were important to the families, the support they received through them was transitory, and they continued to suffer from the unexpected death of their children (Gudmundsdottir $\&$ Chesla, 2006). Perhaps this is why some families in this study created rituals to acknowledge their children's death in private and in their own special way.

Some of the families in the study (Gudmundsdottir \& Chesla, 2006) created rituals in which they bonded with their children's spirit. Every time the digital clocks showed equal numbers (for example 1:11,2:22), the mother in one family believed that her child was making her presence known to her and the rest of the family. She told her husband about it and he started noticing the matching digits on the clock and thinking 
about their deceased child. These thoughts of their deceased daughter brought them pleasant memories of her while at home and when they were away on vacation.

This ritualized connection with their deceased child made it possible for the mother, who saw her family as no longer complete, to constantly include her deceased child in the family's life (Gudmundsdottir \& Chesla, 2006). It also provided the father with an opportunity to reminisce and remember his deceased child. Healing rituals created by the families brought solace to them even when they were suffering the most. Mourning rituals have become more diversified and private, personalized by individual families dealing with loss. All of the rituals honored the memory of the deceased child and created an ongoing connection between the surviving family members and the deceased child. Maintaining an ongoing connection with the deceased becomes an integral part of successful adjustment (Field \& Friedrichs, 2004).

A limitation of this study (Gudmundsdottir \& Chesla, 2006) is that most of the families were Protestant and Caucasian. This limits the generalizability of results to families who practice other religions and who are from different cultures. Also, all of the children died suddenly and unexpectedly, and most of them died from SIDS at 6 months of age or younger. These results cannot be generalized to families whose child died from a chronic illness and/or at an older age. Finally, the timing of the first interviews varied greatly from 6 months post-death to almost 3 years post-death. This large range may obscure differences that occur with time.

Differences for deceased children and adults. Anthropologists note that throughout history only abbreviated rites are performed after an infant died and grief is completed almost immediately (Parkes et al., 1997). This is logical due to the high infant 
mortality that existed. Parents expected that not all of their pregnancies would reach completion and that some of their babies would die. This is no longer true.

Latinos and Filipinos believe that deceased children are pure and innocent and that they go directly to heaven after their death. Latinos do not observe the novena for deceased infants as they do for adults because they are pure and have nothing to be forgiven. After reviewing the literature very little information was found regarding specific death rituals performed for deceased children.

Differences by culture/country. Death rituals differ between cultures but they usually share specific psychological and emotional mechanisms intended to assist the survivors to overcome their loved one's loss and deal with the death (Abu-Rabia \& Khalil, 2012). A qualitative study by Lobar et al. (2006) described practices surrounding death of a loved one by Asian, European, Caribbean, Central American, and South American families residing in the US. The sample in this study consisted of 14 female masters nursing students who ranged in age from 25 to 54 years. Five were Black, 4 White non-Hispanic, 3 White Hispanic, and 2 Asian. Five were born in the US, 3 in Jamaica, 1 in Thailand, and 1 in the Philippines. Their religious affiliations were Baptist, Catholic, Jewish, Episcopalian, Hindu, and Buddhist. All of the participants spoke English fluently and many also spoke Spanish. All of the participants were registered nurses with a Bachelor's degree and an average of 14.6 years as a nurse. They were enrolled in the masters nursing track in child health $(n=6)$, adult health $(n=6)$, and family health $(n=2)$.

The students participated in a focus group discussion with the main theme being their personal experiences and practices with death of a family member. A pervasive 
theme was that many similarities as well as differences exist in rituals surrounding the death of a loved one across cultures and religions, making it difficult to separate the influences of culture and religion. Beliefs about the soul of the deceased influenced families to perform rituals and ceremonies believed to foster passage to God, the light, or into another life. The more profound their beliefs were, the more committed the family members are in completing the rituals and ceremonies according to their religion or culture.

Hispanics. Religion and spirituality are of upmost importance for Latinos who practice Catholicism which is the dominant religion for this group (Clements et al., 2003).There is no specific funeral service for miscarriage but a funeral service can be arranged by a priest to decrease the parent's distress. Formal mourning commences with an open casket service, during which a Rosary is recited. Group prayers are done for the soul of the deceased individual 1 to 2 evenings before the funeral service. During this time family members and friends pay respects to the deceased. A funeral mass is done at the church followed by the procession to the funeral site where a graveside service is performed. The grave is blessed before burial with holy water by a priest or deacon.

Latino families are likely to rely on their extended family and church for emotional support but most often, their immediate nuclear families. The mourners pay their respects to the deceased by wearing black or dark colors. During the mourning period, the deceased's immediate family members do not watch television, play the radio, or attend social events. The mourning period for older Latino adults may last from months to years. Latino rituals include novenas for 9 days (praying of the Rosary is done 
each day), mass for the deceased during the first anniversary of their death and lightning candles (Clements et al., 2003; Munet-Vilaro, 1998).

For Mexican-American women, "the wake is the collective sharing of grief and mourning for the dead" (Parry \& Shen Ryan, 1995, p. 112). Family members and friends come to pay their respects to the deceased and offer condolences to the bereaved. Women are the ones who lead the prayers, invite family members to attend, and prepare food and drink for after the wake. The women grieve openly; men do not express their grief or display emotions openly due to the "machismo" tradition.

The funeral is a heart-rending occasion for Mexican-Americans since they feel the presence of death in the family due to the loss of their loved one (Parry \& Shen Ryan, 1995). Family members make every effort to attend the funeral even if they live far away to make their presence known and represent their family ties with the deceased. The family expects to have a large funeral not only to demonstrate how much the individual was esteemed but how strong the family bond is even after death. During the funeral, family members speak of their imminent death in the future and comment on their wishes for a comparable funeral. This type of informal planning is left for a sister or daughter to fulfill when the time comes. The transfer of roles and tasks are passed on from generation to generation in this manner.

During the funeral procession entering the church and cemetery, the matriarch or the eldest women enter first, assisted by other women in the family, followed by the men (Parry \& Shen Ryan, 1995). Women teach the family about funeral customs as the family progresses through the ritual. During the funeral any resentment or ill feelings among family members is set aside since they believe people should be united during this time. 
In the Dominican Republic, family members congregate in a semi-dark room during the novena to pray and to talk about the deceased (Parry \& Shen Ryan, 1995). Individuals also reminisce about other loved ones who have died. People from the community visit the family's home to provide support and come to pay their respects. The rosary is said daily for nine days. This ritual is organized for the mourners to receive emotional and spiritual support from others. The group process involved in the novena can be intense when the rosary is recited. During this nine day ritual, close relatives of the deceased dress in white or black. Red is seen as inappropriate.

It is not unusual for a family member to become "possessed" or have an "attack" (ataque) that may look like an epileptic seizure while remembering and talking about the deceased (Parry \& Shen Ryan, 1995). The ataques are culturally accepted. A mourner possessed by a spirit during these religious activities is within the norm. An ataque is a defense mechanism in which strong feelings felt by the mourner are released in a physical form. When someone experiences an ataque they are in a transient state of partial loss of consciousness, hence, they will manifest convulsive movements, moaning, hyperventilation, profuse salivation, groaning, and some aggressive physical gesticulations. They can commence suddenly and end abruptly.

In some cultures, the children are considered to be pure and innocent and their untimely death guarantees them heavenly status. Puerto Ricans dress their child in white, paint the face to resemble an angel and place flowers outside and inside the coffin (Parkes et al., 1997). Some Latinos believe that the novena done for the adults after their death is not done for infants because they are innocent angels and there is nothing to be 
forgiven (Parry \& Shen Ryan, 1995). This is why deceased infants are dressed in white as a symbol of their religious purity and innocence.

African-American. In the 1900s, it was customary in African American communities to leave the casket open for viewing sometime during the wake and church services. Practices such as laying-on of hands, touching, kissing, and conveying one's grief by viewing the remains are important and have been passed down from generation to generation (Holloway, 2002). This practice stemmed from African funeral traditions in which the family and the deceased were honored with visitations that showed respect and admiration. In the US the tradition also may symbolize a different kind of honoring, since it recognizes specific evidence of the violence done to Black bodies secondary to racism (Parkes et al., 1997). For example, after Emmett Till's lynching in 1955, Till's mother insisted on an open casket in order for others to see what had been done to her child. Thousands congregated in the South Side Chicago streets to view his remains.

The significance of involving the community in the African American funeral ceremony is from West African cultures that saw the death and burial as an important, public, elaborate, and lengthy social event (Holloway, 2002). Also, in Black funerals, there is strong, loud and unrelieved weeping and wailing. There are nurses, the church deaconesses or church mothers dressed in white (sometimes black), who stand near the bereaved family with tissues and fans, ready to aide a mourner or ease the fall of the faint. African American funerals are longer than those of White Americans.

The traditions of West Africa, the mourning and dancing, were strongest in twentieth century Black America in the jazz funerals of New Orleans with the performance of music from legendary brass bands (Holloway, 2002). The Kongolese 
slaves who had been brought to Louisiana would express the soul's sorrow with customary weeping and wailing before they accompanied the dead to the burial sites with much jubilation. It is customary for the mourners to sing, beat the drum, and dance the soul to its new home.

In African American communities in the US, the wake is the first occasion in which comfort and assistance is given to the bereaved families (Holloway, 2002). The food served during this time is as essential as the conversations and recollections that family and friends bring with them to the family home. Throughout the beginning of the nineteenth century the amount of food and the attention given to its preparation were important and seen as signs of respect to the bereaved family. Bringing prepared food from the store was looked down upon by the African American culture. Fried chicken has been a staple during these occasions. But, in the last quarter of the $20^{\text {th }}$ century with the introduction of fast foods, specifically fried chicken outlets, food preparation by the family has been replaced by a bucket from Kentucky Fried Chicken (Holloway, 2002). The tradition of providing meals to the grieving family starts with the wake and concludes with a formal meal after the funeral service.

Caribbean Black. Throughout history, Christian and indigenous (Arawak, Carib, African) components in the Caribbean Black culture have been almost inseparable (Kerns, 1997). Black Caribs have been affected by Roman Catholicism for over 250 years as well as Amerindian and African influences. The Black Carib settlements are located in Belize. Most labor migrants have traveled to the US since the 1950's. A large number of Belizeans, especially, Black Caribs and Creoles, now live in several North American cities. 
Rituals for the dead are done to take care of their needs and protect the living from illness, misfortune, and even death brought upon by a resentful spirit (Kerns, 1997). Shamans, who are mostly Roman Catholic, conduct some of the rites as well as the older women who help organize most of the rituals. Black Caribs know that some of their beliefs and rituals as well as their language, dance, and music are different and this distinguishes them from other races (Kerns, 1997).

Many of their beliefs and rituals stem from the Catholic religion; hence, they see no conflict between Catholic dogma and their own beliefs (Kerns, 1997). They often explain their own beliefs in terms of their understanding of Catholic orthodox theology. Black Caribs consider ritual offerings presented to spirits of lineal kin to be the same as prayers said for the souls of the deceased at a church. The ceremonies for the deceased have a festive quality to them and Black Caribs call them fiestas. Dancing and singing are part of the ceremonies for the deceased. All of the rituals include cooperative work and the distribution of food. Sharing food with the living, and through offerings, with the dead is a fundamental ritual that symbolizes trust and kinship, kindness to the living, and gratitude to the dead. Most of the rituals are done at dawn or dusk, or continue from dusk until dawn.

Preparations for the wake begin immediately after the death by women who are close relatives of the deceased and by other women who have volunteered to help (Kerns, 1997). Before the burial takes place the next day, the body must be washed and dressed usually by older women. In some cases the funeral garments have been prepared beforehand by the deceased. The funeral garment for men consists of a black suit and white shirt and for women, a long white dress. The men usually construct the wooden 
coffin while the women prepare refreshments. Coconuts, flour, and other ingredients used to make bread are obtained, as well as rice or corn to be roasted and boiled for gafe, a black coffee-like drink sweetened heavily with sugar. The women will offer this food throughout the night to family and friends of the deceased who attend the wake.

Depending on how much food they have, they may distribute food to nearly every person in the community who comes to view the body.

Wakes are serene early in the night (Kerns, 1997). The men talk with each other or gamble while some of the women sing hymns or talk with each other quietly while distributing food and rum. As more rum is passed around, the ambience changes and the crowd becomes more animated and sociable later in the night. Eventually drums are used to provide music for dancing. Family members of the deceased drink liberally during the wake and before the burial to help them bear the grief. Individuals use rum to wash their hands after they have prepared the body for burial.

Close relatives of the deceased arrive throughout the night and during the morning before the burial (Kerns, 1997), usually more women than men. As each woman arrives she begins to wail praising the deceased and expressing her gratitude for the support and care that the person had provided for her. Wailing is a ritual skill that women are encouraged to develop. It is rare for men to wail. Men will approach the coffin and say a few words of gratitude to the deceased speaking in a somewhat emotionless manner.

A novena for the deceased, part of the Roman Catholic tradition, begins on the first or second Friday after burial (Kerns, 1997) to assist the departure of the soul from the physical world. The ninth-night wake is held at the conclusion of these prayers and serves the same purpose. During the ninth-night wake, women will serve bread, coffee, 
sweet rice, and conch soup to those who have participated in the novena. At about 8:00 p.m. prayers will begin inside the house with a dozen or so older women. After the prayers end, the ambience is characterized by music and dancing, until 5:00 a.m. when some of the women gather again to pray. Men usually do not participate in the prayers at night.

Close female relatives of the deceased do not sing or dance (Kerns, 1997), mourning for a period of six to 12 months after the death, depending on their relationship to the deceased. Men and children do not mourn formally. They can continue to work and socialize. On the contrary, women can attend social events only as observers. They may not dance or drink.

Women who are mourning do not dress entirely in black (Kerns, 1997). They wear "second mourning dress" which includes a print of black and white, dark blue and white or other subdued color. They do not wear earrings or any other jewelry during this period. A widow does not work for two months after her husband dies and is not supposed to wash or cook. During this time the bereaved woman is assisted by other women who help her with the household tasks and keep her company even while she sleeps. Tradition states that the spirit of the dead might bother her if she is left alone. The bereaved woman needs to stay inside the house for two weeks and she is not supposed to leave the house after dark for several months. For women who are mourning a parent or spouse, a ceremony is held one year after burial to symbolize the conclusion of these observances.

During this ceremony the mourning women go to church to pray (Kerns, 1997). Anyone can attend but mostly the older women are part of this ritual. After the prayers 
conclude women go to the beach to watch the mourning women perform a bath ritual. Each of the mourning women enters the beach fully dressed with their arms linked to a female partner, who is usually an aunt or an elder cousin. The companions submerge the women and then they help them up repeating this twice again. After this, they leave the beach and walk inside the house. The women tear the back of the black dress from each of the mourning women's bodies. The mourning women remove their underclothing and enter another room to dress, protected from view by other women who have congregated around the doorway and stairs.

In the back room the mourning women get dressed in bright colored clothing and head wraps, and they don earrings and other pieces of jewelry (Kerns, 1997). In the corner of the room a small plate of food and a bottle of rum are placed as an offering to the spirit of the deceased. Above the offering of food and drink, the suit of the deceased man's or woman's clothing is hung. The women drink rum and eat bread and coffee. The mood is festive with songs of remembrance. This ritual is only done in Black Carib communities.

If a close relative has a dream in which the deceased requests a bath, the Amuidahani ceremony will take place (Kerns, 1997) during dawn in the yard of the house were the deceased lived. This ceremony takes place usually within a year after the death. A shallow pit the size of a grave is dug and close relatives of the deceased will come together around it for the brief ceremony. Each of the individuals present will through a bucket of water in the pit. They will do so in pairs with one person standing at the head of the pit and the other at the foot with the closest relatives throwing the first buckets of water. After everyone has taken a turn, the pit is covered. The women begin to sing, 
dance punta (traditional dance) and distribute food (bread) and drinks (rum and coffee) to the guests. This ceremony can be curative in nature. If any close relative has been suffering from an ailment caused by the spirit, after Amuidahani, the symptoms often disappear.

A Requiem Mass is also done if the spirit requests it in a dream usually to a close relative (Kerns, 1997). The mass is usually done at church. Prayers are said. After the mass ends, all the people will go to the house of the woman who sponsored the mass. Bread and coffee are distributed and the women sing remembrance songs. An offering of food and rum is set aside for the deceased alongside their clothing and a burning candle.

The ritual of Chugu (feeding the dead) and Dugu (feasting the dead) are obligatory when requested by a deceased family member through dreams (Kerns, 1997). Chugu consists of an elaborate offering with frequent prayers led by a shaman as well as singing. Food is offered in the house of the deceased but most do not eat because they fear becoming ill if they eat the foods that the spirits have tasted. Chugu does not involve dancing or drums which are part of Dugu. Dugu is costly, elaborate and lengthy. The purpose of Dugu is to bring peace to an ancestor who has caused distress to one or more family members. A year of planning is often needed to make this ceremony possible. This ritual is held in the temple and consists of three or more days of drinking, dancing, and dining. During this ritual a hog and chickens are sacrificially slaughtered and their remains are displayed. The central ritual act of Dugu is the dance of placation done to appease the spirits. The older women have a central role in all aspects of the Black Caribbean rituals. 
Jamaican beliefs about death and dying stem from a mixture of African and European religious and cultural traditions (Burrell, 1996). In Jamaica a death ritual by the name of "nine night" is done, which starts from the date of death and ends nine nights later. This is a ceremony of passage where people congregate to provide support to the relatives of the deceased and to wish the deceased a safe journey to the next phase in life. Jamaicans believe that the spirit of the dead can cause harm.

Song is fundamental in the nine night ceremony (Burrell, 1996). Singing starts three days and nine nights after the death. The night before the burial, a wake is done and 40 days after the death another singing takes place. This rite of passage serves to appease the spirit of the deceased which roams for 40 days and 40 nights before finally resting. The nine night ceremony is held under a tent built next to the house. A table covered with a white cloth is positioned in the center of the tent and fried fish and cassava bammy (cake) or white bread will be placed on it. The food is left on the table until midnight when the spirit of the deceased is said to pass through and has something to eat. Rum and dancing are important.

Raising and lowering the coffin is done three times, to inform the earth goddess Asase that the new spirit is about to join the others (Burrell, 1996). The deceased's coffin is transported by hearse from the funeral parlor to their home or to the cemetery (Burrell, 1996). If the body is returned to the home and infants are present, they are crossed over the coffin twice so that the spirit of the deceased does not lure away their spirits. "Journey cakes", also known as johnnycakes, may be placed in the coffin for the deceased to have food to eat during their journey to heaven. If the deceased had lived a life characterized by violence, spiritual healers conduct an exorcism in which the spirit of 
the dead is "tied" and buried under a cotton tree. It is also customary for relatives to seek assistance from obeah or voodoo practitioners to perform rituals and make contact with the deceased's spirit.

Native Americans. Native American tradition, beliefs, and ceremonies vary widely among Native American groups (Clements et al., 2003). The culture of each reflects the influences of assimilation, acculturation, relocation, and education at boarding schools. Native Americans currently practice several Christian religions such as Catholicism, Jehovah Witness, and Presbyterian (Shaefer, 1999). In all tribes the elders are revered and their wisdom and experiences are respected.

Native American mourning practices are affected by the degree and nature of family support and the status of the deceased in the community (Clements et al., 2003). Navajo is the second largest Native American population in the United States. The tribe is divided into more than 50 clans. The Navajo believe that death is another phase in an individual's life and that traditional rituals for burial and mourning must be performed to ensure their transition to the next world. Rituals not performed accordingly will affect the well-being of the surviving relatives and the spirit of the deceased.

The deceased's family members and friends have 4 days after the death to cleanse and prepare the body for burial, mourning, and disposing of the deceased's belongings (Clements et al., 2003). Cleansing of the body is the initial step in preparing the deceased for the journey. The body is washed, and the face is painted with chei (a war paint made of a soft red rock crushed and blended with sheep fat) and white corn to safeguard the deceased on the journey. The deceased will be dressed in his or her best clothing and 
blessed with corn pollen, and the hair will be tied with an eagle feather to symbolize a return home.

The deceased's body is buried in the family's hogan (a sacred home) and then the hogan is abandoned (Clements et al., 2003). Every Navajo family must have a hogan for ceremonies and to maintain spiritual balance even though they live in a newer home most of the time. All of the deceased's possessions are disposed of by burying them with the body, giving them away to others or burning them.

On the morning of the fourth day, the deceased's family members wash themselves on the eastern side of the home as a symbol of cleansing themselves after the burial ritual (Clements et al., 2003). The cleansing ritual allows the deceased's spirit to start the journey north to his or her next life. After the cleansing ritual the Navajo believe that any conversation about the deceased, even years later, will prevent his or her spirit from continuing the journey. Speaking of the deceased, even years after the death, could physically and mentally affect the surviving relatives and their families.

Europeans. Compared to other industrialized nations, US society is strikingly heterogeneous with differences in how and under what circumstances individuals die (Fontana \& Keene, 2009). Funeral rituals in the 1600s consisted of a sermon in the church, followed by a procession walking to the gravesite with pallbearers carrying the coffin. At the gravesite, the people remained silent as the casket was covered with dirt. Afterwards there was a celebration with large quantities of food and liquor. Americans in the US continue to prefer traditional funerals which include embalming, open casket viewing, and elaborate caskets (Fontana \& Keene, 2009). 
In a study by Fristad, Cerel, Goldman Weller, and Weller (2000), 318 parentbereaved children aged 5-17 (98\% White) and their surviving parent were recruited from the community and interviewed at 1, 6, 13, and 25 months post parental-death about their participation in funeral related rituals and adjustment after the death. Fifty-nine percent of the participants were children (5-12 years old) and 41\% were adolescents (13-17 years old); $50 \%$ were male.

One to four children per family participated (Fristad et al., 2000). The families' socioeconomic status ranged from upper to lower class, and averaged middle class. Children and parents were interviewed separately and simultaneously by different interviewers. The initial interviews lasted four hours and follow-up interviews, one to three hours.

The instruments used in the study were The Funeral Questionnaire-Child and Parent forms (Weller, Weller, \& Fristad, 1984a), The Grief Interview-Child and Parent Forms (Weller, Weller, \& Fristad, 1984b), The Child's Depression Rating Scale-Revised (Poznaski, et al., 1984), Diagnostic Interview for Depression in Children and Adolescents (Weller \& Weller, 1979), and The Diagnostic Interview for Children and Adolescents (Reich \& Welner, 1988).

Almost all of the children attended their parent's visitation, funeral, and burial. About half (54\%) of the parental deaths were unanticipated (Fristad et al., 2000). Most families (89\%) had a visitation (Fristad et al., 2000). Nearly all children (96\%) whose families had a visitation attended. The families' definitions of visitation consisted of prefuneral gatherings at the funeral home and post-funeral gatherings in families' homes (wakes or shivas). Of the 34 children who answered open-ended questions regarding their 
reaction to the visitation, $43 \%$ reported that they mostly liked certain aspects of the rituals, including the use of music, prayers, flowers, and placing something in the casket, such as a picture, letter, or holy water. Children also commented on how they liked the support of others (17\%), the eulogy (11\%), and the appearance of the deceased (4\%). Parental responses were similar. More than half (52\%) of the 21 parents who responded to this open-ended question also reported that their children enjoyed the ritual aspect of the visitation.

Almost all of the families (98\%) had funerals and (97\%) of the children attended (Fristad et al., 2000). The children reported (30\%) that they liked positive rituals such as prayers, flowers, and music, such as the deceased's favorite song. Seventeen percent of children mentioned that they liked friends composing music, being able to express their feelings, and picking out the deceased's clothes. Of the 43 children who responded to what they did not like about the funeral, $32 \%$ commented that they did not like the behavior of others (family and friends).

The majority of families ( $88 \%$ ) had a burial service and $97 \%$ of the children attended (Fristad et al., 2000). In 10\% of the families without a burial the deceased was cremated. Of the 52 children who answered what they like at the burial, $33 \%$ reported that they most liked specific aspects like laying a flower on the casket. Twenty-one percent of the children reported that attending the burial helped them with closure and accepting the permanency of the death. One third of the 39 children answered that the lowering of the casket was what they liked least.

The majority of the children reported that music, prayer, and the support of others helped them during this difficult period of time (Fristad et al., 2000). Many children also 
reported that active participation (choosing flowers) held a positive value for them. Children who reported that they acted in an unusual way at the visitation, funeral, or burial were more likely to experience psychiatric symptomatology 6 to 25 months later. Visitation or funeral behaviors such as laughing, hiding, running away, and acting very upset were more highly associated with post-traumatic stress disorder (PTSD) and depressive symptomatology at 13 and 25 months than being more quiet and withdrawn as usual. On the contrary, children who reported being more quiet at the burial experienced more PTSD symptoms at 25 months than those who acted out. It could be that those children that were quieter were able to take in more of the experience than those which exhibited more externalizing behaviors.

Children whose deceased parents were cremated did not differ from other parentbereaved children initially or in the first two years post-parental death (Fristad et al., 2000). Families that had the deceased cremated had rituals after the death which served as an outlet for the family members to grieve and express their feelings with their friends and relatives. Children who did not participate in visitation after their parents' cremation did not do as well one to two years post-parental death as the children that had participated. Limitations in this study (Fristad et al., 2000) consist of the sample being largely composed of individuals who were Caucasian and Protestant. It is not known whether these results apply to children from different cultures and religions.

Asians. The Chinese culture and customs have remained vibrant for the past four millennia and their culture and traditions are carried on wherever they live (Hsu et al., 2009). Buddhism, Taoism, and Confucianism are the three main religions in Chinese culture. Chinese families feel that discussing issues about death is a taboo because it can 
bring bad luck (Chan \& Mak, 2000), and they will try to extend the patient's life as long as possible, while understanding that death is part of the cycle of life. Chinese believe that the spirits of the dead continue to remain on earth, and the ancestors' unhappiness will bring back luck to the immediate and extended family.

In the Chinese culture, the patient is brought home from the hospital when death is expected and moved into the main hall of the house, which is the holiest place of the house and where the ancestral tablets are located (Hsu et al., 2009). Chinese people believe that an individual who dies in the main hall will reunite with his/her ancestors represented by these tablets or the family altar (Jochim, 1986). Relatives who are older than the deceased are encouraged not to attend the funeral.

Other Chinese death rituals include mourning rituals on every seventh day for forty-nine days and on the hundredth day, chanting prayers to help the deceased reincarnate, contacting the dead through a shaman, making offerings to the dead, and rituals of continuity (Pang et al., 2002). Worshipping the deceased at home or contacting the deceased through a shaman enables a spiritual connection between the bereaved and the deceased. Chinese mourning rituals currently last 49 days (Gupta \& Yick, 2002). During this time of mourning, families place black bands of cloth on their sleeves and women are expected to wear a flower made of wool in their hair (Lee, 1991). Family members congregate at the gravesite and burn money and food on the third day after the funeral and the $21^{\text {st }}, 35^{\text {th }}$, and $49^{\text {th }}$ day after the death (Tanner, 1995). The connection between Chinese ancestors and their descendants is a major aspect in Chinese death rituals. 


\section{Response to Death of Parent, Grandparent, or Sibling Conceptual definitions of grief and bereavement}

Bereavement (defined as having a loved one die) and grief (defined as the reaction to the death of a loved one) are two concepts not easily understood during childhood and adolescence, and only few longitudinal studies have been reported (Wilkins \& Woodgate, 2005; Woodgate, 2006). Most of the studies pertaining to child and adolescent bereavement have concentrated on the death of a parent, not a grandparent or sibling (Sood et al., 2006).

One of the most stressful events that a child can experience is the death of a parent (Harrison \& Harrington, 2001). Retrospective and prospective studies have demonstrated that parentally bereaved youth are at risk for psychological problems which include depression, posttraumatic stress disorder, substance abuse, and health risk behaviors (Brent, Melhelm, Donohoe, \& Walker, 2009; Maier \& Lachman, 2000). In studies of adults who had lost a parent as a child, the death of a parent was associated with depressive symptoms, poorer health outcomes, lower sense of mastery and selfconfidence, less perceived family support, and diminished educational attainment (Mack, 2001; Maier \& Lachman, 2000; Marks, Jun, \& Song, 2007). The death of a parent is a traumatic event for the child that leaves lasting negative repercussions.

The death of a grandparent has been documented as the most frequent type of bereavement among adolescents (Westerink \& Stroebe, 2012). Special bonds between adolescents and grandparents can exist due to a significant degree of grandparent caregiving (Milan \& Hamm, 2003). Over time families have become smaller and the number of working mothers has increased, hence, many of the caregivers are 
grandparents and it is logical for children to form such a strong bond with their grandparents. The grandparent-grandchild relationship can be based on unconditional, mutual love which is unique compared to the parent-child relationship (Westerink \& Stroebe, 2012). There is limited research on the consequences that the death of a grandparent has on the child.

The surviving child's developmental stage of life when they experience a sibling's death interacts with the grief process and may pose additional challenges for the surviving child (Lohan \& Murphy, 2001). In the relationships characterized by rivalry, the surviving children may experience guilt after the death of the sibling, and anger for having to feel guilty, specifically since the parents' focus of attention is the dead sibling (Crehan, 2004). The child may feel guilty after the sibling dies if they had previously wished that the sibling would die or go away. Lack of information regarding the death may elicit magical thinking which can create more terrifying and painful interpretations of the event (Crehan, 2004). Children may believe that the sibling died because they harbored negative thoughts or fantasies toward them. They may feel confused and scared that they caused the death because they wished it upon the sibling.

Research with bereaved children and adults finds those deaths which were more recent (within 1-1.5 years), unexpected, in the hospital versus the home, and associated with prolonged illness and/or suffering may be associated with more grief reactions and distress (Barry, Kasl, \& Prigerson, 2002; Gamino, Sewell, \& Easterling, 2000; Seecharan, Andresen, Norris, \& Toce, 2004).

\section{Behavioral manifestation of children's grief after the death of a parent,} grandparent or sibling. Uncomplicated bereavement is the term used in the Diagnostic 
and Statistical Manual of Mental Disorders (DSM-4) (APA, 2000) to define the classic grieving process in which individuals coping with the death of a loved one report symptoms such as intense sadness, sleep and appetite problems, lack of interest in regular activities, and difficulty concentrating. Oltjenbruns (2001) poses that "childhood development is ongoing; grief and feelings of loss are often repeatedly processed as children become more capable of understanding their loss" (p. 73). Children manifest their grief differently, and duration of their grief responses may be underestimated by adults. Loss in childhood should be examined in the context of a child's cognitive, emotional, and social development (Paris et al., 2009).

Willis (2002) explains that children sometimes demonstrate unacceptable behaviors as an expression of their confusion after the death of a loved one. Children are less likely to accept death and tend to grieve intermittently for several years (Aiken, 2001). Grief can be prolonged and can resurface throughout the child's life when they examine the death from their different and maturing developmental perspective.

Christ (2000) captured grief responses from 157 children (age 3 to 17 years) from 88 families after the death of a parent from cancer. The families had participated in a parent guidance preventive intervention. The goal of the intervention was to assist the children by providing the parent with education and support that would help them to: provide support and care for the children, provide an environment in which the children were able to express their feelings and fantasies about the loss, and maintain consistency in the children's environment. The intervention lasted for 14 months and included an average of twelve 60- to 90- minute interviews. The sequence of interviews included an initial interview with the parent(s), then an interview with each child, then an informing 
interview with the parent(s), then a family interview, and then two or more biweekly to monthly parent interviews. A similar schedule was followed starting 2 to 4 weeks after the death of the parent were the interviewer maintained telephone contact with the surviving parent until 14 months after the death.

Children with psychiatric disturbances were excluded (Christ, 2000). The majority of the parents ( $\mathrm{n}=97)$ were Catholic, had a college education $(\mathrm{n}=104)$, and were White ( $\mathrm{n}=80)$. Children's responses to the parents' deaths varied across age groups likely due to the impact of development on children's expression of grief. For children 3 to 5 years old their mourning was characterized by regressive behaviors. For the 6-to 8-yearold children, they understood the permanence of their parents' death once they were informed. Their mourning was different. They submerged themselves in joyful memories of their deceased parents. The 9- to 11-year-old children were not tolerant of strong emotions in themselves and in others, and they participated in activities when they experienced short bursts of grief. The 12- to 14-year-olds were not receptive to information regarding their parents' death since this threatened their defense of denial. They were also intolerant of strong emotions in themselves. Adolescent grief responses (15 to 17 years) varied when compared to the other groups of children in that adolescent grief responses were as intense as that of their parents but not as prolonged. They described intense longing, sadness, helplessness, despair, and hopelessness that prevented their normal functioning in activities such as school, sports, and after-school activities. Researchers have observed that adolescents appear detached and avoid talking about the deceased in order to regulate powerful emotions (Packman, et al. 2006). Adolescence is a difficult period in child development to grieve the death of a sibling since important 
physiological, relational, cognitive, and psychosocial change take place during this time (Lohan \& Murphy, 2001). Differences in grief expression were found across age groups and stages of development.

The study's (Christ, 2000) sample was middle class and only included families that participated in the parent guidance preventive intervention. This study should be replicated with participants from other religions and cultures as well as with more at-risk populations (low economic status). Also, longer term follow-up studies of bereaved children are needed to determine if grieving responses in children change over time.

A longitudinal study by Brent, Melhelm, Masten, Porta, and Walker-Payne (2012) evaluated the impact of sudden parental bereavement on subsequent attainment of developmental competencies (education, work, and interpersonal relationships). The sample for this study consisted of 126 youth bereaved by sudden parental death (suicide (34.6\%), accident $(23.7 \%)$, or natural death $(41.7 \%)$ ) assessed at 9, 21, 33, and 62 months after parental death and 116 demographically similar non-bereaved controls assessed at comparable times. Half of the participants were female and most of them were Caucasian (84.7\%). Bereaved families were recruited through coroner's records $(49.7 \%)$ or by newspaper advertisement (50.3\%). Control families were recruited using random-digit dialing and by advertisement. Bereaved children and children in the control group had to be 18 years or younger.

Participants' developmental competence was evaluated using an adaptation of the Status Questionnaire, peer attachment using the Inventory of Parent and Peer Attachment, and educational aspirations using the Future Expectations Scale. Bereaved youth had lower attachment, more difficulties at work, decreased educational aspirations, and less 
elaborated plans for career attainment. Children who had lost a parent to sudden death had decreased work competence, peer relations, educational aspirations, and career planning.

A study by Gray, Weller, Fristad, and Weller (2011) examined depressive symptoms in bereaved children and adolescents two months after the death of a parent. The sample consisted of 325 children and adolescents age 5 to 18 years recruited from obituaries and funeral homes. They were compared to 129 non-bereaved community controls (recruited from schools, churches, and community groups) and 100 nonbereaved depressed controls (recruited from chart reviews of inpatients and outpatients at a university medical center).

The Diagnostic Interview for Children and Adolescents Revised was used to assess depression. The Children's Depression Inventory and the Children's Depression Rating Scale Revised were used to measure severity of depression in the children. At two months post-parental death bereaved children were more likely to have a major depressive episode compared to community controls (25\% vs. $1 \%, \mathrm{p}<.001)$ but were less likely to have a major depressive episode compared to depressed controls $(76 \%, \mathrm{p}<.001)$. It can be that less severe depressive symptoms by recently bereaved children are in the range of normal (non-pathologic) after a major stressor such as parental death.

A study by Brent, Melhem, Donohoe, and Walker (2009) examined the effects of bereavement after a parent's death. The participants consisted of 176 offspring ages 7 to 25 who's parent had died secondary to an accident (31), sudden natural death (52), or suicide (37). They were assessed at 9 and 21 months after the death alongside a control group of 198 non-bereaved subjects. The bereaved families were recruited through 
coroner's reports and newspaper advertisements. The control group participants were recruited by random-digit dialing or by newspaper advertisement.

Severity of depression, anxiety, post-traumatic stress disorder, and suicidal ideation were assessed in the children by using the Mood and Feelings Questionnaire, the Screen for Child Anxiety-Related Emotional Disorders, the child Post Traumatic Stress Disorder Symptom Scale Interview, and the Suicidal Ideation Questionnaire-JR. Equivalent measures in adults were obtained by using the Beck Depression Inventory, the Beck Anxiety Inventory, the Post Traumatic Stress Disorder Symptom Scale Interview, and the Suicidal Ideation Questionnaire.

Alcohol or substance abuse and major depression were more common among bereaved youth than among the control group 21 months after the parent's death. Offspring of parents who died by suicide and accidents had higher rated of major depression than the comparison subjects, and the offspring of suicide victims had a higher rate of alcohol or substance abuse disorders than did the comparison subjects. The majority of the participants in this study were Caucasian and death by homicide was not included limiting the generalizability of the results.

A study conducted by Ens and Bond (2005) examined the relationships between bereavement following the death of a grandparent and adolescent death anxiety using Hogan's Inventory of Bereavement and the revised Death Anxiety Scale within private schools. Adolescents (226) attending private schools in Manitoba, Canada completed a response to the question "In your family, have your grandparent(s), parent(s), brother(s), sister(s) died?" The most frequently reported death was that of a grandparent (66 grandmothers and 117 grandfathers). The student's ranged in age from 11 to 18 years. 
Since a small number of respondents reported a death of someone other than their grandparent their data was eliminated from the analysis.

Participants completed the pen and paper questionnaires during school hours (Ens \& Bond, 2005) examined. Death anxiety was measured using the revised Death Anxiety Scale (RDAS) and grief and personal growth were measured using Hogan's Inventory of Bereavement. Grief due to bereavement was found to be significantly associated with death anxiety. This study did not explore the quality of the grandparent-adolescent relationship. Death anxiety in the adolescent may vary depending on the degree of attachment to the grandparent. Also, the study did not identify if the deaths of the grandparents were expected or unexpected which may also have a different effect on grief and bereavement in the adolescent. There is a scarcity of research on child and adolescent grief reactions after the death of a grandparent.

Research in sibling bereavement has found an array of grieving responses: emotional (depression, sadness, anxiety, feelings of guilt), behavioral (sleep disturbances, acting out), social (withdrawal, loneliness), and deterioration in school performance (Fanos et al., 2009). Bereaved children may not find the emotional support they need from their parents who are focused on their own pain (deCinque et al., 2006), and some children may not seek support or talk about their reactions thinking that they have to protect their grieving parents (Dyregrov \& Dyregrov, 2005).

A study conducted by Gerhardt et al. (2012) compared peer relationships among 105 bereaved children, ages 8 to 17 years, after the death of a sibling and examined gender, grade level, and time since death as moderators. The majority of the children were female $(\mathrm{n}=57)$ and White $(\mathrm{n}=85)$ with an average age of 12.79 years $(\mathrm{SD}=2.56)$. 
Deceased siblings averaged 11.8 years of age $(\mathrm{SD}=5.17)$ at the time of death. The researchers did not specify whether the deceased siblings were older or younger than the surviving children. Causes of death were cancer diagnoses: lymphomas $(n=10)$, leukemias $(n=23)$, brain tumors $(n=26)$, and other solid tumors $(n=44)$. Three classmates of each bereaved child were matched for gender, race, and age to create a comparison $\operatorname{group}(n=311)$.

The teachers reported on children's social behavior (Gerhardt et al., 2012). Teachers described bereaved children as more pro-social than their comparison classmates. Also, teachers and peers reported bereaved children in middle and high school to be higher in leadership and popularity. Teachers may have been more perceptive of positive changes in children.

Peers in this study perceived bereaved boys as more sensitive-isolated and more victimized (Gerhardt et al., 2012). In contrast, research with parentally-bereaved children (Schmiege, Khoo, Sandler, Ayers, \& Wolchik, 2006) and other studies have indicated that girls are at a greater risk for difficulties than boys when a sibling dies (Sandler, Ma, Tein, Ayers, Wolchik, Kennedy, \& Millsap, 2010). Bereaved children in elementary grades were perceived by peers as less pro-social, more sensitive-isolated, less accepted, and as having fewer friends (Gerhardt et al, 2012). Younger children may be more socially vulnerable because their parents are grieving and they may not be emotionally available for them when they need them the most (Packman et al., 2006) and they have fewer other choices than adolescents who are more autonomous and used to confiding in friends (Barrera et al., 2013). Most of the sample in this study was White (81\%) 
(Gerhardt et al., 2012). The siblings all died of cancer; hence, findings may differ for children who experience a sudden sibling death.

Barrera et al. (2013) conducted a longitudinal study that investigated parental perceptions of children's bereavement and grieving after the death of a sibling due to cancer. The sample consisted of 20 bereaved families (18 mothers and 13 fathers). The mother was the primary caregiver for the deceased sibling and the surviving children in $98 \%$ of the cases. At 6 months (Time 1) there were 15 older siblings (> 10 years) and 8 younger siblings ( $<9$ years) versus at 18 months post death (Time 2 ) were there were 7 older siblings and 7 younger siblings. The majority of the parents were White (74\%) and had completed college or university (65\%). The parents were interviewed at Time 1 and at Time 2 using an interview guide with open-ended questions.

The expressions of grief reported by parents of children who were younger than six years of age consisted of sadness (crying), anger, and behavioral problems (temper tantrums) because they no longer had the deceased sibling as a playmate (Barrera et al., 2013). At Time 1 two mothers who had young children (aged 5 and 8 years old) communicated that the surviving child was having difficulty understanding that the deceased sibling was not coming back, and at Time 2, they described the surviving child as having intense grieving, crying more, and having nightmares about the deceased sibling, suggesting that the surviving child had become cognizant that the deceased sibling was permanently gone. Parental reports suggest that the surviving children below 6 years of age may understand the permanence of death earlier than children their age who have not experienced the death of a brother or sister. These findings conflict with Sood et al. (2006) who found a realistic understanding of the concept of death beginning 
at 10 years of age. From the first interviews, parents were aware of the developmental differences in the surviving children's needs, expression of grief, and what helped them (Barrera et al., 2013).

Themes reported by adolescents included difficulty concentrating in school, not revealing their feelings of sadness to their parents, and engaging in risk behaviors such as tattoos, drinking, and body piercing (Barrera et al., 2013). The parents noticed that adolescents' grief reactions were intense soon after the death but became less evident to parents by Time 2 . This outcome may be related to bereaved adolescents' propensity to talk less with parents than with friends (Hogan \& DeSantis, 1994).

Only three mothers $(20 \%)$ described their surviving child's expression of grief both at Time 1 and Time 2 (Barrera et al., 2013). Parents were grieving over the deceased sibling which might have hindered their perception of the surviving children's expression of grief. This could be why parents' reports on children's grieving and coping are short and superficial. It would be helpful to examine children's expressions of grief through self-report and compare these findings to the parents' perceptions of grief in bereaved children. The sample was markedly reduced at Time 2 (from 25 to 15 parents) and there were fewer fathers compared to mothers across time which may have prevented saturation of themes among fathers and affected comparative analysis of mothers' and fathers' perceptions. Also, the majority of the sample was White and had a college degree. The study (Barrera et al., 2013) should be replicated with children that have died from other chronic illnesses, or other causes (sudden death secondary to suicide or accident). 
A study by Foster et al. (2012) examined changes in children after the death of a brother or sister from the mother's, father's, and child's perspectives within the first year post-death. The sample consisted of 24 fathers, 36 mothers and 39 children from 40 families. Children were primarily female (64\%) and White (78\%), and had an average age of $12.3(\mathrm{SD}=2.64)$ years. Deceased siblings were $54 \%$ male, ranged in age from 8 to 17 years old, with an average of $2.7(\mathrm{SD}=2.34)$ years from diagnosis of cancer to death. Semi-structured interviews using open-ended questions were conducted with each parent and child separately in the home.

Children were more likely to report positive changes that reflected growth and adaptation such as greater maturity $(15 \%)$ and greater compassion (23\%) than fathers (maturity: 1 father; compassion: $8 \%$ ) and mothers (maturity: 22\%; compassion: 8\%) (Foster et al., 2012). Parents were more likely to report negative changes in the children such as sadness (27\%), anger (32\%), and being more withdrawn since the death (43\%) than the bereaved children reported about themselves (sadness: 8\%; anger: 1 child; being more withdrawn since the death: $5 \%$ )

Half of the participants reported changes in the children's relationships with peers. Some developed new relationships and stronger bonds with their current friends; others ended relationships and felt more distant from friends (Foster et al., 2012). Loneliness is a common feeling for children after the death of a sibling and it is related more to being alone with the experience than being physically alone (Nolbris \& Hellstrom, 2005). Children (33\%) reported changes in peer relationships more often than fathers (13\%) and mothers (14\%) (Foster et al., 2012). Parents may not have noticed the effects in the social relationships of the children in relation to changes in children's 
personalities and emotional wellbeing after the death of the sibling. Peer groups provide emotional refuge for bereaved children, especially for girls, who are more likely to express their feelings and elicit sympathy from others (Nolbris \& Hellstrom, 2005). Foster et al.'s (2012) study sample was primarily White, English speaking, and consisted of families whose sibling died of cancer. Also, this study was cross-sectional and the reports of changes in children may vary over time, hence, a longitudinal study would be beneficial.

A study by Youngblut and Brooten (2013) described the parents' reports of child responses to the death of a sibling in the NICU/PICU through qualitative interviews 7 months after the death. Half of the 24 deceased siblings were infants. Forty-six percent of the infants died secondary to congenital or chromosomal abnormalities. Twenty-seven parents reported on responses of 44 of their children. The children consisted of 10 preschool, 19 school-age, and 15 adolescents. Most of the parents were high school graduates (93\%), Catholic or Protestant/Christian (77\%), employed (85\%), married or partnered (63\%), and lower income (62\%).

The parents' comments were grouped into 6 themes: changed behaviors (37.5\%), not understanding what was going on (23.4\%), maintaining a connectedness with the sibling (14\%), not enough time to be with sibling and/or say goodbye (9.4\%), believing sibling is in a good place (9.4\%), and not believing sibling would die (6.3\%). Changed behaviors were mainly reported by parents of school-age children and adolescents, and not understanding what was going on was primarily reported by parents of preschoolers. Changed behaviors included children being distant from parents, not talking, not participating in activities, and avoiding things shared with their sibling (Youngblut \& 
Brooten, 2013). White parents commented the least (1.3/child) compared to Black and Hispanic parents (1.5/child for both). Half of the White parents commented about changed behaviors, with none about not understanding or sibling being in a good place. Black parents commented the most about lack of understanding and changed behaviors. All of the comments about the sibling being in a good place were made by Black parents, which might be due to their religious beliefs. Hispanic parents commented about changed behaviors and lack of understanding.

Parents whose child died in the PICU commented more on their surviving children than parents whose child died in the NICU. This could be due to the fact that the children spent more time with the siblings that were admitted in PICU versus NICU before their death. A limitation of this study (Youngblut \& Brooten, 2013) is that the data were obtained only from parent reports. Parents' perceptions of their children's responses have been found to differ, especially from older school-age and adolescent children's reports of their thoughts and feelings (Guite, Lobato, Kao, \& Plante, 2004). It is necessary to also obtain data directly from the children to have a clear insight on what their thoughts and feelings are after the death of their sibling and compare their data to the parent data. Parents may be so overwhelmed with their own grief that they might overlook their children's grief reactions.

Physical. Reactions to the death of a sibling vary and they include guilt, anxiety, loneliness, withdrawal, headaches, stomach aches, and pains (Sirkia, Saarinen-Pihkala, \& Hovi, 2000). Sirkia et al. (2000) conducted a retrospective analysis of the parents of 60 children with cancer (21 leukemia, 18 solid tumors, 21 brain tumors) who died while in terminal care, and of parents of 26 children who died of therapy-related complications 
(leukemia: 11, solid tumor: 10, brain tumor: 2, Fanconi syndrome: 1, aplastic anemia: 1, X-linked lymphoproliferative syndrome: 1) during active anticancer therapy.

Structured interviews with the mothers and fathers in the terminal care (37 parents) and in the active therapy (19 parents) groups were performed separately (Sirkia et al., 2000). Coping of the parents was evaluated by analyzing the occurrence of changes in the relationship between parents, physical and mental problems, return to work, selfreported recovery time, and outlook of the children. Differences between the parents in the terminal care group and those in the active therapy group were minimal with regards to physical and mental problems, self-recovery time, and return to work.

In the terminal care group of deceased siblings aged 0.2 to 27 years at death, parents reported problems in 14 (18\%) children which included fears (3), school related problems (3), depression (3), behavioral problems (2), problems with friends (1), pains (1), and bulimia (1) (Sirkia et al., 2000). In the active therapy group parents reported problems in 11 (32\%) of the 34 children which included depression (1), behavioral problems (4), problems with friends (1), pains (1), hypertension (1), and stuttering (1). The children in the active therapy group (32\%) tended to have more problems than those in the terminal care group (18\%). It is possible that many of the children's problems remained unrecognized by the parents due to their own emotional distress. The cause of death of the siblings was cancer. Death from unexpected causes (accident suicide, homicide) was not examined. Also, only parent reports of children's problems were obtained.

Psychological. Paris et al. (2009) studied self-reported grief and trauma among 26 children from 22 families, ages 9 to 18 years, whose sibling died. Seventeen of the 
siblings died from sudden death (accident: 8 , homicide: 4 , suicide: 1 , sudden illness: 4 ) and 5 from anticipated death (cancer: 2, heart disease: 2, chronic illness: 1 ). The majority of the deceased siblings were boys (17). Grief symptoms were measured with the child and adolescent forms of the Hogan Inventory of Bereavement (HIB) (Hogan, 1988; Hogan \& DeSantis, 1996). PTSD (Post-Traumatic Stress Disorder) symptoms were examined using the Impact of Events-8 (IES-8) (Dyregov \& Yule, 1995). Preliminary analysis revealed that scores on the HIB and the total stress score on the IES-8 were highly correlated. Hence, grief and PTSD were difficult to distinguish in this group. Differences in the mean grief and PTSD scores for anticipated versus sudden death could not be established since the sample is disproportionate with majority of the siblings dying from sudden death (Paris et al., 2009). An interesting finding is that boys and girls scored equally in terms of PTSD, but girls reported greater grief after the loss than boys. Adolescent girls may be more susceptible to emotional difficulties when a sibling dies (Sandler et al., 2010).

The researchers posited that girls may have responded more openly to the emotional and psychological questions of the HIB than boys, while both boys and girls responded similarly to the IES-8, which focuses on physiological and cognitive reactions (Paris et al., 2009). Researchers did not measure behavioral responses, and possibly included emotional reactions more characteristic of girls or that girls will admit to. The picture of boys' experiences may be incomplete. Further investigation is needed to determine if girls have more severe grief reactions or whether findings have to do with gender-socialized responding. A limitation in this study is the small sample size. 
Fanos et al. (2009) studied the long-term developmental consequences for surviving siblings of infants in the neonatal intensive care unit (NICU). The sample consisted of fourteen children (13 adults and 1 adolescent at interview) whose sibling had died in the NICU 9 to 20 years earlier. The participants ranged in age from 16 to 27 years (median age was 22 years). The deceased infants had lived from a few minutes to 30 days. Six participants were born after the death of their sibling. Eight participants were between 3 and 7 years old (median, 5 years) when their sibling died. One important finding is that the participants' recalled having a desire to become involved with their sibling.

Being able to hold and/or view the infant was highly valued by the survivor children. The effects of a sibling's death can still be experienced years later (Horsley \& Patterson, 2006). The participants stated that they found it difficult to comprehend the illness and death of their sibling, and needed help and support at different times throughout their development (Fanos et al., 2009). Grief in children is often cyclical, and as they move through the stages of development, they will revisit their previous feelings, thoughts, and memories associated with the death (Willis, 2002). Limitations in this study (Fanos et al., 2009) are the long time for recall, small sample size, and the participant's emotional, social, cognitive, and psychological development as well as other experiences they have endured since the death of the sibling (losses of other loved ones).

Sandler, Robinson, and Carter (2012) studied whether children were effectively processing the loss of their sibling in the NICU. Out of the 20 eligible families, only 1 family participated in the study. The study consisted of a single interview of a 10-yearold girl, Sarah, whose sister had died 20 months ago in the NICU. Sarah had physical 
contact with her sister before her death. Sarah had held her sister and fed her through a feeding tube when she was in the NICU. When her sister died, Sarah said she was "shocked, mad, and sad." The surviving children whose twin died in the perinatal period may be affected both by the loss of their companion and the grief of their parents (Bryan, 2003). She also stated that the only positive memory she had of her sister was that she was able to hold her (Sandler et al., 2012). As adults, surviving children treasure the memories of being involved with the sibling (Fanos et al, 2009).

Sarah also reported that she felt anxious about her parents dying and was unsure about whether she would want a new baby brother or sister (Sandler et al., 2012). The death of a parent or sibling during childhood can create worry about the vulnerability of other family members (Dowdney, 2008). Sarah wrote a song with her father to honor her sister's memory (Sandler et al., 2012). Maintaining an ongoing connection with the deceased through specific actions is an important part of successful adjustment after the loss (Field \& Friedrichs, 2004).

A study by Lohan and Murphy (2001) examined parents' perceptions of adolescents' grief responses at 4, 7, 12, and 24 months after the death of their sibling. Parents (34 fathers and 73 mothers) who ranged in age from 32 to 61 years were recruited through an examination of public death record data. The age of the deceased child ranged from 12 to 28 years, parental education ranged from 9 to 20 years, and time since the child's death to entry into the study ranged from 6 weeks to 7 months.

Only 107 of the 142 parents were chosen to be part of the study (Lohan \& Murphy, 2001) based on their responses to a question on the Parent Bereavement Survey (Murphy, 1991) which asked them to describe the difficulties that each of their living 
children had experienced since the death of their sibling. The researchers did not specify what was needed in the parent responses to be selected for this secondary analysis. Parents responded to this same question at all of the four time points. Sixty-percent of the children's deaths were caused by accidents, $14 \%$ by suicide, and $11 \%$ were homicides. Parents' descriptions of their adolescents' grief responses were grouped by cause of death and divided into groups by the gender of the parent.

The data revealed that most of the parents' concerns about their children did not change over time and that parents' descriptions of their surviving children's responses did not vary by the deceased sibling's cause of death (Lohan \& Murphy, 2001). In this study, mothers provided more descriptions of their children's grief reactions than fathers. The 27 mothers described their older sons' (age 15-19, n=28) behaviors as more negative compared to the fathers. Fathers reported spirituality issues and mothers did not. The mothers reported physical problems and self-destructive behaviors (alcohol and other substance abuse) in their older sons and the fathers did not. Mothers ( $n=14)$ of sons aged 10-14 years $(\mathrm{n}=15)$ reported fewer difficulties for this age group than her other children, which were similar to the father's descriptions. The mothers described an extensive array of behavioral responses (sad and depressed affect) for 10-14 year old surviving children compared to fathers. The data from mothers $(\mathrm{n}=29)$ describing their older daughters (age $15-19, \mathrm{n}=30$ ) were the most extensive and characterized by negative observations ("trouble being fully present in life"). Younger daughters (age 10-14) were described by mothers in the same way as older daughters. Daughters between the ages of 10 and 14 were described as having difficulties with their mothers, such as being resentful for the lack of attention their mothers gave them. 
Fathers $(n=5)$ did not mention sadness, depression, or unhappiness in their sons (age 10-14, n=6), but they did mention anger and fear (Lohan \& Murphy, 2001). Fathers $(n=9)$ mentioned affective components of depression (crying and sadness) for daughters between the ages of 10 and $14(n=10)$ contrary to what fathers listed as affective issues for younger sons (anger and fear). Fathers' $(n=16)$ descriptions of their daughters between the ages of 15 and $19(n=18)$ were those of adolescent girls dealing with serious issues (boyfriend problems that included abuse and violence). Even though this study is longitudinal, grief reactions in adolescents experiencing the violent death of a sibling should be examined for a longer period of time to determine if there is a change in the grieving pattern. Studies of adolescents experiencing the death of a sibling from other causes such as illness are needed. There is also a need for these data to be reported by the adolescents themselves.

After completing an evaluation of 26 studies, Foster and Gilmer (2008) concluded that the prevalence of continuing bonds is high among bereaved individuals. Research has focused on the importance of continuing bonds and how maintaining such bonds is essential to adaptation to loss (Asai, Fujimori, Akizuki, Inagaki, Matsui, \& Uchitomi, 2010; Packman et al., 2006). Continuing bonds are common in bereaved spouses (Field \& Friedrichs, 2004; Field, Gal-Oz, \& Bonanno, 2003), siblings (Packman et al., 2006), parents (Barrera et al., 2009; Doran \& Hansen, 2006; Ronen et al., 2009) and pet owners (Field, Orsini, \& Gavish, 2009).

In a classic study done by Hogan and DeSantis (1992), a convenience sample of 157 adolescents was obtained through an international support group of bereaved parents and children of deceased siblings. The participants were between 13 and 18 years at the 
time of the study, had a sibling die within the last 5 years prior to the study, and were not receiving professional mental health counseling. The majority of the sample was female (65\%), Protestant $(\mathrm{n}=72)$, and Anglo (96\%). The majority of the deceased siblings were male $(68 \%)$, died secondary to an accident (59\%), and were older than the surviving child $(60 \%)$.

Findings from this study (Hogan \& DeSantis, 1992) indicate that adolescent sibling bereavement is a process that is not restricted by time and bereaved children experience a persistent, ongoing attachment to their deceased siblings. Continuing bonds were defined by the researchers as an ongoing attachment with the deceased, categorized as: regretting (wishing to have a better relationship; wishing to continue the sibling relationship), endeavoring to understand (searching for reasons for the sibling's death), catching up (asking what heaven is like and bringing the siblings up-to-date on the surviving child's life), reaffirming (loving and missing the sibling), influencing (seeking direction from the sibling), and reuniting (waiting for a reunion in heaven). Limitations of this study are that the majority of the surviving children were female, White, and Protestant. Also, the majority of the deceased siblings were male and they died secondary to an accident. The sample was obtained through a mutual support group for bereaved parents; therefore, generalizations of the findings may be limited. Since this study (Hogan \& DeSantis, 1992) is cross-sectional, longitudinal studies are needed to determine if the ongoing attachment with the deceased siblings is persistent over time.

In a study by Forward and Garlie (2003), bereaved adolescents found comfort in knowing that their siblings were no longer suffering and were in a better place and that they would be reunited again. Children believed that there was an afterlife and they 
would be reunited with their brother or sister when they died. Imagining an afterlife provides the bereaved children with a sense of peace and wellbeing since the dead can have emotions and they can continue to enjoy their favorite activities (Dowdney, 2008).

Some children feel they are still connected to the deceased (Packman et al., 2006). It is now recognized that maintaining an ongoing connection with the deceased is integral to successful adjustment (Field \& Friedrichs, 2004). Continuing bonds can facilitate coping for children, comforting them after the death of the sibling.

A study done by Foster et al. (2011) compared how parents and children experienced continuing bonds in 40 families who lost a child to cancer. The researchers did not specify the number of deceased siblings. The sample in this study included 36 mothers, 24 fathers, 39 children. The majority of the mothers (78\%) and fathers (83\%) were Caucasian. The bereaved children $(n=40)$ were 8 to 17 years old. Data were gathered through the use of open-ended questions 6 to 19 months post-death of the sibling. The researchers found three major themes: (a) bereaved parents and children intentionally chose reminders of deceased children, (b) bereaved parents and children encountered non-purposeful reminders of deceased children, (c) family members experienced both comforting and non-comforting effects of these reminders. Ninetyseven percent $(n=96)$ of all families reported choosing at least one purposeful reminder of the deceased child. The percentages reported by the researchers do not correlate with the number of participants in the sample. Purposeful reminders (purposeful bonds) included photographs, videos, and communicating with deceased siblings through talking, writing letters, or praying. The researchers do not specify when and how often the participants 
chose the purposeful reminders. Some family members kept the deceased sibling's personal belongings such as clothing and toys.

Only $14 \%(n=14)$ of family members encountered involuntary or non-conscious reminders of their deceased sibling that occurred all of a sudden (Foster et al., 2011). Nine percent $(n=9)$ of the participants felt the deceased sibling's presence. One child said "I can smell her. She smells like clean-ness". The child reported that having this connection with the sibling made him or her feel better. The majority of the bereaved family members maintained continuing bonds with the deceased siblings. Fifty-seven percent experienced comforting effects from continuing bonds and $10 \%$ experienced discomforting effects, especially, when reminders brought to surface feelings of sadness (Neimeyer, Baldwin, \& Gillies, 2006).

More mothers than children reported communicating with the deceased, thinking about the deceased, and partaking in activities that the deceased liked more than the children (Foster et al., 2011). Children reported fewer purposeful bonds with the deceased sibling than parents. Children tended to remain connected in a more concrete manner (keeping deceased sibling's belongings). When the sibling became sick, and after their death, the parents were not able to tend to the child's emotional needs. Children endured the deterioration and death of the sibling and they witnessed their parents' suffering and grief, which explains why children reported fewer purposeful bonds with the deceased sibling than parents. Purposeful bonds could have elicited feelings of sadness and discomfort in the children. The researchers did not separate the themes that emerged during each interview according to the time when each respective interview was conducted (6 to 19 months after sibling death). Taking into account the 
pattern of themes that emerged over time can provide information regarding the pattern of grief for children.

Conceptual definition of grief disorder. Siblings normally spend the majority of their time together and their relationship often lasts a lifetime (Foster et al., 2011). In a study conducted by Lohan and Murphy (2001), families seem to have no resolution after the loss of a sibling, and a surviving child mentioned that he "lost his best friend." Children can be deeply affected after the loss of a sibling, to such an extent, that they might experience pathological or traumatic grief. Researchers separate normal grief from pathological or traumatic grief as different categories of experience enabling a diagnosis of a psychological disorder (Jacobs, Mazure, \& Prigerson, 2000).

\section{Prolonged grief/complicated grief disorder, post-traumatic stress disorder}

and depression in bereaved children. A study by Spuij et al. (2012) found that symptoms of prolonged grief disorder (PGD) were different from those of bereavementrelated depression and PTSD in bereaved children and adolescents. Symptoms of PGD include trouble accepting the death, persistent and disruptive yearning, detachment, and a feeling that the loved one's death has severely affected one's perception of self, life, present, and future to the point of impairment beyond 6 months after the loss (Shear et al., 2011). The DSM-4 (APA, 2000) describes symptoms of posttraumatic stress disorder (PTSD) subsequent to experiencing an event involving actual or threatened death that include avoidance, intrusive thoughts, and increased arousal.

The sample in this study (Spuij et al., 2012) consisted of 197 children between the ages of 8 and 12 years and 135 adolescents between the ages of 13 to 18 years affected by the death of a parent, sibling or close relative with a mean time since loss of 
31 months for the children and 34 months for adolescents. The majority of the children $(161,82 \%)$ and adolescents $(105,78 \%)$ had experienced the death of a parent, most often the father (123 children, 63 adolescents). Almost half of the participants had been recruited by lay mental health care workers who organized support groups for bereaved children and adolescents. Other participants were recruited via outpatient mental health care clinics in the Netherlands. Most had suffered the death of a parent due to an illness.

Research on PGD has mainly been done in adult samples making this one of the few studies with children (Spuij, 2012). Findings show that children's PGD, depression, and PTSD factor scores were significant predictors of their functional impairment. In the adolescent sample, the PTSD factor was the only factor explaining unique variance in functional impairment. A limitation in this study is that the sample consisted of a heterogeneous group of children, with the majority of children suffering the death of a parent, instead of focusing on children bereft of the same relative, for example, parents or siblings. Also, average of 3 years post death.

Melhem, Mortiz, Walker, Shear, and Brent (2007) described the phenomenon of complicated grief (CG) and its correlates in parentally bereaved children and adolescents. A sample of 129 children, 7 to 18 years old, were recruited from 89 families in which a parent died by suicide $(n=33,25.6 \%)$, accident $(n=31,24 \%)$, or unexpected natural death $(n=65,50.4 \%)$. Most of the deceased parents were male, White, and middle-class. The mean time since the death of the parent was 8 months.

CG is described as a group of symptoms that include longing and searching for the deceased, purposelessness and futility about the future, difficulty accepting the death, feeling insecure and not in control, and angry and bitter over the death (Prigerson, 
Bridge, Maciejewski et al., 1999). CG differs from depression and anxiety (Boelen \& Van Den Bout, 2005; Latham \& Prigerson, 2004).

Results of the study (Melhem et al., 2007) indicated that when controlling for depression, anxiety, and PTSD, CG scores remained significantly correlated with functional impairment which suggests that $\mathrm{CG}$ is different from the other disorders. A study by Dillen, Fontaine, and Verhofstadt-Deneve (2009) support the distinctiveness of CG from depression and anxiety in adolescents experiencing the loss of a grandparent. Higher CG scores were associated with suicidal ideation (Melhem et al. 2007).

Surprisingly CG scores were not higher in children who lost a parent to accident versus sudden natural death or suicide. CG scores in children were associated with increased depressive, anxiety, and PTSD symptoms, and suicidal ideation. In adults, CG peaked at 6 months after the death and remained high 25 months and after (Prigerson et al., 1997). A longitudinal study would allow the researches to examine the pattern of CG in children and adolescents over time. Also, the children and adolescents in the sample only experienced a sudden parental death. The impact of other types of losses, such as those from chronic illness or sibling death, was not examined.

\section{Summary}

Children's understanding of spirituality is parallel with their cognitive development and is developmentally sequenced. The development of children's faith occurs in stages consistent with Erikson's theory of psychosocial development, Piaget's theory of cognitive development, and Kohlberg's theory of moral development. These theories portray how children's thinking evolves and becomes more abstract allowing them to increase their comprehension of religious concepts (Barnes et al., 2000). 
Children create their own religious traditions based on their parents' religions and their individual needs. Barnes, Plotnikoff, Fox, and Pendleton (2000) state that religious traditions can "provide structures for moral development, and for the socialization of the child into different ideals of personhood and behavior" (p. 900). Children develop a set of traditions that consists of elements learned at home as well as practices and images that they learn from the media and friends (Pike, 2009). Children's religious practices and beliefs have been shaped and reinterpreted in their daily interactions with adults and peers and through the internet and television (Ridgely, 2012).

Woolley and Phelps (2000) found that children as young as 4 to 6 years view both the mind and an agent (God) as necessary components of the prayer process and that praying could be done anywhere. Children between the ages of 4 and 8 believe in the efficacy of prayer (Wolley \& Phelps, 2000), and they show a significant decrease in beliefs about the efficacy of wishing as they become older (Woolley, Phelps, Davis, \& Mandell, 1999). These findings suggest that children may have a more intricate concept of prayer than previously assumed.

Nowadays children are less aware of the dying process. In the past, individuals died at home surrounded by friends and family. Currently, many people die in hospices, hospitals, and nursing homes, and children may not be a part of the dying process. They may view death as a rare event. Death of a child or parent during childhood is less common today.

There are four mechanisms associated with children's understanding of death: irreversibility, finality, inevitability, and causality (Willis, 2002). Children's understanding of these four components is directly related to their developmental level 
when the death occurred. A study conducted by Bonoti, Leondari, and Mastora (2013) found that children's understanding of death is associated with age and past experience. Children who had a personal death experience had a better understanding of death compared to children with no prior death experience.

Religious experience may affect children's understanding of death (Bering et al., 2005). A study by Sagara-Rosemeyer and Davies (2007) found a strong connection between the children's religion and their notion of death. Two studies by Harris (2011), one conducted in Spain and the other in Madagascar, found that as children progress through the stages of development their biological notion of death is enhanced with the religious conception that there is life after death.

Many of the studies mentioned describe how children and adolescents rely on their faith to navigate through difficult circumstances such as physical and mental illness (Josephson \& Dell, 2004; Koenig, 2003; Mueller et al., 2001) and the death of a loved one. Various researchers agree on the protective role that spirituality and religious coping have in adolescents' lives (Grossoehme, 2007; Grossoehme, 2008; Talik, 2011).

Kamper, Cleve, and Savedra (2010) found that the majority of the children with advanced cancer relied on God or a Divine power to help them feel better and shorten their hospital stay. When facing chronic and severe stress, using religion as a positive coping mechanism helps the individual perceive the situation in positive manner which promotes psychological health (Folkmam \& Greer, 2000).

On the contrary, in an exploratory study by Batten and Oltjenbruns (1999) some of the adolescents expressed anger after the death of their brother or sister while others had increased belief in the higher power. Cotton et al. (2005) examined the effects of 
spirituality and religion on depressive symptoms and health risk behaviors with White adolescents. Some of the adolescents who felt that religion was important to them had more depressive symptoms. The samples in these studies were predominantly White and the majority of the children were of Christian faith. It is important to identify religious beliefs and practices that are detrimental and/or helpful to children after the death of a brother or sister, parent, or grandparent while taking into account the child's age, gender, race/ethnicity, and religion.

In the past, the deaths of children were not an unusual happening. In underdeveloped countries were death rates are high the death of a child is seen as a natural occurrence with mourning lasting only a few days (Parkes et al., 1997). In developed and developing countries changes in nutrition, sanitation, and medical advances have greatly decreased the mortality rate in children. Childhood deaths are often seen as unnatural and tragic. The death of a child is given unique significance across cultures (Parkes et al., 1997). The child's gender, age, and cause of death have a direct effect on the meaning given to such a loss and determine the norms for appropriate grieving behaviors within a particular culture. After reviewing the literature little information was found regarding death rituals performed for deceased children.

After the death of a loved one a death ritual can legitimize grief, provide an arena where the death is acknowledged and its finality is accepted, and creates a safe place for the mourner to express emotion. Weeks (2004) explains that, after the individual dies and before the funeral or memorial services, those with common bonds, such as religion or ethnic origin, perform rituals to recognize the death and pay tribute to the deceased. There are very few studies that examine the impact that death rituals have on children 
after the death of their sibling. The participation of children from different cultures and religions in death rituals and its effects should be examined, specifically after the death of a sibling.

Research that focuses on bereaved children and adults finds those deaths which were unexpected, in the hospital versus the home, more recent (within $1-1.5$ years) and associated with prolonged illness and/or suffering may be associated with more grief reactions (Barry et al., 2004). Current research on sibling bereavement has found an array of grieving responses: emotional (depression, sadness, anxiety, feelings of guilt), behavioral (sleep disturbances, acting out), social (withdrawal, loneliness), and deterioration in school performance (Fanos et al., 2009). Bereaved children may not find the emotional support they need from their parents since they are grieving and engulfed in their own pain. Also, some children may not disclose their feelings or seek support because they think that they have to protect their grieving parents (Dyregrov \& Dyregrov, 2005).

The death of a parent has been labeled as one of the most stressful events that a child can experience (Harrison \& Harrington, 2001). A number of prospective and retrospective studies have proven that children that have experienced the loss of a parent are at risk for psychological problems which consist of depression, posttraumatic stress disorder, substance abuse, and health risk behaviors (Brent, Melhelm, Donohoe, \& Walker, 2009; Maier \& Lachman, 2000). Children that have experienced the death of parent as a child report negative ramifications such as poorer health outcomes and diminished educational attainment in their adult lives (Mack, 2001; Maier \& Lachman, 2000; Marks, Jun, \& Song, 2007). 
The death of a grandparent is one of the most frequent types of losses among adolescents (Westerink \& Stroebe, 2012). The degree of anxiety and grief that an adolescent experiences after the death of a grandparent is directly related to the quality of their relationship before the death. There is a paucity of research on the consequences that the death of a grandparent has on younger children.

\section{Issues and Gaps}

Lack of a clear and consistent definition of spirituality hampers research in this area. Some describe spirituality as being different from religion because it is not based on a discrete value system (Farrell et al., 2008). In contrast, others argue that even though spirituality and religiosity have distinct characteristics, they are highly intertwined (Good et al., 2011; Walker et al., 2009).

In studies of children's religious beliefs and practices, children's beliefs were not directly measured relying solely on parent reports of their child's current beliefs. Children's reports of their beliefs would provide a clearer understanding of the association between their beliefs and their outcomes. Bamford and Lagattuta 2010 found that younger children with high exposure to religious training had more sophisticated prayer and God concepts, suggesting that religious and spiritual experiences may enhance children's development of these concepts.

Researchers' thoughts vary regarding the age at which children attain a clear notion of death. Contrary to Nagy's views (1948) that toddlers and preschoolers have no notion of death, findings of a study by Slaughter and Griffiths (2007) suggest that fundamental ideas of the irreversibility of death begin to form as early as age 5 or 6 . Bonoti, Leondari, and Mastora (2013) found that children's understanding of death is 
associated with age and past experience. Children who had a personal death experience had a more accurate understanding of death than children with no prior death experience. Very little research on specific death rituals performed for deceased children has been published.

Most of the studies pertaining to child and adolescent bereavement have concentrated on the death of a parent, not a grandparent or sibling (Sood, Razdan, Weller, \& Weller, 2006). Ens and Bond (2005) studied adolescent's death anxiety and bereavement but did not consider the quality of the grandparent-adolescent relationship. There is limited research on the spiritual coping strategies used by children after the death of a parent, grandparent, or sibling.

The majority of the research done in children's bereavement focuses on siblings and parents who have died of cancer excluding other causes of death. Findings may differ for children whose parent, grandparent, or sibling died from other chronic illnesses, or other causes (e.g. trauma or suicide). Most study participants were Protestant and Caucasian. Findings from these studies may not apply to families who practice other religions and who are from different cultures.

Methodological differences in time of assessments, age of participants, and instruments used make it difficult to pinpoint specific grieving patterns in children (Barrera et al., 2013). Also, most of the studies vary with respect to elapsed time after the death, which may have an effect on the grief, personal growth, anxiety, and depression that the child experiences.

The current literature does not emphasize the spiritual coping strategies that a child uses after the death of a parent or sibling. There is limited research that describes 
the effects that the death of a grandparent has on the child and there are no studies with respect to spiritual coping strategies used by the child after such a loss. There is also scant research regarding the effects of children's participation in religious rituals and practices after the death of a parent or sibling, and none after the death of a grandparent.

Most of the data have been obtained only from parent reports. In a study by Guite, Lobato, Kao, and Plante (2004), parents' perceptions of their children's responses differed from responses reported by older school-age children and adolescents. It is necessary to also obtain data directly from the children to have a clear insight on what their thoughts and feelings are after the death of their sibling and compare their data to the parent data. Parents may be so overwhelmed with their own grief that their children's grief reactions receive little attention.

Research that identifies differences in spiritual coping strategies used by children across age groups, gender, race/ethnicity, participation in religious rituals and practices while taking into account the relationship of the deceased to the child (parent, grandparent, or sibling) is lacking. The relationship between children's spiritual coping strategies, and grief, personal growth, depression, and anxiety, and depression after the death of a parent, grandparent, or sibling has not been examined. This study addressed these gaps. 


\section{CHAPTER III}

\section{DESIGN AND METHODS}

The CDC reported a total of 23,215 deaths in infants under 1year of age; annual death rates of 3,830 per 100,000 children 1 to 4 years, 5,250 per 100,000 children 5 to 14 years, and 9,586 per 100,000 adolescents 15 to 19 years; and total of 2,626,418 adult deaths. Despite these numbers, research on children's experiences of death of a parent, grandparent or sibling during childhood is limited. The purpose of this correlational study was to identify 1) differences in the spiritual coping strategies used by children across age groups, gender, race/ethnicity, participation in religious rituals and practices, and the relationship of the deceased to the child (parent, grandparent or sibling), and 2) the relationship between children's spiritual coping strategies and grief, personal growth, anxiety, and depression after the death with and without controls for child's age, gender, race/ethnicity, participation in religious rituals and practices, and relationship to the deceased.

\section{Setting}

Children and their parent(s) were recruited for this study through Nicklaus Children's Hospital, Baptist Congregational Health, Children's Bereavement Center, Gentiva Hospice Foundation, Season's Hospice, Tomorrow's Rainbow, the YoungblutBrooten sibling study sample, snowball sampling, and personal contacts. Nicklaus Children's Hospital is a licensed free-standing 289-bed specialty hospital exclusively for children up to 21 years old. Baptist Congregational Health provided health education and supportive care of the needy in Miami-Dade County via bereavement support, care teams and faith-based health support groups programs until 2015 when it closed. 
The Children's Bereavement Center provides free peer support groups at 3 different locations for children, young adults, and adult caregivers separately after the death of a loved one. The groups are open to anyone who has lost a child, parent, grandparent, sibling, relative, or friend as a result of illness, accident, suicide, or homicide. Losses can be recent or in the years past. Peer support groups meet in the evening each week throughout the school year and are non-denominational. All groups and activities are facilitated by trained volunteers and the professional staff of the Children's Bereavement Center.

Under the direction of a physician, Gentiva Hospice's team of clinicians offers a caring atmosphere that incorporates the social, medical, emotional, and spiritual aspects of treatment. The team consists of medical directors, expert nurses, social workers, hospice aides, spiritual care coordinators, and specially trained volunteers. Gentiva hospice provides clinical care with emphasis on pain and symptom management, emotional support for patients and family members, and a comprehensive bereavement program.

Tomorrow's Rainbow was created for children who experienced the death of a loved one. It incorporates miniature horse interactions with therapeutic play areas and facilitated peer support. Tomorrow's Rainbow strives to provide grieving children, teens, and their families an emotionally safe environment for hope and healing through guidance, education, and support.

The Youngblut-Brooten Sibling study focused on health and functioning of children age 6 to 18 , during the first 13 months after the death of a sibling in the NICU, PICU, and Emergency Room of hospitals throughout Florida. Also included were 
children whose technology dependent siblings were discharged to die at home. One child in families who had completed their 2, 4, 6, and 13 month data points were eligible for this study.

\section{Sample}

A convenience sample of 97 children between the ages of 8 and 18 years and their parents/legal guardians were recruited. One child from each family that met the inclusion criteria participated in the study. If qualifying families had more than one child who could participate, names of the eligible children were written on slips of paper and the name of the child participant was randomly chosen.

Inclusion criteria for the surviving child included having experienced the death of a parent, grandparent, brother, or sister within the past two years, able to read and write English, and currently in their age appropriate grade level in school +/- 1 year. This age group was chosen because they had the cognitive and verbal capacity that was necessary to complete the questionnaires. The child's parent/legal guardian had to be 18 years of age or older and able to read English and/or Spanish to participate. The PI is fluent in Spanish and English and was able to communicate with parents/guardians who were Spanish speakers.

Exclusion criteria for the surviving child included: 1) conditions that render a child unable to participate verbally - e.g., cerebral palsy, severe brain damage, severe autism; 2) living in foster care before or after the death; 3) history of psychiatric conditions; and 4) death of more than one family member in the previous 2 years because the child will be dealing with multiple deaths at the same time. 
Power Analysis was conducted for each research question, setting alpha at .05 and power at $80 \%$. For RQ 1, oneway ANOVAs with a maximum of 3 groups and a medium effect of .30 needed a sample of 111. For RQ 2, correlations of .30 required a sample of 84. For RQ 3, multiple linear regression with a medium effect of .15 and 8 independent variables required a sample of 109; with 9 independent variables, it requires 114 . A final sample of 120 was planned to provide enough power to conduct the planned analyses.

\section{Procedure}

Institutional Review Board (IRB) approval was obtained from Florida

International University (FIU), and Nicklaus Children's Hospital (NCH) through WIRB. A number of different sites were used for recruitment. The procedure for getting names and contact information varied by site.

Flyers explaining the nature of the study, inclusion and exclusion criteria, the PI's name and contact information were posted at local churches and Nicklaus Children's Hospital. The flyer was posted through FIU's daily email announcements. An e-mail containing the same information was to be sent by Peter Willig, Chief Operating Officer of the Children's Bereavement Center to participating families.

The PI distributed flyers in support groups headed and overseen by Chaplain Shirley Brown (Senior Congregational Health Chaplain) from Baptist Congregational Health, and special events for bereaved families offered by the Children's Bereavement Center, Baptist Congregational Health, and Tomorrow's Rainbow. Families who were interested in participating in the study were asked to contact the PI with the information on the flyer. Families recruited via snowball sampling and Genteva Hospice received flyers at the point of contact. 
At Nicklaus Children's Hospital, the PI received a list of names of deceased child patients from the social work department. The PI identified families of children (newborn to 18 years of age) on the list who had died at $\mathrm{NCH}$ in the previous 6 months to 2 years who had at least 1 surviving child between the ages of 8 and 18 years. The PI contacted the families by phone and screened for inclusion and exclusion criteria, explained the study, answered any questions they had, and determined whether the family was willing to participate. If the family was willing to participate, the PI scheduled the interview.

Personnel who collected data on the Youngblut-Brooten sibling study (Donna Charles, Lynn Seagrave, and Rosa Roche) identified families who had completed data collection for the Youngblut-Brooten study. Rosa Roche contacted the family and asked for their permission to provide the family's name and contact information to the PI. Agreement to be identified to the PI did not indicate agreement to be in this study. The PI or research assistant (RA) contacted the family by phone, screened for inclusion and exclusion criteria, explained the study, answered any questions they had, and determined whether the family was willing to participate.

The PI scheduled an appointment with the parent(s)/legal guardian(s) that had agreed to participate in the study to go to the family's home or another quiet and private place where they felt comfortable. In the home, the PI and/or RA introduced themselves, explained the study, and answered any questions the child had. The consent form was read to the parents in English or Spanish, depending on their preferred language, and they were asked to sign the consent form for their child and themselves to participate in the study. After the parent signed the consent, the child was asked about his/her willingness to participate in the study. If the child agreed to participate, the assent form was read to 
them and they were asked to sign the assent/consent form. If the parent/legal guardian did not give consent, their child was not asked to participate. Children who were 18 years old were asked to sign the parent consent form for their own participation.

Parents/legal guardians were informed that the children's answers were confidential and that they would not have access to these data. The children were told that they did not have to talk to their parents about their study responses so they felt comfortable answering the questionnaires. If concerned for the child's physical and/or mental health, the PI and/or RA spoke to the parents and recommended that the child be evaluated by their primary care provider.

Parents completed a demographic information form while their child completed their questionnaires in a separate part of the house (not within hearing distance of the parent(s) and other children). The PI and/or the RA read the items to the child to reduce the time for the interview and the effect of the child's reading ability on their responses. Each child was assigned a unique study identification (ID) number that was placed on every page of the forms. The child's ID number and name was stored separately from the participant $\log$ where the parent(s) and child's names, phone number, and address were recorded. The participant log, the data and the signed consent and assent forms were kept in separate locked drawers in the PI's office. The electronic file of data was kept in the PI's computer which is password protected and a back-up copy was stored in the locked drawer with the data. All study-generated materials were accessible only to research personnel and the PI's dissertation committee chair. 


\section{Measures}

\section{Demographic information}

The parents/legal guardians completed a demographic form that included their age, race/ethnicity, education, religion, marital or partner status, annual income, gender and age of each surviving child in the family; date of data collection; the deceased's age, sex, cause, date, and place of death, relationship to child; and whether the child had lived with the deceased. On this demographic form, parents were asked to identify religious rituals and practices observed for the deceased, when these activities occurred, and in which activities the study child participated.

\section{Person (child) factors}

The child's age and the child's gender were obtained from the parent's demographic form. The child's race/ethnicity was measured with 2 questions: 1) "Are you Hispanic?" and 2) "Which of the following best describes your race: White, Black (African American or African Caribbean), Asian, Native American Indian, or Alaskan Native?" From the responses to these two questions, race/ethnicity categories were created: Hispanic, White non-Hispanic, Black non-Hispanic, Asian non-Hispanic, and Native Alaskan or American Indian non-Hispanic. A set of dummy coded variables for use in the multiple regression analyses was created based on the racial distribution in the sample. White non-Hispanic was the suppressed group.

Participation in religious rituals and practices. Children were asked if they participated in any religious rituals and practices for the deceased and if so, to specify the religious rituals and practices in which they participated. The child's participation in any such rituals and practices was coded as 0 "No" or 1 "Yes." 
Relationship of the deceased. It was coded as 1 "sibling," 2 "parent," and 3 "grandparent." Two dummy coded variables were created with "grandparent" as the suppressed group and used in the multiple regression analyses.

Spiritual coping strategies. They were measured with the Spiritual Coping Strategies Scale (SCSS) (Baldacchino \& Buhagiar, 2003). The 20-item instrument is composed of two subscales: Religious coping strategies (9 items) and Spiritual coping strategies (11 items). Participants rate each item on a 4 point scale ranging from 0 "never used" to 3 "used often" in the previous 2 weeks, with higher scores indicating greater use of spiritual and religious coping strategies. Baldacchino and Buhagiar (2003) reported Cronbach's alphas of .82 for the religious coping strategies subscale and .74 for the spiritual coping strategies subscale and test-retest reliabilities of $r=.47$ after a 3-week interval. Construct validity of the SCSS is supported by a correlation of .40 between the original SCSS and the Spiritual Well Being Instrument in adults (Baldacchino \& Buhagiar, 2003). The SCSS has not been used in children. The PI talked to children (known to the PI) between the ages of 8 to 18 about the SCSS items for relevance and understanding. These children verbalized and demonstrated appropriate understanding of the instrument's items. In the current study, internal consistency reliability was $.85, .74$, and .85 for the total SCSS, Spiritual Coping, and Religious Coping scales, respectively.

Children's grief and personal growth. The Hogan Inventory of Bereavement (HIB), a measure of children's grief and personal growth, has a child version for 8- to 12year-olds and an adolescent version for 13- to 18-year-olds that differ only in reading level. The instrument is composed of two subscales: Grief (alpha= $.90-.95,24$ items) and Personal Growth (alpha $=.88-.92,22$ items) (Neimeyer \& Hogan, 2001). Children rated 
each item on a 5 point scale ranging from 1 "Does not describe me at all" to 5 "Describes me very well" over the past 2 weeks; higher scores indicate greater grief and personal growth (Hogan \& De Santis, 1996). Correlations between adolescent death anxiety after a grandparent's death and their HIB grief $(r=.40)$ and personal growth $(r=.21)$ scores support the subscales' validity (Ens \& Bond, 2005). In the current study, Cronbach's alphas were .85 for the Personal Growth subscale and .90 for the Grief subscale.

Child mental health. Child mental health had 2 indicators: child anxiety and child depression. Anxiety was measured with the Spence Children's Anxiety Scale (SCAS) which is appropriate for children between 8 and 18 years old. The scale consists of 44 items in six domains: generalized anxiety, panic/agoraphobia, social phobia, separation anxiety, obsessive compulsive disorder, and physical injury fears. Children were asked to rate the degree to which they experienced each symptom in the past 2 weeks from 1 "never" to 4 "always." The SCAS takes about 10 minutes for children to complete. The internal consistency reliability of the total scale was high with a Cronbach alpha of .93. In a study by Spence, Barrett, and Turner (2003) of 875 adolescents aged 13 and 14 years, convergent validity of the SCAS was supported by its correlation of .75 with the Revised Children's Manifest Anxiety Scale (RCMAS) scores. Muris, Schmidt, and Merckelbach (2000), in a study of 1011 children (524 boys, 487 girls) from the Netherlands ranging in age from 7 to 19 years, found a strong correlation $(r=.89)$ between the Screen for Child Anxiety Related Emotional Disorders [SCARED] and the SCAS, supporting the concurrent validity of the SCAS. In the current sample, alpha for the total SCAS was .74. 
The Children's Depression Inventory (CDI) measures depression in children between 7 and 17 years old. The CDI consists of 27 items (symptoms) with a 0-2 response scale; each response number is accompanied by a phrase about that symptom. Children chose the number of the phrase that described their symptoms over the past 2 weeks. Overall total scores of depressive symptomatology range from 0 to 54, with higher scores indicating more severe depressive symptoms. The CDI can be completed in approximately 15 minutes.

In a study by Saylor, Finch, Spirito and Bennett (1984) of 294 "normal” school children and 269 children who were psychiatric inpatients, the CDI's internal consistency was .94 for the "normal" school children and .80 for children with psychiatric diagnoses. A one week test-retest correlation of .87 was found for the emotionally disturbed children. In a study of 875 Canadian children, aged 10 to 17 years, Friedman and Butler (1979) found that higher CDI scores were related to lower self-esteem scores on the Piers-Harris Children's Self Concept Scale, $r=.66$ (Kovacs, 1980), supporting the CDI's validity. In the current sample, the CDI's internal consistency reliability was .87 .

\section{Data Management}

The PI made sure that the pre-assembled packet contained the necessary forms by maintaining a checklist of the forms needed before going to the family's home. A master log book was kept which included the assigned child ID, date when the interview was completed, and by whom. The PI and/or RA placed missing codes where parents/legal guardians or children chose not to respond and assigned numbers for open ended items such as the religious rituals and practices that occurred for the deceased and in which children participated after the death of a parent, grandparent or sibling. 
Child and parent/legal guardian data were entered into one SPSS file labeled with the child's ID. The initial analyses of descriptive statistics and frequencies were used to investigate the distribution and range of each variable. Any errors in the data were corrected before analyses to address the research questions were conducted. Coefficient alphas were calculated for all multi-item scales and subscales. 


\section{CHAPTER IV}

\section{RESULTS}

The purpose of this correlational study was to identify 1) differences in the spiritual coping strategies used by children across age groups, gender, race/ethnicity, participation in religious rituals and practices, and relationship of the deceased to the child (parent, grandparent or sibling), and 2) the relationship between children's spiritual coping strategies with grief, personal growth, anxiety, and depression after the death with and without controls for child's age, gender, race/ethnicity, participation in religious rituals and practices, and relationship of the deceased to the child. This chapter presents the description of the sample and statistical results for each research question.

\section{Description of the sample}

The sample consists of 97 children who had experienced loss of a parent $(n=39)$, grandparent $(\mathrm{n}=31)$, or sibling $(\mathrm{n}=27)$ within the past 2 years. Children ranged in age from 8 to 18 years, with most being school age; about half were boys. Most were Hispanic, followed by Black, then White; 1 child was a Pacific Islander (Table 1). Most children participated in religious rituals around the time of their parent's, grandparent's, or sibling's death. Almost half of the families were Catholic. More than half of the children had suffered the death of their loved one within a period of 11 months or less. The majority of the children lost their loved one due to cancer versus other causes of death. More than half of the deceased died in the hospital. From the group of families that reported their income almost half of them had an income of $\$ 30,000$ or more with most of them between $\$ 40,000$ and $\$ 60,000$ per year. Relationship-to-the-deceased 
groups were not statistically different on age, gender, race/ethnicity, participation in rituals, and time between the death and data collection interview.

\section{Differences in spiritual coping by person factors}

Research Question 1: Are there differences in the spiritual coping strategies used by children across age groups, gender, race/ethnicity, participation in religious rituals and practices, and the relationship of the deceased to the child (parent, grandparent or sibling)?

Hypothesis 1. There will be differences in the use of spiritual coping strategies across children's age groups, gender, race/ethnicity, participation in religious rituals and practices, and the relationship of the deceased to the child (parent, grandparent or sibling).

Children's use of spiritual coping strategies (total) and use of spiritual and religious coping strategies was compared by groups for child age, gender, race/ethnicity, participation in religious rituals and practices (yes/no) and relationship of the deceased to the child with oneway ANOVA tests (Table 2). When the oneway ANOVAs of variables (race/ethnicity, relationship of the deceased) with 3 groups were statistically significant, Scheffe post-hoc tests were used to determine which groups were significantly different from the other(s). Significant differences on religious coping were found between children who did and did not participate in religious rituals around the time of the death. Children who participated in religious rituals used less religious coping strategies than children who did not participate. None of the other differences were statistically significant. 
Table 1

Sample Demographics

\begin{tabular}{|c|c|c|c|c|c|}
\hline Characteristic & $\begin{array}{c}\text { Total Sample } \\
\qquad(N=97)\end{array}$ & $\begin{array}{l}\text { Grandparent } \\
(n=31)\end{array}$ & $\begin{array}{l}\text { Parent } \\
(n=39)\end{array}$ & $\begin{array}{l}\text { Sibling } \\
(n=27)\end{array}$ & Statistic \\
\hline \multirow{3}{*}{ Age } & $M(S D)$ & $M(S D)$ & $M(S D)$ & $M(S D)$ & $F$ \\
\hline & $11.9(3.31)$ & $12.2(3.39)$ & $11.0(3.05)$ & $12.8(3.41)$ & 2.72 \\
\hline & $N(\%)$ & $\mathrm{n}(\%)$ & $\mathrm{n}(\%)$ & $\mathrm{n}(\%)$ & $\chi^{2}$ \\
\hline Age Groups & & & & & 7.17 \\
\hline School age & $59(60.8)$ & $16(51.6)$ & $30(76.9)$ & $13(48.1)$ & \\
\hline Adolescents & $38(39.2)$ & $15(48.4)$ & $9(23.1)$ & $14(51.9)$ & \\
\hline Gender & & & & & 1.12 \\
\hline Males & $51(52.6)$ & $18(58.1)$ & $21(53.8)$ & $12(44.4)$ & \\
\hline Females & $46(47.4)$ & $13(41.9)$ & $18(46.2)$ & $15(55.6)$ & \\
\hline Race/Ethnicity & & & & & 2.15 \\
\hline White & $13(13.4)$ & 4 (12.9) & $6(15.4)$ & $3(11.1)$ & \\
\hline Black & $22(22.7)$ & $9(29.0)$ & $9(23.1)$ & $4(14.8)$ & \\
\hline Hispanic & $62(63.9)$ & $18(58.1)$ & $24(61.5)$ & $20(74.1)$ & \\
\hline $\begin{array}{l}\text { Participation in } \\
\text { religious rituals }\end{array}$ & & & & & 1.08 \\
\hline Yes & $75(73.3)$ & $22(71.0)$ & $31(79.5)$ & $22(81.5)$ & \\
\hline No & $22(22.7)$ & $9(29.0)$ & $8(20.5)$ & $5(18.5)$ & \\
\hline
\end{tabular}


$* \mathrm{p}<.05 \quad * * \mathrm{p}<.01$

Table 2

Differences in Spiritual Coping by Person Factors

\begin{tabular}{|c|c|c|c|c|c|c|}
\hline & Spiritual & & Religious & & Total SCS & \\
\hline Characteristic & $\mathrm{M}$ [SD] & F value & M [SD] & F value & $\mathrm{M}$ [SD] & $\mathrm{F}$ value \\
\hline Age group & & 1.54 & & 1.84 & & 1.94 \\
\hline School Age & $25.2(4.84)$ & & $16.0(6.34)$ & & $41.1(9.04)$ & \\
\hline Adolescent & $23.5(5.98)$ & & $13.2(7.70)$ & & $36.6(12.16)$ & \\
\hline Gender & & 0.06 & & 0.81 & & 0.57 \\
\hline Males & $24.5(4.10)$ & & $14.3(7.43)$ & & $38.8(10.06)$ & \\
\hline Females & $24.5(6.49)$ & & $15.5(6.50)$ & & $40.0(11.08)$ & \\
\hline Race/Ethnicity & & 0.95 & & 2.93 & & 0.38 \\
\hline White & $25.3(4.87)$ & & $13.2(7.18)$ & & $40.0(10.04)$ & \\
\hline Black & $23.2(6.88)$ & & $17.9(6.62)$ & & $41.1(12.03)$ & \\
\hline Hispanic & $24.9(4.77)$ & & $14.1(6.91)$ & & $39.0(10.04)$ & \\
\hline $\begin{array}{l}\text { Participation in } \\
\text { religious rituals } \\
\text { and practices }\end{array}$ & & 0.08 & & $2.24 *$ & & 1.51 \\
\hline Yes & $24.4(5.52)$ & & $13.8(7.15)$ & & $38.2(10.99)$ & \\
\hline No & $24.6(4.79)$ & & $17.5(5.60)$ & & $42.1(8.16)$ & \\
\hline $\begin{array}{l}\text { Relationship of } \\
\text { deceased }\end{array}$ & & 1.25 & & 0.94 & & 1.33 \\
\hline Parent & $24.8(4.10)$ & & $14.5(7.29)$ & & $39.4(9.34)$ & \\
\hline
\end{tabular}


Grandparent $\quad 23.2(6.57)$

Sibling
$13.9(7.76)$

$16.3(5.33)$
$37.1(12.33)$

$41.6(9.72)$

$* \mathrm{p}<.05 \quad * * \mathrm{p}<.01$

\section{Spiritual coping strategies, grief, and outcomes}

Research Question 2: Are children's spiritual coping strategies related to their grief, personal growth, anxiety, and depression after the death of a parent, grandparent, or sibling? Is children's grief related to their personal growth, anxiety, and depression after the death of a parent, grandparent, or sibling?

Hypothesis 2a. Children's use of spiritual coping strategies will be related to child grief, personal growth, anxiety, and depression.

Pearson's product moment correlations were used to determine the strength and direction of the relationship between spiritual coping strategies and the outcomes of grief, personal growth, anxiety, and depression (Table 3). Greater use of spiritual coping strategies overall and the specific spiritual coping strategies were significantly related with greater personal growth. Greater use of religious coping strategies was significantly related to greater grief, personal growth, and anxiety. Depression was not significantly correlated with any of the spiritual coping scales.

Hypothesis 2b. Children's grief will be related to their personal growth, anxiety, and depression after the death of a parent, grandparent, or sibling.

Pearson's product moment correlations also were used to determine the strength and direction of the relationship between grief and the outcomes of personal growth, anxiety, and depression (Table 3). Greater grief was significantly related to greater personal growth, anxiety, and depression. 
Table 3

Correlations between spiritual coping strategies and grief, personal growth, anxiety, and depression

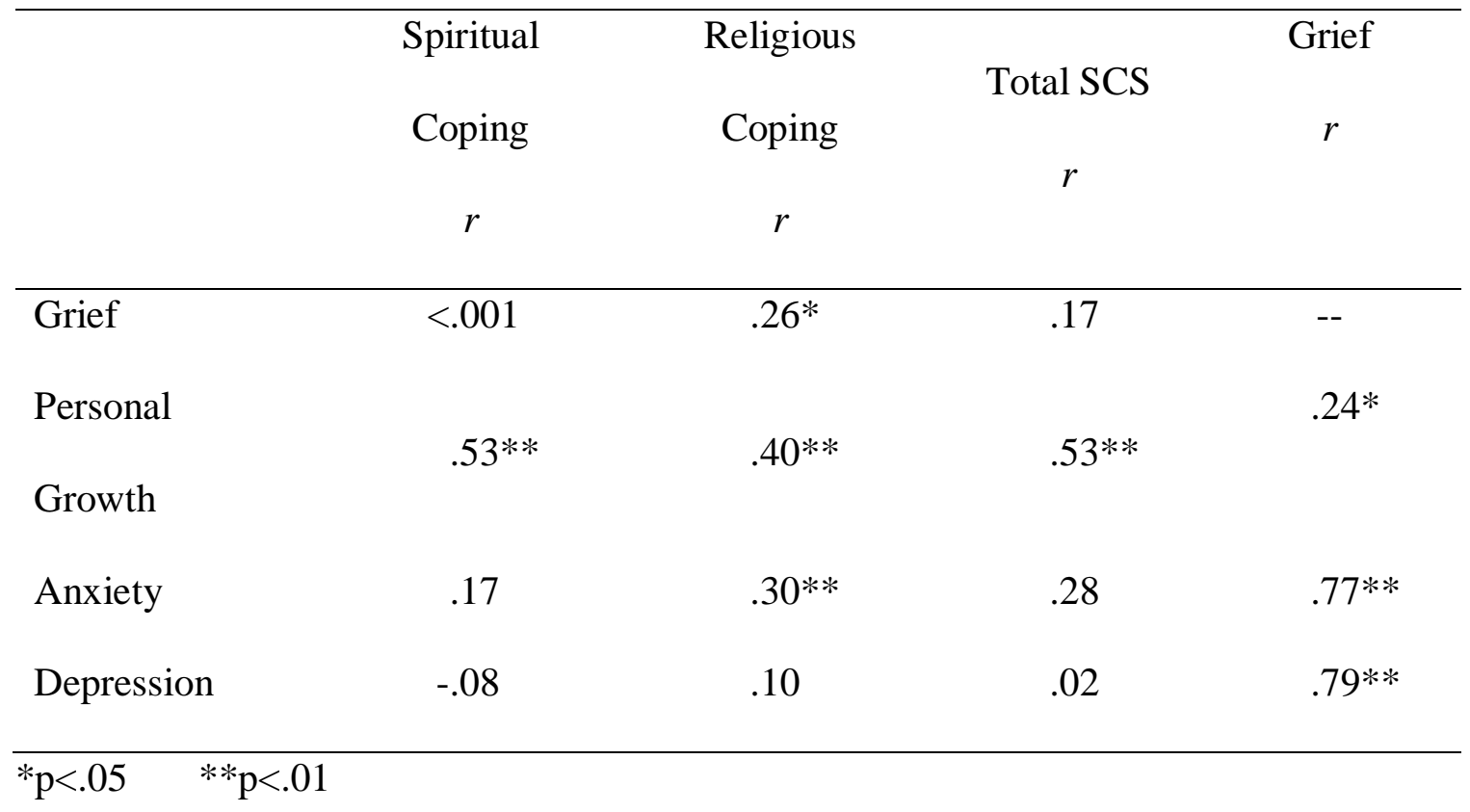

Research Question 3: Do these relationships hold after controlling for age, gender, race/ethnicity, participation in religious rituals and practices, and relationship of the deceased to the child?

Hypothesis 3a. The relationships between children's use of spiritual coping strategies and children's grief, personal growth, depression, and anxiety will hold when child's age, gender, race/ethnicity, participation in religious rituals and practices, and relationship of the deceased are controlled. 
Hierarchical multiple regression was used to address this research question. Grief and each of the 3 outcome variables (personal growth, anxiety, and depression) were regressed on child age, gender, race/ethnicity, participation in religious rituals and practices, and relationship of the deceased in the first stage, and spiritual coping and religious coping were added in the second stage. Race/ethnicity was represented by 2 dummy-coded variables, with "white" as the suppressed group. Relationship-of-thedeceased variable also was represented by 2 dummy-coded variables, with "grandparent" as the suppressed group.

The correlation between each of the independent variables was examined to determine if any of the variables were highly correlated, hence, if multicollinearity was evident (Table 4). In this study the SCSS's religious and spiritual coping subscales were used and multicollinearity can occur when one variable is a combination of other variables. Correlations of .8 or .9 are reason for concern (Pallant, 2010). The dummy coded race variables of "Hispanic" and "Black" have a high correlation. This is not surprising since they were made from the same variable. This is the same with "sibling" and "parent."

Grief. Regression equations with grief as the dependent variable were not statistically significant at both stages (Table 5).

Personal Growth. Regression equations with personal growth as the dependent variable were statistically significant at the second stage, but not at the first stage (Table 5). Greater use of spiritual coping, but not religious coping, was significantly related to greater personal growth. Explained variance in personal growth for the second stage was 
moderate. The stage two regression met the error assumptions of normal distribution and homoscedasticity.

Anxiety. Regression equations with anxiety as the dependent variable were significant at both stages (Table 5). Younger age and being Black were significantly related to greater anxiety at both stages. Religious and spiritual coping were not

Table 4

Correlations among independent variables

\begin{tabular}{|l|c|c|c|c|c|c|c|c|}
\hline Independent Variables & 1 & 2 & 3 & 4 & 5 & 6 & 7 & 8 \\
\hline 1. Age & 1.00 & & & & & & & \\
\hline 2. Gender & -.11 & 1.00 & & & & & & \\
\hline 3. Hispanic & .06 & .07 & 1.00 & & & & & \\
\hline 4. Black & -.001 & -.02 & $-.74^{* *}$ & 1.00 & & & & \\
\hline 5. Participated in rituals & $.32^{* *}$ & $-.22^{*}$ & -.10 & .07 & 1.00 & & & \\
\hline 6. Sibling & .18 & -.10 & .11 & -.11 & .06 & 1.00 & & \\
\hline 7. Parent & $-.23^{*}$ & .02 & -.02 & .01 & .04 & $-.51^{* *}$ & 1.00 & \\
\hline $\begin{array}{l}\text { 8. Religious coping } \\
\text { strategies }\end{array}$ & $-.22^{*}$ & -.09 & -.15 & $.24^{*}$ & $-.21^{*}$ & .13 & -.04 & 1.00 \\
\hline 9. Spiritual coping & & & & & & & & \\
strategies & -.03 & -.02 & .09 & -.14 & -.01 & .01 & .06 & $.45^{* *}$ \\
\hline
\end{tabular}

$* \mathrm{p}<.05 \quad * * \mathrm{p}<.01$ 
statistically significant. Explained variance in anxiety was low to moderate. The stage two regression met the error assumptions of normal distribution and homoscedasticity.

Depression. Regression equations with depression as the dependent variable were not statistically significant at both stages (Table 5). The p-p plot for the second stage suggests some non-normality in the errors. The scatterplot of the standardized predicted values and standardized residuals suggests some heteroscedasticity of the error terms. This may be due to a variable related to depression, such as anxiety, that is not in the regression equation.

Table 5

Multiple regression analyses for grief, personal growth, anxiety, and depression

\begin{tabular}{|c|c|c|c|c|c|c|c|c|}
\hline \multirow{2}{*}{$\begin{array}{l}\text { Independent } \\
\text { Variables }\end{array}$} & \multicolumn{2}{|c|}{ Grief } & \multicolumn{2}{|c|}{ Personal Growth } & \multicolumn{2}{|c|}{ Anxiety } & \multicolumn{2}{|c|}{ Depression } \\
\hline & $\beta 1$ & $\beta 2$ & $\beta 1$ & $\beta 2$ & $\beta 1$ & $\beta 2$ & $\beta 1$ & $\beta 2$ \\
\hline Age & $-.28 *$ & $-.25^{*}$ & .04 & .08 & $-.48 * *$ & $-.46 * *$ & $-.23 *$ & -.22 \\
\hline Gender & -.04 & -.01 & -.09 & -.09 & -.08 & -.08 & .03 & .04 \\
\hline Hispanic & -.03 & -.04 & .03 & .05 & .12 & .13 & -.06 & -.07 \\
\hline Black & .22 & .15 & .13 & .19 & $.35^{*}$ & $.35 *$ & .18 & .14 \\
\hline $\begin{array}{l}\text { Participated in } \\
\text { religious rituals }\end{array}$ & -.001 & .04 & -.14 & -.13 & -.01 & .002 & .07 & .09 \\
\hline Sibling & .06 & .03 & .15 & .03 & .04 & .001 & -.07 & -.07 \\
\hline Parent & .06 & .07 & .09 & -.003 & .06 & .03 & .07 & .08 \\
\hline Religious Coping & & .19 & & .09 & & .07 & & .09 \\
\hline Spiritual Coping & & -.07 & & $.56^{* *}$ & & .14 & & -.10 \\
\hline $\mathrm{F}$ & 2.08 & 1.86 & .61 & $6.13 * *$ & $5.37 * *$ & $4.73 * *$ & 1.92 & 1.55 \\
\hline
\end{tabular}




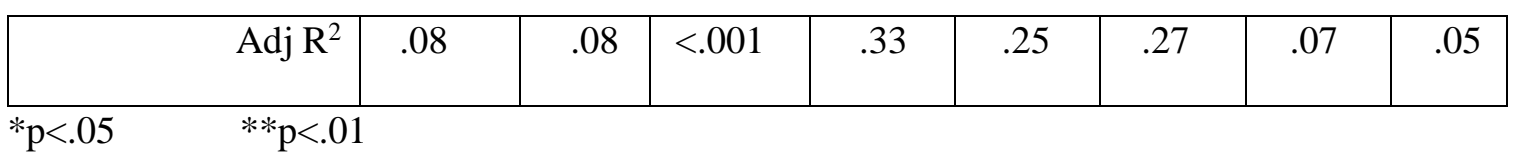

Hypothesis 3b. The relationships of children's use of spiritual coping strategies and children's grief with the 3 outcomes (personal growth, depression, and anxiety) will hold when child's age, gender, racelethnicity, participation in religious rituals and practices, and relationship of the deceased are controlled.

Hierarchical multiple regression was used to address this research question. Each of the 3 outcome variables (personal growth, anxiety, and depression) were regressed on child age, gender, race/ethnicity, participation in religious rituals and practices, and relationship of the deceased in the first stage, and spiritual coping, religious coping, and grief were added in the second stage (Table 6).

Personal Growth: Regression equations with personal growth as the dependent variable were statistically significant at the second stage, but not at the first stage (Table 6). Greater use of spiritual coping, but not religious coping, and greater grief were significantly related to greater personal growth. Explained variance in personal growth for the second stage was moderate. The stage two regression met the error assumptions of normal distribution and homoscedasticity.

Anxiety: Regression equations with anxiety as the dependent variables were significant at both stages (Table 6). Younger age and being Black were significantly related to greater anxiety at both stages. Greater use of spiritual coping, but not religious coping, and greater grief were related to greater anxiety in the second stage. Explained variance in anxiety for the second stage was high. The p-p plot for the second stage suggests some minor non-normality in the errors. The scatterplot of the standardized 
predicted values and standardized residuals supports the assumption of homoscedasticity of the error terms.

Depression: Regression equations with depression as the dependent variable were significant at the second stage (Table 6). Greater grief was significantly related to greater depression. Explained variance in depression for the second stage was moderate. The p-p plot after the second stage suggests some non-normality in the errors. The scatterplot of the standardized predicted values and standardized residuals supports the assumption of homoscedasticity of the error terms. This may be due to a variable related to depression, such as anxiety, that is not in the regression equation.

Table 6

Multiple regression analyses for the outcomes of personal growth, anxiety, and depression

\begin{tabular}{|l|c|c|c|c|c|c|}
\hline \multirow{2}{*}{ Independent Variables } & \multicolumn{2}{|c|}{ Personal Growth } & \multicolumn{2}{c|}{ Anxiety } & \multicolumn{2}{c|}{ Depression } \\
\cline { 2 - 7 } & $\beta 1$ & $\beta 2$ & $\beta 1$ & $\beta 2$ & $\beta 1$ & $\beta 2$ \\
\hline Age & .05 & .14 & $-.47^{* *}$ & $-.28^{* *}$ & $-.23^{*}$ & -.02 \\
\hline Gender & -.09 & -.09 & -.09 & -.09 & .03 & .05 \\
\hline Hispanic & .03 & .06 & .12 & .16 & -.06 & -.03 \\
\hline Black & .13 & .16 & $.35^{* *}$ & $.26^{* *}$ & .18 & .02 \\
\hline $\begin{array}{l}\text { Participated in religious } \\
\text { rituals }\end{array}$ & -.15 & -.14 & -.02 & -.04 & .07 & .06 \\
\hline Sibling & .15 & .02 & .04 & -.04 & -.07 & -.09 \\
\hline Parent & .09 & -.01 & .05 & -.04 & .07 & .03 \\
\hline Religious Coping & & .04 & & -.09 & & -.07 \\
\hline
\end{tabular}




\begin{tabular}{|c|c|c|c|c|c|c|}
\hline Spiritual Coping & & $.57 * *$ & & $.21 * *$ & & -.04 \\
\hline Grief & & $.22 *$ & & $.69 * *$ & & $.80 * *$ \\
\hline $\mathrm{F}$ & .61 & $6.39 * *$ & $5.28 * *$ & $21.90 * *$ & 1.92 & $17.41 * *$ \\
\hline $\operatorname{Adj} R^{2}$ & $<.001$ & .37 & .25 & 69 & .07 & .64 \\
\hline
\end{tabular}

$* \mathrm{p}<.05 \quad * * \mathrm{p}<.01$

\section{CHAPTER V}

\section{DISCUSSION}

The CDC (2016) reported a total of 23,215 deaths of infants under one year of age; annual death rates of 3,830 per 100,000 children 1 to 4 years, 5,250 per 100,000 children 5 to 14 years, and 9,586 per 100,000 adolescents 15 to 19 years. This large number of child deaths each year also affects a larger number of siblings (Fletcher, Mailick, Song, \& Wolfe, 2013). This supports the need for research on children's responses to sibling death. The CDC (2016) also reported a total of 2,626,418 adult deaths. Parent and grandparent deaths can be accounted for within this high adult mortality rate. The majority of the literature focuses on the effects that children's deaths have on parents, failing to recognize the emotional, psychological, and behavioral grieving responses that a parent's, grandparent's, or sibling's death has on surviving children. 
The death of a sibling signifies the loss of a role model, friend, confidante, and playmate (Woodgate, 2006). Bereaved siblings are at risk for developing mental health disorders, such as depression, prolonged grief disorder (PGD), and post-traumatic stress disorder (PTSD) (Barrera et al., 2013; Martinson \& Campos, 1991). The impact of a parent's death for dependent children can include decreased economic resources, change in residence, loss of contact with friends and neighbors, change in caretakers, and loss of time with the surviving parent (Wolchik et al., 2006). Parentally bereaved children not only face the permanent physical absence of their parent but they also may have been exposed to traumatic images and situations associated with the end of the parent's life, thereby increasing the likelihood of developing PTSD (Kaplow et al., 2014). Research on the effects that the death of a grandparent has on the child is limited. Research has found that religious and spiritual forms of coping are used by children when they are facing stress, trauma, or illness, and they are important in promoting positive posttraumatic growth (Bryant-Davis et al., 2012).

In studies of children's religious beliefs and practices, children's beliefs were not obtained directly from the children, but from parents' reports of their children's beliefs (Neuman, 2011; Schottenbauer et al., 2007). Children's reports of their beliefs and practices would be a more valid measure. Potential issues and problems associated with gender and individual differences in the spiritual development of young children need to be explored since males and females are generally perceived differently and often based on the individual's personal experiences (Eaude, 2004). The current research sheds light on gender differences in children's use of spiritual activities during bereavement. 
Most of the studies pertaining to child and adolescent bereavement have concentrated on the death of a parent, not a grandparent or sibling (Sood et al., 2006). Only the study by Ens and Bond (2005) examined the relationships between adolescent bereavement following the death of a grandparent. Research on the spiritual coping strategies used by children after the death of a parent, grandparent, or sibling is limited.

The majority of the research on children's bereavement focuses on siblings and parents who died of cancer excluding other causes of death such as suicide, homicide, heart attack, stroke, genetic conditions, and car crashes. Most of the participants in these studies were Protestant and Caucasian. This diminishes the generalizability of results to families who practice other religions and who are from different cultures. Also, most of the studies vary with respect to elapsed time after the death, which may have an effect on the child's grief, personal growth, anxiety, and depression. Methodological dissimilarities such as timing of assessments, age of participants, and instruments used makes it difficult to pinpoint specific grieving patterns in children (Barrera et al., 2013).

The current literature does not describe the spiritual coping strategies children use after the death of a parent or sibling. Research on the effects of a grandparent's death on children and the effectiveness of spiritual coping strategies used by children after such a loss is limited to non-existent. There is also scant research regarding children's participation in religious rituals and practices after the death of a parent or sibling, and none after the death of a grandparent.

The purpose of this correlational study was to identify 1) differences in the spiritual coping strategies used by children across age groups, gender, race/ethnicity, participation in religious rituals and practices, and the relationship of the deceased to the 
child (parent, grandparent or sibling), and 2) the relationship between children's spiritual coping strategies and grief, personal growth, anxiety, and depression after the death with and without controls for child's age, gender, race/ethnicity, participation in religious rituals and practices, and relationship to the deceased.

\section{Study overview}

The sample consisted of 97 children ( 8 to 18 years old, able to read and write English, and in age appropriate school grade level +/- 1 year) who had experienced loss of a parent $(n=39)$, grandparent $(n=31)$, or sibling $(n=27)$ in the past 2 years. Children who could not participate verbally, were living in foster care, had a history of psychiatric conditions, or had more than one family member die in the previous 2 years were excluded. The child's parent/legal guardian had to be at least 18 years old and able to read English and/or Spanish. Most children were school age, Hispanic, and participated in religious rituals around the time of their parent's, grandparent's, or sibling's death; about half were boys. Comparison of the three relationship-of-the-deceased groups showed no statistical differences across groups on child age, gender, race/ethnicity, and participation in rituals.

Parents completed a demographic information form while their child completed their questionnaires in a separate part of the house. The children completed the Spiritual Coping Strategies Scale (SCSS), Hogan's Inventory of Bereavement (HIB), Spence's Child Anxiety Scale (SCAS), and the Child Depression Inventory (CDI). The PI and/or the RA read the items to the child to reduce the time for the interview as well as the effect of the child's reading ability on their responses.

Findings for each of the research questions are described below. 


\section{Differences in spiritual coping by person factors}

Significant differences on religious coping were found between children who did and did not participate in religious rituals around the time of the death. Children who participated in religious rituals used less religious coping strategies than children who did not participate. Perhaps this could be because religious coping occurs over time and religious rituals are done soon after the loved one dies. Benore et al. (2008) studied the relationship between religious coping strategies and adjustment in 87 primarily African American and Baptist children and adolescents (ages 8-17) hospitalized for asthma. Contrary to most studies, greater religious coping was related to poorer adjustment. Any positive effects of religious coping may be evident at a later time. More than half of the children in this study experienced the death of their loved one within a period of 11 months or less. Timing of this study's data collection may be too soon to see the positive outcomes of religious coping or positive outcomes of religious coping may not exist in some circumstances.

It may also be that children who attended the religious rituals did not need to use religious coping strategies because the rituals helped them deal with their grief and loss. A study by Softing, Dyregrov, and Dyregrov (2016) examined how Norwegian children were included in death related rituals after the death of a parent or sibling, their experience with their own participation, and the meaning that rituals had for them. These children felt that, by participating in the death rituals, they were able to "see for themselves" which helped them accept the reality of the loss and they were also able to say goodbye to the deceased. Children who did not attend the religious rituals may have needed to use more religious coping strategies to accept and make sense of the loss. In a 
study by Brooten et al. (2015), some families restricted the attendance of pregnant women and small children in wakes and funerals, perhaps for cultural reasons. The scant literature on death rituals concentrates mostly on adult deaths and the adults who experience them. Death rituals help mourners legitimize their grief, acknowledge the death, and maintain a connection with the deceased (Gudmundsdottir \& Chesla, 2006; Weeks, 2004).

In this study, significant differences in spiritual coping strategies were not found between children by age group, gender, race/ethnicity, and the relationship of the deceased to the child. Baldacchino et al. (2013) identified commonalities across cultures in the spiritual coping of patients with chronic illnesses receiving rehabilitation services in Malta and Norway. Even though spirituality is distinct and personal to each individual, it is believed to be universal to all human beings, regardless of age or stage of development (Kenny, 1999). Spiritual coping may provide a way for children and adolescents to make sense of a traumatic experience (Bryant-Davis et al., 2012). Hay (2003) argues that all children have spirituality and are more in touch with their spirituality than adults. Children may employ religious coping in crisis situations for its protective factors and to gain a deeper understanding and make sense of the negative experience. It may be that children's use of religious coping strategies is similar regardless of their relationship to the deceased family member.

\section{Spiritual coping strategies, grief, and outcomes}

In this study greater use of spiritual coping strategies overall and the specific spiritual and religious coping strategies was significantly related to greater personal growth. Religious and spiritual coping strategies are used by children when they are 
facing stress, trauma, or illness (Bryant-Davis et al., 2012). Religion gives meaning to situations and life events that humans fear and don't comprehend. It serves as a path for human behavior and thinking, making it possible to find meaning in situations that are difficult to understand (Bryant-Davis et al., 2012). Children also use spirituality to shape their perceptions of life (Sifers, Warren, \& Jackson, 2012). Research with adults and adolescents finds that spirituality is part of their overall health, decreasing delinquency and psychopathology, and contributing to coping and resiliency (Koenig, 2004; Pendleton, et al., 2002) which in turn enhances personal growth.

Spirituality and religion can provide meaning within the circumstances involving a specific person-environment transaction (Park \& Folkman, 1997). In a transactional model of coping, meaning making is conceptualized as a process of cognitive reassessment essential to successfully adapt under circumstances that are chronic or not easily improved by coping efforts (Park \& Folkman, 1997).

According to Good et al. (2011), religion is a multidimensional construct that pertains to institutional religious expressions such as dogma and ritual, as well as personal religious expressions such as spirituality, beliefs, and religious practices. Spirituality is different from religion because it is not based on a discrete value system (Farrell et al., 2008). In contrast, others argue that spirituality and religiosity are highly intertwined despite their distinct characteristics (Good et al., 2011; Walker, Reid, O’Neill, \& Brown, 2009).

In this study greater grief was significantly related to greater personal growth, anxiety, and depression. Bereavement (defined as having a loved one die) and grief (defined as the reaction to the death of a loved one) are two concepts not easily 
understood during childhood and adolescence, and only few longitudinal studies have been reported (Wilkins \& Woodgate, 2005; Woodgate, 2006). Research with bereaved children and adults finds more grief reactions and distress for deaths which were more recent (within 1-1.5 years), unexpected, in the hospital versus the home, and associated with prolonged illness and/or suffering (Barry, Kasl, \& Prigerson, 2002; Gamino, Sewell, \& Easterling, 2000; Seecharan, Andresen, Norris, \& Toce, 2004). Oltjenbruns (2001) posits that "childhood development is ongoing; grief and feelings of loss are often repeatedly processed as children become more capable of understanding their loss" ( $p$. 73). Children manifest their grief differently, and duration of their grief responses may be underestimated by adults. Children tend to grieve intermittently for several years (Aiken, 2001). Grief can be prolonged and can resurface throughout the child's life when they examine the death from their evolving and maturing developmental perspective.

Circumstances such as unnatural death, close emotional and biological ties to the deceased (parents, siblings, close friends), and the child's age may affect a child's response to the death (Rostila \& Saarela, 2011). The death of a loved one in childhood and adolescence is associated with increased emotional problems which include depression, anxiety and posttraumatic stress, as well as somatic complaints and behavioral problems ( Dowdney, 2008; Kaplow, Layne, Pynoos, Cohen, \& Lieberman, 2012; Kaufman \& Kaufman, 2006). Bereaved children may not find the emotional support they need from their parents who are focused on their own pain (deCinque et al., 2006), and some children may not seek support or talk about their reactions thinking that they have to protect their grieving parents (Dyregrov \& Dyregrov, 2005; Youngblut \& 
Brooten, 2013). Surviving children are often seen as "forgotten grievers" since their loss is insufficiently acknowledged (Crehan, 2004; Horsley \& Patterson, 2006).

Greater grief was related to greater personal growth; perhaps the children in this study relied on continuing bonds to cope with the death of their loved one which, in turn, increased their personal growth. Surviving children often share a strong connection or bond with a deceased sibling that can surpass death (Packman, Horsley, Davies, \& Kramer, 2006). In a study by Youngblut and Brooten (2013), parents described children's responses 7 months after their siblings died in the NICU or PICU. Parents commented about their children "maintaining a connection with their deceased sibling" which included wearing t-shirts with the sibling's picture, talking to their sibling, and looking at books and pictures that the sibling found important. Sometimes children kept strong bonds with their deceased siblings by wearing their clothes, playing with their toys, visiting their favorite places, eating their favorite foods, or listening to their favorite songs (Foster et al., 2011). Children also may feel connected to their deceased brother or sister through their religious beliefs, including being reunited with their brother or sister in heaven or the afterlife, which serves as a continuing bond (Forward \& Garlie, 2003). For grieving children keeping a strong bond with their brother or sister may help them cope with the loss which may enhance personal growth. Research that only focuses on the negative outcomes of grief fails to acknowledge the distinctive human capacity to cope with hardship and ignores grief's power to act as an agent for positive change and growth (Hogan, Greenfield, \& Schmidt, 2001).

In a study of 18 Korean young adult children aged 20-24 years after the death of a parent, Yang (2012) found that continuing bonds with the deceased parents appeared to 
help with the children's psychological functioning. Adolescents reported a growth in spirituality. Yang posits that children may use continuing bonds "to overcome the shock of the parental death, to deal with previous conflicts with their deceased parent, or to settle unsolved family business" (p. 8).

\section{Relationship with control for person factors}

In this study, greater use of spiritual coping, but not religious coping, was significantly related to greater personal growth. Although religious coping was not a predictor of personal growth in this study, other research studies show that individuals use religious and spiritual coping to find meaning and decrease stress when facing difficult situations and life experiences (Siegel \& Schrimshaw, 2002). Individuals who used positive religious coping methods had more positive health outcomes than people who used negative religious coping (difficult situations are God's punishment) (Pargament, 2001).

Spirituality is characterized as a complex, multidimensional construct apparent in an individual's beliefs, behavior, and experience (Miller \& Thoresen, 1999). The Spiritual Framework of Coping is phenomenological, dynamic and relational, and process-oriented. Spiritual appraisals and spiritual coping behaviors function as mediating factors in the process of coping with stress. Spiritual appraisals have to do with making sense of the stressor based on one's spiritual beliefs. An individual can attempt to make meaning out of the situation by attributing it to God's will or God's plan. When an individual attempts to find meaning in a situation, initial levels of distress may be reduced (Davis, Nolen-Hoeksema, \& Larson, 1998) to a low enough level to facilitate engagement in coping behavior. Spiritual coping encompasses particular behaviors that 
an individual uses to respond to the stressor (problem focused) or related emotional reactions (emotion focused). In this framework, spiritual person factors function as a contextual framework that makes it feasible for the individual to formulate his or her interpretation, comprehension, and reaction to life experiences (Krok \& Dariusz, 2008; Tuck, Alleyne, \& Thinganjana, 2006). Such beliefs may assist the individual by helping him or her create meaning out of his or her suffering therefore creating a more hopeful attitude (Garssen, Uwland-Sikkema, \& Visser, 2015). Children in this study may have used spiritual coping to help them create meaning after the death of their loved one which may have increased their personal growth.

Younger age and being Black, but not religious and spiritual coping, were significantly related to greater anxiety. Research in child bereavement has described an array of grieving responses: emotional (depression, sadness, anxiety, feelings of guilt), behavioral (sleep disturbances, acting out), social (withdrawal, loneliness), and deterioration in school performance (Fanos et al., 2009). Younger children may have more anxiety than older children because of their lack of understanding of death due to their developmental level. As children's development progresses with age, so does their understanding of death (Willis, 2002).

Also, younger children depend more on their parents for emotional support than older children. After the death of a close family member, parents may not be able to provide the emotional support their children need to curb their anxiety because of the parent's own pain (DeCinque et al., 2006) which may explain why younger children have higher anxiety levels. Also, parents may leave younger children out of discussions and activities surrounding the loved one's death, thinking they are protecting the children, and 
perhaps inadvertently providing children with less support and information. Adolescents are more likely to have close relationships with peers and be able to share their feelings with their friends in lieu of their parents. Peer relationships become increasingly important in adolescence (Geckova, Pudelsky, \& Tuinstra, 2000). Adolescents may have lower anxiety levels than younger children because of the emotional support they receive from their social circle.

In this study, Black children had more anxiety than White children. A longitudinal study by Grover, Ginsburg, and Ialongo (2005) examined the relationship between several types of predictors and symptoms of anxiety in first grade and again 6 years later in high risk African-American children. The risk factors were grouped into six domains (i.e., Loss-Death, Loss-Separation, Social Adversity, Negative Family Environment, Academic Difficulties, and Peer Rejection). Results showed that children who experienced a more negative family environment, had a greater number of losses and deaths, and experienced academic failures in the first grade exhibited higher levels of anxiety (initially and/or at the six year follow up). It may be that Black children in this study had increased anxiety compared to White children since they were exposed to familial and environmental risk factors.

Gray, Carter, and Silverman (2011) conducted a cross-sectional study that explored the relationships between children's ethnic pride, perceived parenting behavior, anxiety sensitivity, and child anxiety symptoms in 266 African American school children. Results of the study showed that children with perceptions of high parental control (low level of encouragement of children's independence) reported high anxiety sensitivity. 
Black children in this study may be affected by high parental control which is a factor that is related to higher anxiety levels in African American children.

\section{Effects of spiritual coping strategies and grief on outcomes for person factors}

In this study greater use of spiritual coping, but not religious coping, and greater grief were significantly related to personal growth. Use of spiritual coping had a strong effect on personal growth regardless of whether grief was included in the model. These findings support the conceptual framework for personal growth.

In this study, younger age and being Black were significantly related to greater anxiety at both stages. These effects were weaker, but still significant, when religious and spiritual coping and grief were added as independent variables in the regression model. This suggests that grief mediates some of the effect of age and being Black on anxiety, but not all of it. Younger age and being Black was related to greater anxiety in children with and without the mediating effect of grief.

Greater use of spiritual coping, but not religious coping, and greater grief were related to greater anxiety, but only when grief was included in the regression model. Before grief was added, the effects of spiritual and religious coping on anxiety were small and not statistically significant. When grief was the dependent variable the effects of religious and spiritual coping on grief were small and not statistically significant.

In a study of 1,296 individuals facing stressful situations and 352 individuals undergoing alcohol addiction therapy, Charzyn'ska (2014) found that greater use of negative spiritual coping was related to poorer mental and physical functioning. Negative spiritual coping prevents an individual from drawing strength from spiritual resources; it prevents the quest for sense and meaning in life, and hinders its growth (Charzynska, 
2014). Children in this study may have used negative spiritual coping, which could have prevented them from using spiritual resources to find sense and meaning after their loved one's death which may have increased their anxiety.

In this study greater grief was significantly related to greater depression with and without control for all other independent variables (age, gender, race/ethnicity, participation in religious rituals, relationship-to-deceased group, and spiritual and religious coping). Relationships between depression and these independent variables were small and not statistically significant in all regression models where depression was the dependent variable.

\section{Summary of findings}

This correlational study identified differences in the spiritual coping strategies used by children across age groups, gender, race/ethnicity, participation in religious rituals and practices, and the relationship of the deceased to the child (parent, grandparent or sibling), and the relationship between children's use of spiritual coping strategies and grief, personal growth, anxiety, and depression after the death, with and without controls for child's age, gender, race/ethnicity, participation in religious rituals and practices, and relationship of the deceased to the child.

Children who participated in religious rituals after the death used less religious coping strategies than children who did not participate. When child's age, gender, race/ethnicity, participation in religious rituals and practices, and relationship of the deceased were controlled, greater use of spiritual coping, but not religious coping, and 
greater grief were significantly related to greater personal growth and greater anxiety. Younger children and Black children had significantly greater anxiety. Only grief was significantly related to depression. Children who experienced the death of a parent, grandparent, or sibling had similar outcomes. The results of this study will provide insight regarding the effects of children's spiritual coping after parent, grandparent, or sibling death on their grief, personal growth, and mental health which is a subject area that has not been widely studied and very little is known about.

\section{Limitations}

A few limitations are present in this study. Even though this study is multicultural and multiethnic and included children of all religions, children were predominantly Hispanic and Catholic. Although being Black rather than White was a significant predictor of anxiety, the smaller number of children in these two racial groups limits the study's generalizability to Black children and White children and to families who are from different cultures and religions. This is the first study to use the SCSS with children. Although the total scale and subscales showed good internal consistency, the results of this study raise questions about its validity as a measure of religious and spiritual coping in children. The activities in the items of the spiritual coping strategies scale may need revision to make them more applicable to children, e.g. including activities like playing

videogames. In response to the item about reading books, adults might include paper and electronic forms in their definition of a "book," but children may consider only the paper form as a "book."

More than half of the children in this study had experienced their loved one's death within the previous 11 months or less. Results may differ in populations were the 
loss happened more than 11 months ago. Also, children who suffered the death of their loved one less than 12 months ago may have been more affected and therefore were more likely to attend bereavement camps and support groups. Results may differ if children were recruited from other settings.

\section{Implications for practice and education}

The findings of this research are applicable to nursing practice and education. Results of this multicultural/multiethnic study on children's use of spiritual coping strategies identified activities or coping strategies that children find helpful in dealing with a loved one's death. Additionally, the findings that younger children may experience

more anxiety and possibly grief and depression than older children is in direct contrast to what the general public may think. People often think that younger children will forget about the death and be less affected. Black children experienced more anxiety than White children. Both provide evidence of cultural differences in the death of a loved one and differences due to children's age and development.

Bringing awareness of the positive effects of children's use of spiritual coping strategies for their personal growth can be done through continuing education courses available to healthcare personnel in the hospital. Also, this information should be disseminated via community education courses to healthcare providers and grief counselors in the community. Academic courses in both nursing and medicine should incorporate these research findings. Learning how children's spiritual coping strategies increases their personal growth as well as the age and racial differences that exist with respect to grief and anxiety in bereft children will enhance the student's knowledge base 
with respect to the grieving process in children. Once these students graduate they will be better prepared to help children that have undergone the loss of a loved one.

\section{Implications for research}

Findings from this study have significant implications for future research.

Children in this study had experienced the death of a parent, grandparent, brother, or sister within the past two years. Further research is needed to determine if children's spiritual coping strategies remain related to greater personal growth more than two years after the death. Also, future research is needed to determine if younger age and being Black are related to greater anxiety with a different sample of children after the death of a parent, grandparent, brother, or sister. Recruiting children from a variety of sources, especially those not initiated by the parent or guardian (like bereavement camp or support groups), may yield different results.

In a study by Servaty-Seib and Pistole (2006) when emotional closeness to the deceased person was taken into account, grandchildren who had a close relationship with their grandparent had higher grief scores than children who did not, with levels that resembled those from other types of loss. Future research should consider how close the child's or adolescent's relationship had been with their grandparent. This can provide valuable information regarding children's spiritual coping strategies after the loss of a grandparent when compared to parental and sibling loss. 


\section{References}

Aiken, L. R. (2001). Dying, death, and bereavement. Mahwah, NJ: Lawrence Erlbaum.

Abu-Rabia, A., \& Khalil, N. (2012). Mourning Palestine. Anthropology of the Middle East,7(2), 1-18.

American Psychiatric Association (2000). Diagnostic and statistical manual of mental disorders ( $4^{\text {th }}$ ed. Text Revision). Washington, DC: Author.

Asai, M., Fujimori, M., Akisuki, N., Inagaki, M., Matsui, Y., \& Uchitomi, Y. (2010). Psychological states and coping strategies after bereavement among the spouses of cancer patients: A qualitative study. Psycho-Oncology, 19, 38-45.

Baldacchino, D. R., Torskenaes, K., Kalfoss, M., Borg, J., Tonna, A., Debattista, C., Decelis, N., and Mifsud, R. (2013). Spiritual coping in rehabilitation- a comparative study: part 1. British Journal of Nursing, 22(4), 228-232.

Baldacchino, D. R., \& Buhagiar (2003). Psychometric evaluation of the spiritual coping strategies scale in English, Maltese, back-translation and bilingual versions. Journal of Advanced Nursing, 42(6), 558-570. 
Bamford, C., \& Lagattuta, H. (2010). A new look at children's understanding of mind and emotion: The case of prayer. Developmental Psychology, 46(1), 78-92.

Barnes, L. L., Plotnikoff, G. A., Fox, K., \& Pendleton, S. (2000). Spirituality, religion and pediatrics: Intersecting worlds of healing. Pediatrics, 104(6), 899-908.

Barrera, M., Alam, R., Mammone D’Agostino, N., Nicholas, D. B., \& Schneiderman, G. (2013). Parental perceptions of siblings' grieving after childhood cancer death: A longitudinal study. Death Studies, 37, 25-46.

Barrera, M., O’Connor, K., D’Agostino, N. M., Spencer, L., Nicholas, D., Jovcevska, V., Tallett, S., \& Schneiderman, G. (2009). Early parental adjustment and bereavement after childhood cancer death. Death Studies, 33, 1-24.

Barry, L. C., Kasl, S. V., \& Prigerson, H. G. (2002). Psychiatric disorders among bereaved persons: the role of perceived circumstances of death and preparedness for death. American Journal of Geriatric Psychiatry, 10, 447-457.

Batten, M., \& Oltjenbruns, K. (1999). Adolescent sibling bereavement as a catalyst for Spiritual development: A model for understanding. Death Studies, 6, 529-546.

Benore, E., Pargament, K. I., Pendleton, S. (2008). An initial examination of religious coping in children with asthma. The International Journal for the Psychology of Religion, 18, 267-290.

Bernstein, K., D’Angelo, L. J., \& Lyon, M. E. (2012). An exploratory study of HIV+ adolescent's spirituality: Will you pray with me? Journal of Religious Health, 52(4), 1253-1266. doi:10.1007/s10943012-9565-1

Bering, J. M., Hernandez-Blasi, C., \& Bjorklund, D. F. (2005). The development of "afterlife" beliefs in religiously and secularly schooled children. British Journal of Developmental Psychology, 23, 587-607.

Boelen, P. A., \& Van Den Bout, J. (2005). Complicated grief, depression, and anxiety as distinct post-loss syndromes: a confirmatory factor analysis study. American Journal of Psychiatry, 162, 2175-2177.

Boer \& Zock (2004). Dreams of passage: An object-relational perspective on a case of a Hindu death ritual. Religion, 34, 1-14.

Bolton, C., \& Camp, D. J. (1989). The post-funeral ritual in bereavement counseling and grief work. Journal of Gerontological Social Work, 13(3), 49-59.

Bonoti, F., Leondari, A., \& Mastora, A. (2013). Exploring children's understanding of 
death:Through drawings and the death concept questionnaire. Death Studies, 37, 47-60.

Bosacki, S., Elliott, A., Akseer, S., \& Bajovic, M. (2010). The spiritual component of children's lives: the role of media. British Journal of Religious Education, 32(1), 49-61.

Boylan, L. N. (2005). Caring for patients of diverse religious traditions: Judaism. Home Healthcare Nurse, 23(12), 794-797.

Boynton, H. M. (2011). Children's spirituality: epistemology and theory from various helping professions. International Journal of Children's Spirituality, 16(2), 109127.

Braswell, G. S., Rosengren, K. S., \& Berenbaum, H. (2012). Gravity, God, and ghosts? Parents beliefs in science, religion, and the paranormal and the encouragement of beliefs in their children. International Journal of Behavioral Development, 36(2), 99-106.

Brent, D., Melhem, N., Donohoe, M. B., \& Walker, M. (2009). The incidence and course of depression in bereaved youth 21 months after the loss of a parent to suicide, accident, or sudden natural death. American Journal of Psychiatry, 166(7), 786794.

Brent, D. A., Melhem, M. N., Masten, A. S., Porta, G., \& Walker-Payne, M. (2012). Longitudinal effects of parental bereavement on adolescent developmental competence. Journal of Clinical Child and Adolescent Psychology, 41(6), 778791.

Brooten, D., Youngblut, J., Charles, D., Roche, R., Hidalgo, I., \& Malkawi, F. (2015). Death rituals reported by White, Black, and Hispanic Parents following the ICU death of an infant or child. Journal of Pediatric Nursing, 31, 132-140.

Bryan, E. (2003). The impact of multiple preterm births on the family. International Journal of Obstetrics and Gynecology, 110, 24-28.

Bryant-Davis, T. (2005). Coping strategies of African American adult survivors of childhood violence. Professional Psychology: Research and Practice, 36, 409414.

Bryant-Davis, T., Ellis, M. U., Burke-Maynard, E. Moon, N., Counts, P. A., \& Anderson, G. (2012). Religiosity, spirituality, and trauma recovery in the lives of children and adolescents. Professional Psychology, 4(4), 306-314. 
Burg, S., Mayers, R. A., \& Miller, L. J. (2011). Spirituality, religion, and healthy development in adolescents. Encyclopedia of Adolescence, 1, pages. doi: 10.1016/B978-0-12-373915- 5.00042-5

Burrell, B. (1996). Nine night: Death and dying in Jamaica. American Visions, 2(5), 2527.

Carleton, R., Esparza, P., Thaxter, P., \& Grant, K. (2008). Stress, religious coping resources, and Depressive symptoms in an urban adolescent sample. Journal for the Scientific Study of Religion, 47(1), 113-121.

Centers for Disease Control (2016). Retrieved on May 25, 2017 from https://www.cdc.gov/nchs/data/nvsr/nvsr65/nvsr65_04.pdf

Centers for Disease Control (2016). Retrieved on May 24, 2017 from https://www.cdc.gov/nchs/data/databriefs/db229.pdf

Chan, C. L., \& Mak, J. M. (2000). Benefits and drawbacks of Chinese rituals surrounding care for the dying. In R. Fielding and C. L. Chan (Eds.), Psychological oncology and palliative care in Hong Kong: The first decade (pp. 255-70). Hong Kong: Hong Kong University Press.

Charzyn'ska, E. (2015). Multidimensional approach toward spiritual coping: construction and validation of the spiritual coping questionnaire (SCQ). Journal of Religious Health, 54, 1629-1646.

Christ, G. H. (2000). Impact of development on children's mourning. The Cancer Practice, 8(2), 72-81.

Clements, P. T., Vigil, G. J., Manno, M. S., Henry, G. C., Wilks, J., Das, S., et al. (2003). Cultural perspectives of death, grief, and bereavement. Journal of Psychological Nursing, 41(7), 18-26.

Cohen, J. A., Mannarino, A. P., \& Staron, V. R. (2006). A pilot study of modified cognitive-behavioral therapy for childhood traumatic grief (CBT-CTG). Journal of the Adolescent Academy of Child and Adolescent Psychiatry, 45(12), 14651473.

Corwyn, E., \& Benda, B. 2000. Religiosity and church attendance: The effects on use of "hard drugs" controlling for sociodemographic and theoretical factors. International Journal for the Psychology of Religion, 10, (241-58).

Cotton, S., Grossoehme, D., \& McGrady, M. E. (2012). Religious coping and the use of prayer in children with sickle cell disease. Pediatric Blood Cancer, 58, 244-249.

Cotton, S., Kudel, I., Roberts, I. H., Pallerla, H., Tsevat, J., Succop, P., \& Yi, M. S. 
(2009). Spiritual well-being and mental health outcomes in adolescents with or without inflammatory bowel disease. Journal of Adolescent Health, 44, 485-492.

Cotton, S., Larkin, E., Hoopes, A., Cromer, B. A., \& Rosenthal, S. L. (2005). The impact of adolescent spirituality on depressive symptoms and health risk behaviors. Journal of Adolescent Health, 36(6), 529-536.

Cotton, S., Zebracki, K., Rosenthal, S. L., Tsevat, J., \& Drotar, D. (2006). Religion, spirituality, and adolescent health outcomes: a review. Journal of Adolescent Health, 38, 472-480.

Crehan, G. (2004). The surviving sibling: The effects of sibling death in childhood. Psychoanalytic Psychotherapy, 18(2), 202-219.

Davis, C. G., Nolen-Hoeksma, S., \& Larson, J. (1998). Making sense of loss and benefitting from the experience: Two construals of meaning. Journal of Personality and Social Psychology, 75(2), 561-574.

DeCinque, N., Monterosso, N., Dadd, L., Siddhu, G., Macpherson, R., Aoun, S. Bereavement support for families following the death of a child from cancer: experience of bereaved parents (2006). Journal of Psychosocial Oncology, 24(2), 65-84.

Dew, R. E., Daniel, S. S., Goldston, D. B., McCall, W. V., Kuchibhatla, M. Schleifer, C., et al. (2010). A prospective study of religion/spirituality and depressive symptoms among adolescent psychiatric patients. Journal of Affective Disorders, 120, 49-157. doi: 10.1016/j.jad.2009.04.029

Dillen, L., Fontaine, J. R. J., \& Verhofstadt-Deneve, L. (2009). Confirming the distinctiveness of complicated grief from depression and anxiety among adolescents. Death Studies, 33, 437-461.

Dimond, B. (2004). Disposal and preparation of the body: Different religious practices. British Journal of Nursing, 13(9), 547-549.

Doran, G., \& Hansen, N. (2006). Constructions of Mexican-American family grief after the death of a child: An exploratory study. Cultural Diversity and Ethnic Minority Psychology, 12, 199-211.

Dowdney, L. (2008). Children bereaved by parent or sibling death. Normal and Abnormal Development, 796, 270-275.

Dyregrov, K., \& Dyregrov, A. (2005). Siblings after suicide-“The forgotten bereaved." Suicide Life Threat, 35(6), 714-724. 
Dyregrov, A., \& Yule, W. (1995, May). Screening measures: The development of the UNICEF screening battery. Paper presented at the Fourth European Conference on Traumatic stress, Paris, France.

Eaude, T. 2004. Do young boys and girls have distinct and different approaches and needs in relation to spiritual development? International Journal of Children's Spirituality, 9(1), p. 53-66.

Elliot, A., Bosacki, S., Woloshyn, V., \& Richards, M. (2002). Exploring pre-adolescents' media and literacy choice. Language and Literacy: A Canadian Educational E Journal, 3, 1-13.

Emmons, R. A. (1999). The psychology of ultimate concerns: Motivation and spirituality in personality. New York: The Guilford Press.

Ens, C. \& Bond, J. (2005). Death anxiety and personal growth in adolescents experiencing death of a grandparent. Death Studies (29), 171-178.

Epstein, R., Kalus, C., \& Berger, M. (2006). The continuing bonds of the bereaved towards the deceased and adjustment to loss. Mortality, 11, 253-269.

Fanos, J, H., Little, G. A., \& Edwards, W. H. (2009). Candles in the snow: Ritual and memory for siblings of infants who died in the intensive care nursery. The Journal of Pediatrics, 154, 849-853.

Farrell, J., Brooks, S., Cooper, J. H. Mathias, L. (2008). Godly play: an intervention for improving physical, emotional, and spiritual responses of chronically ill hospitalized children. The Journal of Pastoral Care and Counseling, 62(3), 261-271.

Feudtner, C., Haney, J., \& Dimmers, M. (2003). Spiritual needs of hospitalized children and their families: A national survey of pastoral care providers' perceptions. Pediatrics, 111(1), e67-e72.

Field, N. P., \& Friedrichs, M. (2004). Continuing bonds in coping with the death of a husband. Death Studies, 28, 597-620.

Field, N. P., Gal-Oz, E., \& Bonanno, G. A. (2003). Continuing bonds and adjustment at 5 years after the death of the spouse. Journal of Consulting and Clinical Psychology, 71, 110- 117.

Field, N. P., Orsini, L., Gavish, R., \& Packman, W. (2009). Role of attachment in response to pet loss. Death Studies, 33, 334-355. 
Fisher, J. W. (2009). Getting the balance. Assessing spirituality and well-being among children and youth. International Journal of Children's Spirituality, 14(3), 27388 .

Fletcher, J., Mailick, M., Song, J., \& Wolfe, B. (2013). A sibling death in the family: Common and consequential. PubMed Central, 50(3), 803-826.

Flor, D. L., \& Knapp, N. F. (2001). Transmission and transaction: Predicting adolescents' internalization of parental religious values. Journal of Family Psychology, 15, 627-645.

Folkman S, \& Greer S. (2000). Promoting psychological well-being in the face of serious illness: When theory, research and practice inform each other. Psychooncology, 9(1), 11-19.

Fontana, A. \& Reid Keene, J. (2009). Death and dying in America. Malden, MA: Polity Press.

Forward, D. R., \& Garlie N. (2003). Search for new meaning: Adolescent bereavement after the sudden death of a sibling. Canadian Journal of School Psychology, $18(1-2), 23-53$.

Foster, T. L., \& Gilmer, M. J. (2008). Continuing bonds: A human response within pediatric palliative care. International Journal of Palliative Care Nursing, 14, 8591 .

Foster, T. L. Gilmer, M. J., Davies, B., Dietrich, M. S., Barrera, M., Fairclough, D. L., Gerhardt, C. A. (2011). Comparison of continuing bonds reported by parents and siblings after a child's death from cancer, 35, 420-440.

Foster, T. L., Gilmer, M. J., Vannatta, K., Barrera, M, Davies, B., Dietrich, M. S., . . Gerhardt, C. A. (2012). Changes in siblings after the death of a child from cancer. Cancer Nursing, 35(5), 347-354.

Fowler, J. W. (1981). Stages offaith: The psychology of human development and the quest for meaning. San Francisco: Harper and Row.

Fowler, J. W., \& Dell, M. L. (2004). Stages of faith and identity: Birth to teens. Child and Adolescent Psychiatric Clinics of North America, 13, 17-33.

Frazier, P., Tashiro, T., \& Berman, M. (2004). Correlates of levels and patterns of positive life changes following sexual assault. Journal of Consulting and Clinical Psychology, 72(1), 19-30. 
Friedman, R. J. \& Butler, L. F. (1979). Development and evaluation of a test battery to assess childhood depression. Ontario Institute for Studies in Education.

Fristad, M. A., Cerel, J., Goldman, M., Weller, E. B., \& Weller, R. A. (2000). The role of ritual in children's bereavement. Omega, 42(4), 321-339.

Gall, T. L., Charbonneau, C., Clarke, N. H., Grant, K., Joseph, A., \& Shouldice, L. (2005). Understanding the nature and role of spirituality in relation to coping and health: A conceptual framework. Canadian Psychology, 46(2), 88-104.

Gamino, L.A., Sewell, K. W., \& Easterling, L. W. (2000). Scott and White grief studyphase 2: Toward an adaptive model of grief. Death Studies, 24, 633-660.

Garssen, B., Uwland-Sikkema, N. F., Visser, A. (2015) How spirituality helps cancer patients with the adjustment to their disease. Journal of Religious Health, 54, 1249-1265.

Geckova, A., Pudelsky, M., \& Tuinstra, J. (2000). Peer contacts, social network, and social support from adolescents' point of view. Psychologia a Patasychologia Dietata, 35,121-136.

Gerhardt, C. A., Fairclough, D. L., Grossenbacher, J. C., Barrera, M., Gilmer, M. J., Foster, T. L., Compas, B. E., Davies, B., Hogan, N. S., \& Vannatta, K. (2012). Peer relationships of bereaved siblings and comparison classmates after a child's death from cancer. Journal of Pediatric Psychology, 37(2), 209-219.

Good, M., Willoughby, T., \& Busseri, M. (2011). Stability and change in adolescent spirituality/religiosity: A person centered approach. Developmental Psychology, 47, 538-550.

Gray, C. M., Carter, R., \& Silverman, W. K. (2011). Anxiety Symptoms in African American Children: Relations with ethnic pride, anxiety sensitivity, and parenting. Journal of Child Family Studies, 20, 205-213.

Gray, L. B., Weller, R. A., Fristad, M., \& Weller, E. B. (2011). Depression in children and adolescents two months after the death of a parent. Journal of Affective Disorders, 135, 277-283.

Grossoehme, D. H. (2008). Development of a spiritual screening tool for children and adolescents. The Journal of Pastoral Care and Counseling, 62(1).

Grossoehme, D. H., Cotton, S., \& Leonard, A. (2007). Spiritual and religious experiences of adolescent psychiatric inpatients vs. healthy peers. The Journal of Pastoral Care and Counseling, 61(3), 197-204. 
Grover, R. L., Ginsburg, G. S., \& Ialongo, N. (2005). Child predictors of anxiety symptoms: A longitudinal study. Child Psychiatry and Human Development, 36(2), 133-153.

Gudmundsdottir, M., Chesla, C. A. (2006). Building a new world. Journal of Family Nursing, 12(2), 143-164.

Guite, J., Lobato, D., Kao, B., \& Plante, W. (2004). Discordance between sibling and parent reports of the impact of chronic illness and disability on siblings. Children's Health Care, 33, 77-92.

Gunnestad, A., Thwala, S. (2011). Resilience and religion in children and youth in southern Africa. International Journal of children's Spirituality, 16(2), 169-185.

Gupta, R., \& Yick, A. G. (2002). Chinese cultural dimensions of death, dying, and bereavement: Focus group findings. Journal of Cultural Diversity, 9(2), 32-47.

Haine, R. A., Ayers, T.S., Sandler, I. N., Wolchik, S. A., \& Weyer, J. L. (2003). Locus of control and self-esteem as stress moderators or stress mediators in parentally bereaved children. Death Studies, 27, 619-640.

Harris, P. L. (2011). Conflicting thoughts about death. Human Development, 54, 160168

Harrison, L., \& Harrington, R. (2001). Adolescent bereavement experiences: Prevalence, association with depressive symptoms, and use of services. Journal of Adolescence, 24, 159-169.

Hart, T. (2005). Spiritual experiences and capacities of children and youth. In E. C. Rhehlkepartain, P. E. King, L. Wagener, and P. L. Benson (Eds.). The Handbook of Spiritual Development in Childhood and Adolescence (pp. 163-178). Thousand Oaks, CA: Sage Publications.

Hay, D. (2003). How should we nurture children’s spiritual life? Spirited Scotland, 4.

Hedayat, K. (2006). When the spirit leaves: Childhood death, grieving, and bereavement in Islam. Pediatric Critical Care, 9(6), 1282-1291.

Heilferty, C. M. (2004). Spiritual development and the dying child: The pediatric nurse practitioner's role. Journal of Pediatric Healthcare, 18, 271-275.

Hogan, N. (1988). The effects of time and adolescent sibling bereavement process. Pediatric Nursing, 14, 333-335. 
Hogan, N. S., \& DeSantis, L. (1996). Basic constructs of a theory of adolescent sibling Bereavement. In D. Klass, P. R. Silverman, \& S. L. Nickman (Eds.), Continuing bonds: New understandings of grief (pp. 235-254). Washington, DC: Taylor \& Francis.

Hogan, N. S., \& DeSantis, M. (1994). Things that help and hinder adolescent sibling bereavement. Western Journal of Nursing Research, 16, 132-153.

Hogan, N. S., \& DeSantis, M. (1992). Adolescent sibling bereavement: An ongoing attachment. Qualitative Health Research, 2, 159-177.

Hogan, N. S. (1988). The effects of time on the adolescent sibling bereavement process. Pediatric Nursing, 14, 333-335.

Holloway, K. (2002). Passed on: American mourning stories. Durham, London: Duke University Press.

Holmbeck, G. N., Bruno, E. F., \& Jandasek, B. (2006). Longitudinal research in pediatric psychology: An introduction to the special issue. Journal of Pediatric Psychology, 31, 995-1001.

Horsley, H., \& Patterson, T. (2006). The effects of a parent guidance intervention on communication among adolescents who have experienced the sudden death of a sibling. American Journal of Family Therapy, 34(2), 119-137.

Houskamp, B. M., Fisher, L. A., \& Stubert, M. L. (2004). Spirituality in children and adolescents: research findings and implications for clinicians and researchers. Child and Adolescent Psychiatric Clinics of North America, 13, 221-230.

Hsu, C., O'Connor, M., \& Lee, S. (2009). Understanding of death and dying for people of Chinese origin. Death Studies, 33, 153-174.

Hufton, E. (2006). Parting gifts: The spiritual needs of children. Journal of Child Health Care, 10(3), 240-250.

Jacobs, S., Mazure, C., \& Prigerson, H. (2000). Diagnostic criteria for traumatic grief. Death Studies, 24(3), 185-199.

Jochim, C. (1986). Chinese religions: A cultural perspective. Englewood Cliffs, NJ: Prentice Hall.

Josephson, A. M., \& Dell, M. L. (2004). Religion and spirituality in child and adolescent psychiatry: A new frontier. Child and Adolescent Psychiatric Clinics of North America, 13, 1-15. 
Kamper, R., Cleve, L. V., \& Savedra, M. (2010). Children with advanced cancer: Responses to a spiritual quality of life interview. Journal for Specialists in Pediatric Nursing, 15(4), 301-306. doi: 10.1111/j.1744-6155.2010.00253.x

Kaplow, J. B., Layne, C. M., \& Pynoos, R. S. (2014). Parental grief facilitation: How parents can help their bereaved children during the holidays. Traumatic Stress Points.

Kaplow, J. B., Layne, C. M., Pynoos, R. S., Cohen J. A., \& Lieberman, A. (2012) DSM$\mathrm{V}$ diagnostic criteria for bereavement-related disorders in children and adolescents: developmental considerations. Psychiatry, 75, 243-266.

Kassis, H. (1997). Islam. In H. Coward (Ed.) Life after death in world religions (pp. 4865). Marynkoll, NY: Orbis Books.

Kaufman, K. N., \& Kaufman, N. D. (2006). And then the dog died. Death Studies, 30, 61-76.

Kazak, A. E., \& Noll, R. B. (2004). Child death from pediatric illness: Conceptual intervention approaches from a family/systems and public health perspective. Professional Psychology, 35(3), 219-226.

Kenny, G. (1999). Assessing children's spirituality: What is the way forward? British Journal of Nursing, 81, 25-33.

Kerns, V. (1997). Women and the ancestors: Black Carib kinship and ritual. Chicago, IL: University of Illinois Press.

King, P. E., Furrow, J. L., \& Roth, N. (2002). The influence of families and peers on adolescent religiousness. Journal of Psychology and Christianity, 21, 109-120.

Klass, D., \& Goss, R. (2003). The politics of grief and continuing bonds with the dead: The cases of Maoist China and Wahhabi Islam. Death Studies, 27, 787-811.

Koenig, H. G. (2003). Healthcare and faith communities: How are they related? Journal of General Internal Medicine, 18, 962-963.

Koenig, H. G. (1995). Research on religion and aging. New York: Greenwood Press.

Koenig, H. G. (2004). Spirituality, wellness quality of life. Sexuality, reproduction, and Menopause, 2(2), 76-82.

Kohlberg, L. (1981). Essays on Moral Development: Vol 1. The philosophy of moral development. New York: Harper \& Row. 
Kovacs, M. (1980). Rating Scales to assess depression in school-aged children. Acta paedopsychiat (46), 305-315.

Kramer, K. P. (1988). The sacred art of dying: How world religions understand death. New York: Paulist Press.

Kreider, R. M. (2007). Living arrangements of children: 2004. Current Population Reports (pp. 70-114). Washington, DC: U.S. Census Bureau.

Krok, F., \& Dariusz, U. (2008). The role of spirituality in coping: Examining the relationships between spiritual dimensions and coping styles. Mental Health, Religion and Culture, 11(7), 643-653.

Latham, A. E., \& Prigerson, H. G. (2004). Suicidality and bereavement: complicated grief as psychiatric disorder presenting greatest risk for suicidality. Suicide Life Threat Behavior, 34, 350-362.

Lazarus, R. S., \& Folkman, S. (1984). Stress, appraisal, and coping. New York: Springer Publishing.

Lee, E. (1991). Mourning rituals in Chinese culture. In E. Walsh and M. McGolrick (Eds.), Living beyond loss: Death in the family (pp. 200-204). New York, NY: W.W. Norton and Company.

Leever, M., Deegan-Krause, B., Leliaert, R. M., Richter, K., \& Nelson, P. (2004). "Baptizing" deceased infants? Health Progress, 85(6), 44-49.

Levin, J., \& Chatters, L. (1998). Research on religion and mental health: An overview of empirical findings and theoretical issues. In H. G. Koenig (Ed.), Handbook of religion and mental health (pp. 33-50). San Diego, CA: Academic Press.

Lewis, N. D. (2009). Apolytrosis as ritual and sacrament: Determining a ritual context for death in second-century Marcosian Valentinianism. Journal of Early Christian Studies, 17(4), 525-561.

Lobar, S. L., Youngblut, J. M., \& Brooten, D. (2006). Cross cultural beliefs, ceremonies, and rituals surrounding death of a loved one. Pediatric Nursing, (32)1, 44-50.

Lohan, J. A., \& Murphy, S. A. (2001-2002). Parents' perceptions of adolescent sibling responses after an adolescent or young adult child's sudden, violent death. Omega, 44, 195-213. 
Loike, J., Gillick, M., Mayer, S., Prager, K., Simon, J. R., Steinberg, A., et al. (2010). The critical role of religion: Caring for the dying patient from an Orthodox Jewish perspective. Journal of Palliative Medicine, 13(10), 1267-1271.

Lyon, M. E., Garvie, P., He, J., Malow, R., McCarter, R., \& D’Angelo, L. J. (2012). Spiritual wellbeing among HIV-infected adolescents and their families, Journal of Religion and Health, 53(3), 637-653.

Mack, K. Y. (2001). Childhood family disruptions and adult well-being: The differential effects of divorce and parental death. Death Studies, 25, 419-443.

Maier, E. H., \& Lachman, M. E. (2000). Consequences of early parental loss and separation for health and wellbeing in midlife. International Journal of Behavioral Development, 24, 183-189.

Marks, N. F., Jun, H., \& Song, J. (2007). Death of parents and adult psychological and physical well-being: A prospective U.S. national study. Journal of Family Issues, 28, 1611-1638.

Martinson, I. M., \& Campos, R. G. (1991). Adolescent bereavement: long-term responses to a sibling's death from cancer. Journal of Adolescent Research, 6, 54-69.

McCree, D. H., Wingwood, G. M., DiClemente, R., Davies, S., \& Harrington, K. F. (2003). Religiosity and risky sexual behavior in African-American adolescent females. Journal of Adolescent Health, 3391), 2-8.

Melhem, N. M., Moritz, G., Walker, M., Shear, K. M., \& Brent, D. (2007). Phenomenology and correlates of complicated grief in children and adolescents. Journal of the American Academy of Child and Adolescent Psychiatry, 46(4), 493-499.

Milan, A., \& Hamm, B. (2003). Across the generations: Grandparents and grandchildren. Canadian Social Trends, Winter(71), 2-7.

Miller, W. R., \& Thorensen, C. E. (1999). Spirituality and health. In W. R. Miller (Ed.), Integrating spirituality into treatment (pp. 161-173). Washington, DC: American Psychological Association.

Mueller, P. S., Plevak, D. J., \& Rummans, T. A. (2001). Religious involvement, spirituality, and medicine: Implications for clinical practice. Mayo Clinic Proceedings, 76, 1125-1235.

Munet-Vilaro, F. (1998). Grieving and death rituals of Latinos. Oncology Nursing, 25, 1761-1763. 
Muris, P., Schmidt, H., \& Merckelbach, H. (2000). Correlations among two self-report questionnaires for measuring DSM - defined anxiety disorder symptoms in children: The screen for child anxiety related emotional disorders and the Spence children's anxiety scale. Personality and Individual Differences, (28), 333-346.

Murphy, S. A. (1991). Parent bereavement survey. Unpublished manuscript, University of Washington, Seattle, Washington.

Nagy, M. (1948). The child's theories concerning death. Journal of Genetic Psychology, 73, 3-27.

Nazarko, L. (2006). As death approaches: Cultural issues. Nursing and Residential Care, $8(10), 441-444$.

Neimeyer, R. A., Baldwin, S. A., \& Gillies, J. (2006). Continuing bonds and reconstructing meaning: Mitigating complications in bereavement. Death Studies, 30, 715-738.

Neimeyer, R. A. \& Hogan, N. S. (2001). Quantitative or qualitative? Measurement issues in the study of grief. In M. S. Stroebe, R. O. Hansson, W. Stroebe, \& H. Schut (Eds.), Handbook of bereavement research (pp. 89118). Washington, DC: American Psychological Association.

Neimeyer, R. A., Wittkowski, J., \& Moser, R. P. (2004). Psychological research on death attitudes: An overview and evaluation. Death Studies, 28, 309-340.

Neuman, M. E. (2011). Addressing children's beliefs through Fowler's stages of Faith. Journal of Pediatric Nursing, 26, 44-50.

Nolbris, M., \& Hellstrom, A. L. (2005). Siblings' needs and issues when a brother or sister dies of cancer. Journal of Pediatric Oncology Nursing, 22(4), 227-233.

O'Gorman, S. M. (1998). Death and dying on contemporary society: An evaluation of current attitudes and the rituals associated with death and dying and their relevance to recent understanding of health and healing. Journal of Advanced Nursing, 27, 1127-1135.

Oltjenbruns, K. A. (2001). Developmental context of childhood: Grief and regrief phenomena. In M. S. Stroebe, R. O. Hansson, W. Stroebe, \& H. Schut, (Eds.), Handbook of bereavement research: Consequences, coping and care (pp. 169197). Washington, DC: American Psychological Association.

Packman, W. Horsley, H., Davies, B., \& Kramer, R. (2006). Sibling bereavement and continuing bonds. Death Studies, 30, 817-841. 
Pallant, J. (2010). Multivariate analysis of variance. SPSS survival manual (p. 290). Berkshire, England: McGraw-Hill.

Pang, T. H. C., \& Lam, C. W. (2002). The widower's bereavement process and death rituals: Hong Kong Experiences. Illness, Crisis, and Loss, 10(4), 294-303.

Pargament, K. I. (2002). The bitter and the sweet: An evaluation of the costs and benefits of religiousness. Psychological Inquiry, 13(3), 168-181.

Pargament, K., Koenig, H. C., \& Perez, L. M. (2000). The many methods of religious coping: Development and initial validation of the RGOPE. Joumal of Clinical Psychology, 56, 519-543.

Pargament, K. I., Koenig, H. G., \& Perez, L. M. (2000). The many methods of religious coping: Development and initial validation of the RCOPE. Journal of Clinical Psychology, 56(4), 519-543.

Pargament, K. I. (2001). Religious struggle as a predictor of mortality among medically ill elderly patients. Archives of Internal Medicine, 161, 1881-1885.

Pargament, K. I. (1997). The psychology of religion and coping. New York: The Guildford Press.

Paris, M. M., Carter, B. L., Day, S. X., \& Armsworth, M. W. (2009). Grief and trauma in children after the death of a sibling. Journal of Child and Adolescent Trauma, 2, 71-80.

Park, C. L., \& Folkman, S. (1997). Meaning in the context of stress and coping. Review of General Psychology, 1(2), 115-144.

Parkes, C. M., Laungani, P. \& Young, B. (1997). Death and bereavement across cultures. London: Routledge.

Parry, J. K., \& Shen Ryan, A. (1995). A cross cultural look at death, dying, and religion. Chicago, IL: Nelson-Hall.

Pearce, M. J., Little, T. D., \& Perez, J. E. (2003). Religiousness and depressive symptoms among adolescents, 32(2), 267-276.

Pendleton, S. M., Cavalli, K. S., Pargament, K. I., \& Nasr S. Z. (2002).

Religious/Spiritual coping in childhood cystic fibrosis: a qualitative study.

Pediatrics, 109(1). doi: 10.1542/peds.109.1.e8. 
Pike, S. (2009). Why prince Charles instead of "Princess Mononoke?": A response to the encyclopedia of religion and nature. Journal of the American Academy of Religion, 77(1), 66-72.

Poznaski, E. O., Grossman, J. A., Buchsbaum, Y., M., B., Freeman, L., \& Gibbons, R. (1984). Preliminary studies of the reliability and validity of the children's depression rating scale. Journal of the American Academy of Child Psychiatry, 23, 191-197.

Prigerson, H. G., Bierhals, A. J., Kasl, S. V. (1997). Traumatic grief as risk factor for mental and physical morbidity. American Journal of Psychiatry, 154, 616-623.

Prigerson, H. G., Bridge, J., \& Maciejewski. (1999). Influence of traumatic grief on suicidal ideation among young adults. American Journal of Psychiatry, 156, 1994-1995.

Prosser, R., Korman, D., \& Feinstein, A. (2012). An Orthodox perspective of the Jewish end-of-the Jewish end-of-life experience. Home Healthcare Nurse, 30(10), 579585.

Purow, B., Alisanski, S., Putnam, G., \& Ruderman, M. (2011). Spirituality and pediatric cancer. Southern Medical Journal, 104(4), 299-302.

Ramey, S. L., Ramey, C. T., Philips, M. M., Lanzi, R. G., Brezausek, C, Katholi, C. R., et al. (2000). Report on the national head start/public school early childhood transition demonstration study. Head Start Children's Entry into Public School. Washington, DC: U.S. Department of Health and Human Services.

Reeves, N. C. (2011). Death acceptance through ritual. Death Studies, 35, 408-419. Regnerus, M. D., Burdett, A. (2006). Religious change and adolescent family dynamics. The Sociological Quarterly, 47, 175-194.

Reich, W., \& Welner, Z. (1988). The diagnostic interview for children and adolescentsRevised-Child/parent forms. St. Louis, MO: Washington University.

Ridgely, S. (2012). Children and Religion. Religion Compass, 6(4), 236-248.

Ronen, R., Packman, W., Field, N. P., Davies, B., Kramer, R., \& Long, J. K. (2009). The relationship between grief adjustment and continuing bonds for parents who have lost a child. Omega: The Journal of Death and Dying, 60, 1-31.

Rosen, H. (1986). When a sibling dies. International Journal of Family Psychiatry, 7, 9396. 
Rostila, M., \& J. M. Saarela. (2011). Time does not heal all wounds: Mortality following the death of a parent. Journal of Marriage and Family, 73, 236-249.

Sable, J. (2009). Caring for older Jews. Nursing and Residential Care, 11(12), 621-623.

Sagara-Rosemeyer, M., \& Davies, B. (2007). The integration of religious traditions in Japanese children's view of death and afterlife. Death Studies, 31, 223-247.

Sandler, I. N., Ma, Y., Tein, J., Ayers, T. S., Wolchik, S., Kennedy, C., \& Millsap, R. (2010). Long-term effects of the family bereavement program on multiple indicators of grief in parentally bereaved children and adolescents. Journal of Consulting and Clinical Psychology, 78(2), 131-143.

Sandler, C. L., Robinson, E., \& Carter, B. S. (2012). Loss in the NICU: Sibling matters. American Journal of Hospice and Palliative Medicine, 30(6), 566-580. 1-3.

Saylor, C. F., Finch, A. J., Spirito, A., Bennett, B. (1984). The Children's Depression Inventory: A systematic evaluation of psychometric properties. Journal of Consulting and Clinical Psychology, 52 (6), 955-967.

Schmiege, S. J., Khoo, S. T., Sandler, I. N., Ayers, T. S., \& Wolchik, S. A. (2006). Symptoms of internalizing and externalizing problems after the death of a parent. American Journal of Preventive Medicine, 31, S152-S160.

Schottenbauer, M. A., Spernak, S. M., \& Hellstrom, I. (2007). Relationship between family religious behaviors and child well-being among third-grade children. Mental Health, Religion, and Culture, 10(92), 191-198.

Schwab, R., \& Petersen, K. U. (1990). Religiousness: Its relationship to loneliness, neuroticism, and subjective wellbeing. Journal for the Scientific Study of Religion, 30(4), 381-394.

Schwartz, K. D. (2006). Transformations in parent and friend faith support predicting adolescents' religious faith. International Journal for the Psychology of Religion, 16(4), 311-326.

Seecharan, G. A., Andresen, E. M., Norris, K., \& Toce, S. S. (2004). Parents' assessment of quality of care and grief following a child's death. Archives of Pediatric and Adolescent Medicine, 158, 515-520.

Servaty-Seib, H., Pistole, M. (2006). Adolescent grief: Relationship category and emotional closeness. Omega, 54, 147-167.

Shaefer, J. (1999). When an infant dies: Cross cultural expression of grief and loss. Washington, DC: US Department of Health and Human Services, Public Health Service. 
Shear, M. K., Simon, N., Wall, M., Zisook, S., Neimeyer, R., \& Duan, N. (2011). Complicated grief and related bereavement-issues for DSM5. Depression Anxiety, 28, 103-117.

Siegel, K. Schrimshaw, E. W. (2002). The perceived benefits of religious and spiritual coping among older adults living with HIV/AIDS. Journal for the Scientific Study of Religion, 41(1), 91-102.

Sifkers, J. S., Warren, S. K., J. S., \& Jackson, Y. (2012). Measuring spirituality in children. Journal of Psychology and Christianity, 31(3), 205-214.

Sirkia, K., Saarinen-Pihkala, U., Hovi, L. (2000). Coping of parents and siblings with the death of a child with cancer: Death after terminal care compared with death during active anticancer therapy. Acta Paediatrica Journal, 89, 717-721.

Slaughter, V., \& Griffiths, M. (2007). Death understanding and fear of death in young children. Clinical Child Psychology and Psychiatry, 12, 525-535.

Sood, A. B., Razdan, A., Weller, E. B., \& Weller, R. A. (2006). Children's reaction to parental and sibling death. Current Psychiatry Reports, 8, 115-120.

Softing, H., Dyregrov, A., \& Dyregrov, K. (2016). Because I'm also part of the family. Children's participation in rituals after the loss of a parent or sibling: A qualitative Study From the children's perspective. Journal of Death and Dying, 73(2), 141-158.

Sparshott, M. (2004). The spirituality of babies: Respecting the religious beliefs of bereaved Parents. Journal of Neonatal Nursing, 10(5), 152-155.

Spence, H. S., Barrett, P. M., \& Turner, C. M. (2003). Psychometric properties of the Spence Children's anxiety scale with young adolescents. Anxiety Disorders (17), 605-625.

Spuij M., Prinzie, P., Zijderlaan, J., Stikkelbroek, Y., Dillen, L., Roos, C., \& Boelen, P. (2012). Psychometric properties of the Dutch inventories of prolonged grief for children and adolescents. Clinical Psychology and Psychotherapy, 19(6), 540551 .

Spuij, M., Reitz, E., Prinzie, P., Stikkelbroek, Y., Roos, C., \& Boelen, P.A. (2012). Distinctiveness of symptoms of prolonged grief, depression, and post-traumatic stress in bereaved children and adolescents. European Child Adolescent Psychiatry, 21, 673-679. 
Stormshak, E. A., Bullock, B. M., \& Falkenstein, C. A. (2009). Harnessing the power of sibling relationships as a tool for optimizing social-emotional development. New Directions for Child and Adolescent Development, 126, 61-77.

Suhail, K., Jamil, N., Oyebode, J., \& Ajmal, M. A. (2011). Continuing bonds in bereaved Pakistani Muslims: Effects of culture and religion. Death Studies, 35, $22-41$.

Takriti, R. A., Barrett, M. D., and Buchanan-Barrow, E. (2006). Children's understanding of religion: Interviews with Arab-Muslim, Asian-Muslim, Christian and Hindu children aged 5-11 years. Mental Health, Religion \& Culture, 9 (1), 29 - 42.

Talik, E. B. (2011). The adolescent religious coping questionnaire. Translation and cultural adaptation of Pargament's RCOPE scale for Polish adolescents. Journal of Religious Health. Doi: 10.1007/s10943-011-9464-x

Tanner, J. G. (1995). Death, dying, and grief in the Chinese-American culture. In J. K. Parry and A. S. Ryan (Eds), A cross cultural look at death, dying, and religion (pp. 183-192). Chicago, IL: Nelson Halls Publishers, United States Census.

Thompson et al. (2011). A qualitative study of advice from bereaved parents and siblings. Journal of Social Work in End of Life and Palliative Care, 7, 153-172.

Tuck, I., Alleyne, R., \& Thinganjana, S. (2006). Spirituality and stress management in healthy adults. Journal of Holistic Nursing, 24, 245-253.

Ungureanu, I., \& Sandberg, J. G. (2010). "Broken together": Spirituality and religion as coping strategies for couples dealing with the death of a child: A literature review with clinical implications. Contemporary Family Therapy, 32, 302-319.

Vasta, R., Miller, S. A., \& Ellis, S. (2004). Child Psychology. Hoboken, NJ: John Wiley $\&$ Sons.

Venhorst, C. (2012). Islamic death rituals in a small town context in the Netherlands: Explorations of a common praxis for professionals. Omega, 65(1), 1-10.

Wahlhaus, E. (2005).The psychological benefits of the traditional Jewish Mourning rituals: Have the Changed instituted by the progressive movement enhanced or diminished them? European Judaism, 38(1), 95-109.

Weeks, O. D. (2004). Comfort and healing: Death ceremonies that work. Illness, Crisis, \& Loss, 12(2), 113-125.

Weller, E. B., \& Weller, R. A. (1979). Diagnostic interview for depression in children and adolescents (DIDCA). Unpublished manuscript, University of Kansas Medical Center, Kansas City, Kansas. 
Weller, E. B., \& Weller, R. A., \& Fristad, M. A. (1984a). The funeral questionnairechild and parent forms. Unpublished manuscript, University of Kansas Medical Center, Kansas City, Kansas.

Weller, E. B., \& Weller, R. A., \& Fristad, M. A. (1984b). The grief inventory-Parent and adolescent forms. Unpublished manuscript, University of Kansas Medical Center, Kansas City, Kansas.

Yang, S. (2012). An autophotography of the young adult children of bereaved families in Korea Journal of Loss and Trauma, 17, 1-11.

Yang, S. C., \& Chen, S. (2006). Content analysis of free-response narratives to personal meanings of death among Chinese children and adolescents, 30(3), 217-241.

Yick, A. G., \& Gupta, R. (2002). Chinese cultural dimensions of death, dying, and bereavement:Focus group findings. Journal of Cultural Diversity, 9(2), 32-43.

Youngblut, J. M., \& Brooten, D. (2013). Parent report of child response to sibling death in a neonatal or pediatric ICU. American Journal of Critical Care, 22(6), 474480.

Walker, D. F., Reid, H. W., O’Neill, T., \& Brown, L. (2009). Changes in personal religion/spirituality during and after childhood abuse: A review and synthesis. Psychological Trauma: Theory, Research, Practice, and Policy, 1, 130-145. doi: 10.1037/a0016211.

Westerink, D., \& Stroebe, M. (2012). The death of a grandparent: Kaey's story. Bereavement Care, 31(1), 6-10.

Wilkins, K. L., \& Woodgate, R. L. (2005). A review of qualitative research on the childhood cancer experience from the perspective of siblings: A need to give them a voice. Journal of Pediatric Oncology Nursing, 22,305-319.

Willis, C. A. (2002). The grieving process in children: Strategies for understanding, educating, and reconciling children's perceptions of death. Early Childhood Education Journal, 29, 221-226.

Wilson, R. A. (2010). The spiritual life of children. Wellness and Nature, 195, 24-27.

Wolchick, S. A., Tein, J. Y., Sandler, I. N., \& Ayers, T. S. (2006). Stressors, quality of the child-caregiver relationship, and children's mental health problems after parental death: The mediating role of the self-systems beliefs. Journal of Abnormal Child Psychology, 34(2), 221-238. 
Wolowelsky, J. B. (1996). Communal and individual mourning dynamics within traditional Jewish law. Death Studies, 20, 460-480.

Woodgate, R. L. (2006). Sibling's experiences with childhood cancer: A different way of being in the family. Cancer Nursing, 29, 406-414.

Woolley, J. D., \& Phelps, K. E. (2000). The development of children's beliefs about prayer. Journal of Cognition and Culture, 1, 139-167.

Woolley, J. D., Phelps, K. E., Davis, D. L., \& Mandell, D. J. (1999). Where theories of mind meet magic. The development of children's beliefs about wishing. Child Development, 70, 571-587.

Wong, Y. J., Rew, L., Slaikeu, K. D. 2006. A systematic review of recent research on adolescent religiosity and spirituality and mental health. Issues in Mental Nursing, 27, 161-183.

Worden, J. W., Davies, B., \& McCown, D. (1999). Comparing parent loss with sibling loss. Death Studies, 23(1), 1-15.

Youngblut, J.M., \& Brooten, D. (2013). Parent report of child response to sibling death in a neonatal or pediatric ICU. American Journal of Critical Care, 13, 474-481. doi: 10.4037/ajcc2013790 PMC3881261.

Yust, K. M. (2003). Toddler spiritual formation and the faith community. International Journal of Children's Spirituality, 8(2), 133-149.

Zehnder, D., Vollrath, M., \& Landolt, M. A. (2006). Prospective study of the effectiveness of coping in pediatric patients. Child Psychiatry and Human Development, 36(3), 351-368. 
To Whom it May Concern:

I have met with Ivette Hidalgo PhD Nursing student from Florida International University and she explained her research study to me. I will be able to assist with the recruitment of bereaved children from 8 to 18 years of age.

Sincerely,



Name in Print

Sr. VP, Patient Experience \& Staff Development, Seasons Hospice \& Palliative Care

Title/Organization

$8 / 18 / 15$

Date 
Re: Ivette Hidalgo, MSN, PNP-BC

PhO Student at Florida International University

To Whom It May Concern:

I have met with Ivette Hidalgo PhD student from Florida International University and she explained to me her research study. I will be able to assist her with the recruitment of children from 6 to 18 years of age.

Sincerely,

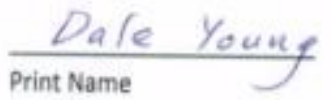



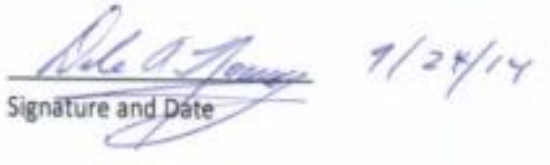




\section{Children's
Bereavement
Center}

Support for children, teens, and aduits after a lass

DiRectons

Mindy Casses Pho

Peter willig LJekt, \&T

Wathy kramer, LCSW, CT August 21, 2014

ExECUTVE ตOAMอ

Nancy Anviey

Eduardo Bertar

wame P Ennner

Alan Erown

Magsie fituon Fode

Sondra Faren.

Julie Fighman Benewat?

Megan Fitrogtrick

Edware Golden

Edwars Doidamen

David Geideneitz

Cuts Turr teunte

Jeftrey Lemmar

tliabosth Leight

Anhiegh Lindenaver

Gary Mansfieid

Abert 'Nox' Mes

Alex Montagoe

Mancon hasasen

Lary Dimpistai

inh sain-mainu

kin M Staplos

Re: Ivette Hidalgo, MSN, PNP-BC

PhD Student at Florida International University

To Whom it May Concern:

I have met with Ivette Hidalgo PhD student from Florida international University and she explained to me her research study as it pertains to the grieving children we serve. I will be able to assist her with the recruitment of children from 6 to 18 years of age that currently attend or have attended the Children's Bereavement Center (CBC) over the past two years.

Please accept this letter as our commitment to assisting with this study.

Sincerely,

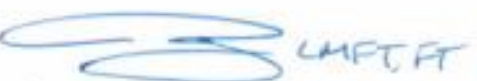

Peter Willig, LMFT, FT

Chief Operating Officer

Children's Bereavement Center 


\section{(1) 'GENTIVA' Hospice}

$9 / 11 / 14$

Re: ivette Hidalgo, MSN, PNP-BC

PhD Student at Florida International University

To Whom it May Concern:

I have met with Ivette Hidalgo PhD student from Florida international University and she explained to me her research study. I will be able to assist her with the recruitment of children from 6 to 18 years of age.

Sincerely,

Jeanna Comendeiro

Print Name

Gentiva Hospice

Title/Organization

Signature and Date

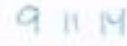

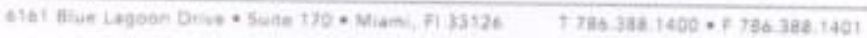






\author{
ADULT CONSENT TO PARTICIPATE IN A RESEARCH STUDY \\ Children's Coping after the Death of a Parent, Grandparent, or Sibling
}

\section{PURPOSE OF THE STUDY}

You are being asked to be in a research study. The purpose of this study is to see how children's and adolescents' use of spiritual coping strategies is different for different ages, genders, race/ethnicity, whether they participated in religious rituals and practices, and whether the deceased was the child's parent, grandparent, or sibling (brother or sister). Another purpose of this study is to see how children's and adolescents' spiritual coping strategies might be related to their grief, personal growth, depression, and anxiety after the death.

\section{NUMBER OF STUDY PARTICIPANTS}

If you participate in this study, you will be one of 120 children and teens in this research study.

\section{DURATION OF THE STUDY}

Your participation will take about 1 hour.

\section{PROCEDURES}

If you participate in this study, we will ask you to do the following things:

1. Tell us about the religious rituals and practices you have participated in, if any.

2. Answer questions about your spiritual coping strategies, grief, anxiety, depression, thoughts about self-harm, and personal growth.

\section{RISKS AND/OR DISCOMFORTS}

The following risks may be associated with your participation in this study: you may become sad when answering the study's questions. Some children and adolescents find that talking to others about their deceased loved one is helpful and makes them feel better. You have the right to skip questions that you do not want to answer.

\section{BENEFITS}

There are no direct benefits for you for being in the study. Researchers and health care providers may benefit from what you tell us in this study in understanding how: 1) children and adolescents use spiritual coping strategies; 2 ) these strategies might affect children's and adolescents' reactions (grief, personal growth, depression, and anxiety) to the death of a parent, grandparent, or sibling: and 3) how health care providers might be able to help children and adolescents when a parent, a grandparent, or a sibling (brother or sister) dies.

\section{ALTERNATIVES}

The alternative is for you not to be in this study. 


\begin{tabular}{|l|l|}
\hline FIU IRB Approval: & $05 / 27 / 2016$ \\
\hline FIU IRB Expiration: & $05 / 27 / 2017$ \\
\hline FIU IRB Number: & IRB-15-0233 \\
\hline
\end{tabular}

\section{CONFIDENTIALITY}

The records of this study will be kept private and will be protected to the fullest extent provided by law. We will not tell your parents about your answers unless we are concerned about your health or safety. You do not have to talk with your parents about your answers.

If we learn about serious harm to you or someone else in your house, we will take steps to protect that person even if it requires telling the authorities without your permission. In these instances, we would only disclose information to the extent necessary to prevent harm.

In any report we might publish, your information will be combined with the information from other children and adolescents. We will not include any information that will make it possible to identify you as being in this study. Research records (the forms with your answers) will be stored securely and only the researcher team will have access to the records. However, your answers may be reviewed for audit purposes by authorized University or other agents who will be bound by the same provisions of confidentiality.

Each family (you and your parent) will be assigned a unique study identification (ID) number that will be placed on every page of the forms. The ID numbers and names will be stored separately from the file with your parent's and your names, phone number, and address. The information you tell us and your signed consent form will be locked in separate drawers in the co-PI's office.

\section{COMPENSATION \& COSTS}

You will receive a $\$ 10$ gift card from Walmart or Target for participating in this study. There are no costs to you to be in this study.

\section{MEDICAL TREATMENT}

Routinely, FIU, its agents, or its employees do not pay for or provide free care for human subjects if they get hurt from being in a research project. If you become ill or injured, contact your regular doctor or health care provider. If you have insurance, your insurance company may or may not pay for these costs. If you do not have insurance, or if your insurance company refuses to pay, you will be billed by your provider. Funds to compensate for pain, expenses, lost wages and other damages caused by injury are not routinely available.

\section{RIGHI TO DECLINE OR WITHDRAW}

Your participation in this study is voluntary. You are free to be in the study or to withdraw your consent at any time during the study. Your withdrawal or lack of participation will not affect any benefits to which you are otherwise entitled. The investigators may remove you from the study without your consent if they feel it is in the best interest of the study.

\section{RESEARCHER CONTACT INFORMATION}

If you have any questions about the purpose, procedures, or any other issues about this research study, you may contact Ivette Hidalgo at Florida International University, 3 
\begin{tabular}{|l|l|}
\hline FIU IRB Approval: & $05 / 27 / 2016$ \\
\hline
\end{tabular}

FIU IRB Expiration: $05 / 27 / 2017$

\begin{tabular}{|l|l|l|}
\hline FIU IRB Number: & IRB-15-0233 \\
\hline
\end{tabular}

\section{IRB CONTACT INFORMATION}

If you would like to talk with someone about your rights of being in this research study or about ethical issues with this research study, you may contact the FIU Office of Research Integrity by phone at 305-348-2494 or by email at oria fiu.edu.

\section{PARTICIPANT AGREEMENT}

I have read the information in this consent form and agree to participate in this study. I have had a chance to ask any questions I have about this study, and they have been answered for me. I understand that I will be given a copy of this form for my records.

Signed Name of Adult Participant

Printed Name of Adult Participant

Signature of Person Obtaining Consent 


 FLORIDA INTERNATIONAL. UNIVERSITY}

\section{CHILD or TEEN ASSENT TO PARTICIPATE IN A RESEARCH STUDY}

Children's Coping after the Death of a Parent, Grandparent or Sibling

\section{WHY ARE YOU DOING THIS STUDY?}

We would like you to be in our research study. A research study is a way to learn information about something. We would like to find out more about how children and teens react to the death of a parent, a grandparent, or a brother or sister and how (or if) they use spiritual and religious practices or things to help them cope with the death. This information may help us to know how to help other children and teens after the death of a parent, a grandparent, or a brother or sister.

HOW MANY OTHERS WILL BE IN THIS STUDY?

If you agree to be in this research study, you will be one of 120 children and teens in the study.

\section{HOW LONG WILL THE STUDY LAST?}

It will take about 1 hour to answer the questions in this study. We will talk with you only this one time.

\section{WHAT WILL HAPPEN IN THIS STUDY?}

If you participate in this study, we will ask you to:

1. Tell us about the religious rituals and practices you have participated in, if any.

2. Answer questions about your spiritual coping strategies, grief, anxiety, depression and personal growth.

CAN ANYTHING BAD HAPPEN TO ME?

You may feel sad when you answer some of our questions. You can skip questions you do not want to answer.

CAN ANYTHING GOOD HAPPEN TO ME?

Sometimes children and teens find that talking to others about the death is helpful and makes them feel better.

\section{DO I HAVE OTHER CHOICES?}

Your only choice is whether or not to take part in this study.

WILL ANYONE KNOW I AM IN THE STUDY?

Your answers are private and will not be shared with your parents. The records of this study will be kept private and will be protected by the researchers. We will only share your answers with your parents if we feel that your safety is in danger. 
WILL I BE GIVEN ANYTHING FOR PARTICIPATING?

You will get a $\$ 10$ gift card from Walmart or Target for being in the study. You will not need to pay for anything to be in this study.

\section{WHAT IF I DO NOT WANT TO DO THIS?}

You do not have to be in this study if you don't want to and you can quit the study at any time. If you don't like a question, you don't have to answer it. No one will get mad at you if you decide you don't want to be in the study.

\section{WHO CAN I TALK TO ABOUT THE STUDY?}

If you have any questions about the research study, you may contact Ivette Hidalgo at Florida International University by phone or text at or by email at or Dr. Youngblut at FIU by phone at or by email at on would like to talk with someone about your rights of being in this research study, you may contact the FIU Office of Research Integrity by phone at 305-348-2494 or by email at ori $a$ fiu.edu.

\section{PARTICIPANT AGREEMIENT}

This research study has been explained to me and I agree to be in this study.

Signature of Child/Teen Participant

Printed Name of Child/Teen Participant

Signafure of Person Obtaining Consent
Date

Page 2 of 2 


\begin{tabular}{|l|l|}
\hline FIU IRB Approval: & $05 / 27 / 2016$ \\
\hline FIU IRB Expiration: & $05 / 27 / 2017$ \\
\hline FIU
\end{tabular}

\section{FIU}

\section{PARENTAL CONSENT TO PARTICIPATE IN A RESEARCH STUDY}

Children's Coping after the Death of a Parent, Grandparent, or Sibling

\section{PURPOSE OF THE STUDY}

You are being asked to be in a research study. You are also being asked to give your permission for your child to be in this research study. The purpose of this study is to see bow children's use of spiritual coping strategies is different for different ages, genders, race/ethnicity, whether they participated in religious rituals and practices, and whether the deceased was the child's parent. grandparent, or sibling (brother or sister). Another purpose of this study is to see how children's spiritual coping strategies might be related to their grief, personal growth, depression, and anxiety after the death.

\section{NUMBER OF STUDY PARTICIPANTS}

If you and your child participate in this study, you will be one of 120 families in this research study.

\section{DURATION OF THE STUDY}

Your participation will take about 15-20 minutes and your child's participation will take about 1 hour.

\section{PROCEDURES}

If you and your child participate in this study, we will ask you to do the following things:

1. Complete an information sheet about your age, education, race/ethnicity, religion, marital or partner status. annual income, number of surviving children in the family and their ages, and about the deceased's age, sex, where be/she died, canse of death, date of death, relationship to child, and whether the child had lived with the deceased.

2. Answer questions about the religious rituals and practices your child bas participated in, if any.

We will also ask your child to do the following thing:

1. Tell us about the religious rituals and practices they have participated in, if any.

2. Answer questions about their spiritual coping strategies, grief, anxiety, depression, thoughts about self-harm, and personal growth.

\section{RISKS AND/OR DISCOMFORTS}

The following risks may be associated with your and your child's participation in this study: you and/or your child may become sad when answering the study's questions. Some adults and children find that talking to others about their deceased loved one is helpful and makes them feel better. You and your child have the right to skip questions that you do not want to answer.

Page 1 of 3 


\begin{tabular}{|l|l|}
\hline FUU IRB Approval: & $05 / 27 / 2016$ \\
\hline FU IRB Expiration: & $05 / 27 / 2017$ \\
\hline FUU IRB Number: & IRA-15-0233 \\
\hline
\end{tabular}

\section{BENEFITS}

There are no direct benefits for you or your child for being in the study. Researchers and health care providers may benefit from what you and your child tell us in this study in understanding how

1) children use spiritual coping strategies 2) these strategies might affect children's reactions (grief, personal growth, depression, and anxiety) to the death of a parent, grandparent, or sibling. and 3) how health care providers might be able to help children when a parent, a grandpareut, or a sibling (brother or sister) dies.

\section{ALTERNATIVES}

The alternative is for you and your child not to be in this study.

\section{CONFIDENTLALITY}

The records of this study will be kept private and will be protected to the fullest extent provided by law. We will not tell you about your child's answers unless we are concerned about your child's health or safety. We also tell the children that they do not have to talk with their parents about their answers.

If we learn about serious harm to you, your child(ren), or someone else in your house, we will take steps to protect that person even if it requires telling the authorities without your permission. If we have reason to believe that your child is being abused, we will report this to the Florida Abuse hotline. In these instances, we would only disclose information to the extent necessary to prevent harm.

In any report we might publish. you and your child's information will be combined with the information from other parents guardians and children. We will not include any information that will make it possible to identify you or your child as being in this study. Research records (the forms with your and your chitd's answers) will be stored securely and only the researcher tean will have access to the records. However, your and your child's answers may be reviewed for audit purposes by authorized University or other agents who will be bound by the same provisions of confidentiality.

Each family (you and your child) will be assigned a unique study identification (ID) number that will be placed on every page of the forms. The ID numbers and names will be stored separately from the file with your and your child's names, phone number, and address. This file, the information you and your child tell us, and your signed consent form and your child's signed assent form will be locked in separate drawers in the co-PI's office

\section{COMPENSATION \& COSTS}

Your child will receive a $\$ 10$ gift card from Walmart or Target for participating in this study. There are no costs to you and your child to be in this study.

\section{MEDICAL TREATMENT}

Routinely, FIU, its agents, or its employees do not pay for or provide free care for human subjects if they get hurt from being in a research project. If you or you child becomes ill or

Page 2 of 3 


\begin{tabular}{|l|l|}
\hline FIU IRB Approval: & $05 / 27 / 2016$ \\
\hline FIU IRB Explration: & $05 / 27 / 2017$ \\
\hline FIU IRB Number: & IRB-15-0233 \\
\hline
\end{tabular}

injured, contact your regular doctor or health care provider. If you have insurance, your insurance company may or may not pay for these costs. If you do not have insurance, or if your insurance company refuses to pay, you will be billed by your provider. Funds to compensate for pain, expenses, lost wages and other damages caused by injury are not routinely available

\section{RIGHT TO DECLINE OR WITHDRAW}

Your participation and your child's participation in this study is voluntary. You and your child are free to be in the study or to withdraw your consent at any time during the study. Your and your child's withdrawal or lack of participation will not affect any benefits to which you are otherwise entitled. The investigators may remove you and your child from the study without your consent if they feel it is in the best interest of the study.

\section{RESEARCHER CONTACT INFORMLATION}

If you have any questions about the purpose, procedures, or any other issues about this research study, you may contact Ivette Hidalgo at Florida International University,

or

\section{IRB CONTACT INFORMATION}

If you would like to talk with someone about your rights or your child's rights of being in this research study or about ethical issues with this research study, you may contact the FIU Office of Research Integrity by phone at $305-348-2494$ or by email at ori $a$ fu.edu.

\section{PARTICIPANT AGREEMENT}

I have read the information in this consent form and agree to participate and to allow my child to participate in this study. I have had a chance to ask any questions I have about this study, and they have been answered for me. I understand that I will be given a copy of this form for my records.

Printed Name of Parent/ Guardian

Printed Name of Child Participant 


\section{How do Children react to the Death of a Family Member?}

We are doing a Research Study to find out.

\section{To be in the study:}

- Parent speaks English or Spanish

- Child, 8 to 18 years old, and speaks English

- Death of a brother or sister, a parent, or a grandparent in the past 2 years

- Parent and child lived together before the death if the deceased was a parent

- Parent and child able to answer questions about themselves and their family

The purpose of this study is to learn how children:

1) React to the death of a brother or sister, a parent, or a grandparent

2) Use religious or spiritual practices around the death and afterwards

To be in this study or to learn more about it: please call, text, or email me. 
Nicole Wertheim College of Nursing \& Health Sciences

FLORIDA INTERNATIONAL UNIVERSITY

\section{¿Cómo reaccionan los niños} después de la muerte de un ser querido?

Estamos haciendo un estudio de investigación para averiguarlo.

Para participar en el estudio:

- El Padre o Madre debe hablar inglés o español

- El Niño(a), debe tener entre 8 y 18 años y debe hablar ingles

- Muerte de un hermano(a), padre o madre, o abuelo(a) en los últimos 2 años

- Si el fallecido era un padre o madre él o ella tienen que haber vivido con el niño(a) antes de morir

- El padre y el(la) niño(a) puedan contestar preguntas sobre ellos mismos y su familia

El propósito de este estudio es aprender como los niños:

1) Reaccionan después de la muerte de un hermano(a), padre o madre, o abuelo(a)

2) Uso de las prácticas religiosas o espirituales durante la muerte y después 
11 Nicole Wertheim College of Nursing \& Health Sciences - Florida INTERnational. University

Para participar en este estudio o para saber más sobre el: por favor llamar o mandar un mensaje de texto, o contactarme a través de correo electrónico.

Ivette Hidalgo, MSN, ARNP

Estudiante de Doctorado en Enfermería en

FIU

Lano: 
My name is Ivette Hidalgo. I am a pediatric nurse practitioner. I am also working on getting my $\mathrm{PhD}$ in Nursing at Florida International University (FIU). I have been working as a pediatric nurse practitioner in the pulmonology office at Nicklaus Children's Hospital (formerly known as Miami Children's Hospital) for the past 12 years. The patients I see are children with chronic and complex medical conditions that are dependent on ventilators (breathing machines), oxygen, and tracheostomies to live. Working with these children and seeing many of them die from their condition has led me to wonder about the spiritual and religious changes or coping of children who have had a parent, grandparent, or sibling die.

I am doing a research study to talk with children about their experiences and feelings in the first 2 years after the death of a brother or sister, a parent, or a grandparent. This study is part of my PhD program at FIU. Dr. Rosa Roche will be helping with the study. She is also a pediatric nurse practitioner in the pulmonology office at Nicklaus Children's Hospital.

The purpose of this study is to learn about children's reactions to the death of a brother or sister, a parent, or a grandparent in the first 2 years after the death. We also want to learn about children's use of religious or spiritual practices around the time of the death and afterwards.

We will ask the parent(s) to answer some questions about themselves and their family, like their age, education, race/ethnicity, and employment, and about the ages and sex of their other children in the family. We will also ask about religious and spiritual practices in your religion and/or culture that are often done around the time of someone's death.

We will ask the children questions about their health and their religious or spiritual practices.

We are looking for families that meet these conditions:

- Death of a brother or sister, parent, or grandparent in the past two years

- Parent who speaks English or Spanish and is older than 18

- Surviving child is 8 to 18 years old and speaks English

- Parent and child lived together when the death happened if the deceased was a parent If you are interested in being in this study or want to learn more about the study, please call, text, or email me.

Thank you,

Ivette Hidalgo, MSN, ARNP

$\mathrm{PhD}$ in Nursing Student at FIU

Cell:

\section{Email:}


VITA

\section{IVETTE M. HIDALGO}

1994-1996

Associate of Arts

University of Florida

Gainesville, Florida

1996-2000

Associate in Science

Miami Dade Community College

Miami, Florida

2000-2003

Registered Nurse

Nicklaus Children's Hospital

Miami, Florida

2001-2002

Bachelors of Science in Nursing

Florida International University

Miami, Florida

2003-2005

Masters of Science in Nursing

Florida International University

Miami, Florida

2005-present

Pediatric Nurse Practitioner

Nicklaus Children's Hospital

Miami, Florida

\section{PUBLICATIONS AND PRESENTATIONS}

Hannan, J., Brooten, D., Youngblut, JM., Hidalgo, I., Roche, R., \& Seagrave, L. (2015). Physical activity and stress in adult Hispanics. Journal of the American Association of Nurse Practitioners, 27(2), 79-86.

Brooten, D., Youngblut, J., Charles, D., Roche, R., Hidalgo, I., Malkawi, F. (2015).

Death rituals reported by White, Black, and Hispanic parents following the ICU death of an infant or child (2016). Journal of Pediatric Nursing, 31, 132-140.

Brooten, D., Youngblut, J., Seagrave, L., Caicedo, C., Hawthorne, D., Hidalgo, I., Roche, R. (2012). Parent's perceptions of health care providers actions around child ICU death: What helped, what did not. American Journal of Hospice \& Palliative Medicine, 30(1), 40-49. 
Ivette Hidalgo PhD Student, Jean Hannan, Dorothy Brooten, JoAnne Youngblut, Rosa Roche PhD Student. (2012, September). Physical activity in adult Latinas. Paper presented at the XIII Pan American Nursing Research Colloquium, Miami. 